\title{
Unitary Boundary Pairs for Isometric Operators in Pontryagin Spaces and Generalized Coresolvents
}

\author{
D. Baidiuk ${ }^{1} \cdot$ V. Derkach ${ }^{2,3}$ (D) S. Hassi ${ }^{4}$
}

Received: 13 July 2020 / Accepted: 12 December 2020 / Published online: 1 February 2021

(c) The Author(s) 2021

\begin{abstract}
An isometric operator $V$ in a Pontryagin space $\mathfrak{H}$ is called standard, if its domain and the range are nondegenerate subspaces in $\mathfrak{H}$. A description of coresolvents for standard isometric operators is known and basic underlying concepts that appear in the literature are unitary colligations and characteristic functions. In the present paper generalized coresolvents of non-standard Pontryagin space isometric operators are described. The methods used in this paper rely on a new general notion of boundary pairs introduced for isometric operators in a Pontryagin space setting. Even in the Hilbert space case this notion generalizes the earlier concept of boundary triples for isometric operators and offers an alternative approach to study operator valued Schur functions without any additional invertibility requirements appearing in the ordinary boundary triple approach.
\end{abstract}

Dedicated to our friend and colleague Henk de Snoo on the occasion of his 75th birthday.

Communicated by Jussi Behrndt.

This article is part of the topical collection "Recent Developments in Operator Theory - Contributions in Honor of H.S.V. de Snoo" edited by Jussi Behrndt and Seppo Hassi.

$凶$ V. Derkach

volodymyr.derkach@tu-ilmenau.de

D. Baidiuk

baydyuk@gmail.com

S. Hassi

sha@uwasa.fi

1 Department of Mathematics, Tampere University, Tampere, Finland

2 Department of Mathematics and Natural Sciences, TU Ilmenau, Ilmenau, Germany

3 Department of Mathematics, Vasyl Stus Donetsk National University, Vinnytsya, Ukraine

4 Department of Mathematics and Statistics, University of Vaasa, Vaasa, Finland

Birkhäuser 
Keywords Pontryagin space - Isometric operator - Boundary triple · Boundary pair · Weyl function · Characteristic function · Generalized coresolvent

Mathematics Subject Classification 47A20 • 47A56 - 47B50 - 46C20

\section{Introduction}

Extension theory for standard symmetric and isometric operators in Pontryagin spaces was first developed by Iokhvidov and Kreĭn in [30], generalized resolvents of such operators were described by Kreı̆n and Langer in [37-39]. Following [28] we will use the notion standard for an isometric operator $V$ in a Pontryagin space $\mathfrak{H}$, if its domain dom $V$ and the range ran $V$ are nondegenerate subspaces in $\mathfrak{H}$. In this case every unitary extension of $V$ can be obtained in pretty much the same way as in the case of Hilbert space isometric operator. Similarly, the extension theory and the theory of generalized coresolvents of standard isometric operators in Kreŭn spaces was built by Dijksma et al. in [28]. For a nonstandard isometric operator in a Pontryagin space, description of its regular (resp. nonregular) generalized coresolvents in Pontryagin spaces without growth (resp. with growth) of negative index was given by Sorjonen [46] (resp. by Nitz $[43,44]$ ). However, the proof in [44] is not so convincing, as it becomes quite complicated and contains some gaps.

Another approach to the extension theory of symmetric operators in Hilbert spaces is based on the concept of abstract boundary value introduced by Calkin [13] and later formalized in the notion of boundary value space in $[33,35]$ (or ordinary boundary triple in [26]). In [25] with each boundary triple there was associated an analytic object-abstract Weyl function which allows to carry out spectral analysis of extensions of symmetric operators. In the case of a Hilbert space isometric operator (and more generally for a dual pair of operators) the notions of a boundary triple and a corresponding Weyl function were introduced in $[41,42]$. These notions, when generalized to the indefinite case in [5], proved to be an adequate language in the extension theory of nonstandard isometric operator $V$ in a Pontryagin space, since they allowed to give full description of generalized coresolvents of $V$. However, the method proposed in [5] is restricted to the case of regular generalized coresolvents, which have minimal realizations in Pontryagin spaces $\widetilde{\mathfrak{H}}$ with the same negative index as $\mathfrak{H}$, and does not work for generalized coresolvents of $V$ which have minimal realizations in Pontryagin spaces $\mathfrak{H}$ with bigger negative indices.

This difficulty can be prevented by using an appropriate notion of boundary pairs, which extend the concept of ordinary boundary triples. In the case of symmetric operators in Hilbert spaces an extension of ordinary boundary triples, a so-called generalized boundary triple, was introduced and studied in [26]. This notion was further generalized in [19] to the notion of a unitary boundary pair (called therein as a boundary relation), which can be applied to study generalized resolvents of symmetric operators $[20,21]$ and various general classes of boundary value problems for ordinary and partial differential operators, see [21-24]. In particular, in [19] it was shown that every Nevanlinna pair (or Nevanlinna family of holomorphic relations) can be realized as the Weyl family of some unitary boundary pair, and in $[18,20]$ this notion was used 
to get a new proof of Kreı̆n formula for generalized resolvents of symmetric operators via the coupling method developed therein. In [8] the notion of unitary boundary pair was introduced for symmetric operators in Pontryagin spaces and it was shown that every generalized Nevanlinna pair, allowing a finite negative index for the associated Nevanlinna kernel, can be realized as the Weyl family of such a unitary boundary pair.

In this paper a new notion of a unitary boundary pair with an associated Weyl function is introduced and studied in the setting of isometric operators in Pontryagin spaces. In particular, it is shown in Sect. 3 how a certain subclass of unitary boundary pairs is connected to unitary colligations (see [1]) and, moreover, that the Weyl functions associated with that subclass of unitary boundary pairs actually coincide with characteristic functions of the corresponding unitary colligations; see Theorems 3.10, 3.11. Furthermore, using some transformation results, being motivated by [1], it is also shown that every operator valued generalized Schur function (not necessarily holomorphic at the origin) can still be realized as the Weyl function of some unitary boundary pair for an isometric operator $V$ in a Pontryagin space; see Theorems 3.15, 3.17. These two theorems show that the present notion of a unitary boundary pair for isometric operators in a Pontryagin (as well as in the classical Hilbert) space setting is a natural object to realize and study generalized (or standard) Schur functions as their Weyl functions. In particular, these new notions complement and extend the approach, which relies on characteristic functions of unitary colligations being associated with the special case stated in Theorem 3.11.

After these characteristic results on unitary boundary pairs and their Weyl function for isometric operators we study in Sect. 4 some spectral properties of proper extensions of $V$ and find a formula for their canonical coresolvents, see Theorem 4.2, and then with these preparations prove an analog of Kreĭn formula for the generalized coresolvents of the isometric operator $V$. This latter problem is solved via the coupling method, where we consider a coupled unitary boundary pair as a direct sum of an ordinary boundary triple and a unitary boundary pair and then derive the formula for generalized coresolvents from the formula for canonical coresolvents associated with the coupled boundary pair.

\section{Preliminaries}

\subsection{Indefinite Inner Product Spaces}

A linear space $\mathfrak{H}$ endowed with an inner product $[\cdot, \cdot]_{\mathfrak{H}}$ is called an inner product space, see [4,11]. A vector $f \in \mathfrak{H}$ is called positive (resp. negative or neutral), if $[f, f]_{\mathfrak{H}}>0$ (resp. $[f, f]_{\mathfrak{H}}<0$ or $[f, f]_{\mathfrak{H}}=0$ ). A subspace $\mathfrak{L} \subset \mathfrak{H}$ is called positive (resp. negative or neutral), if every vector $f \in \mathfrak{L} \backslash\{0\}$ is positive (resp. negative or neutral). The orthogonal complement of a subspace $\mathfrak{L}$ is denoted by $\mathfrak{L}^{[\perp]}$.

An inner product space $\mathfrak{H}$ is called a Kreln space, if it admits a fundamental decomposition

$$
\mathfrak{H}=\mathfrak{H}_{+}[+] \mathfrak{H}_{-}
$$


as an orthogonal sum of a positive subspace $\mathfrak{H}_{+}$and a negative subspace $\mathfrak{H}_{-}$. The operator $J=P_{+}-P_{-}$, where $P_{ \pm}$are orthogonal projections in $\mathfrak{H}$ onto $\mathfrak{H}_{ \pm}$, is called the fundamental symmetry of $\mathfrak{H}$. We will use the notation $(\mathfrak{H}, J)$ for the Krel̆n space $\mathfrak{H}$ with the fundamental symmetry $J$. A Kreĭn space $(\mathfrak{H}, J)$ with a finite negative index $\kappa_{-}(\mathfrak{H}):=\operatorname{dim} \mathfrak{H}_{-}$is called a Pontryagin space.

Every closed subspace $\mathfrak{D}$ of a Pontryagin space $(\mathfrak{H}, J)$ admits the following decomposition

$$
\mathfrak{D}=\mathfrak{D}_{0} \dot{+} \mathfrak{D}_{+} \dot{+} \mathfrak{D}_{-},
$$

where $\mathfrak{D}_{0}=\mathfrak{D} \cap \mathfrak{D}^{[\perp]}$ is a neutral subspace (the isotropic part of $\mathfrak{D}$ ) and $\mathfrak{D}_{+}$and $\mathfrak{D}_{-}$ are closed (uniformly) positive and negative subspaces of $(\mathfrak{H}, J)$; see e.g. [11, Theorem IX.2.5]. We will need the following slightly modified version of this statement.

Lemma 2.1 Every linear subspace $\mathfrak{T}$ of a Pontryagin space $(\mathfrak{H}, J)$ admits the following decomposition

$$
\mathfrak{T}=\mathfrak{T}_{+} \dot{+} \mathfrak{T}_{1},
$$

where $\mathfrak{T}_{+}$is a positive subspace of $(\mathfrak{H}, J)$, such that $\overline{\mathfrak{T}_{+}}$is a maximal positive subspace of $\overline{\mathfrak{T}}$, and $\mathfrak{T}_{1}$ is a $k$-dimensional subspace of $(\mathfrak{H}, J)$, where $k=\operatorname{dim} \overline{\mathfrak{T}} / \overline{\mathfrak{T}_{+}}$.

Proof Let $\mathfrak{D}$ be the closure of $\mathfrak{T}$ in $\mathfrak{H}$ and decompose $\mathfrak{D}$ as in (2.2),

$$
\mathfrak{D}=\mathfrak{D}_{0} \dot{+} \mathfrak{D}_{-} \dot{+} \mathfrak{D}_{+},
$$

where $\mathfrak{D}_{0}=\mathfrak{D} \cap \mathfrak{D}^{[\perp]}, \mathfrak{D}_{-}$, and $\mathfrak{D}_{+}$are closed neutral, negative, and positive subspaces of $(\mathfrak{H}, J)$, respectively. Since $\mathfrak{T}$ is a dense subset of $\mathfrak{D}$ and the subspaces $\mathfrak{D}_{0}$ and $\mathfrak{D}_{-}$are finite dimensional, $\mathfrak{T}$ has a dense intersection with $\mathfrak{D}_{+}$,

$$
\overline{\mathfrak{T} \cap \mathfrak{D}_{+}}=\mathfrak{D}_{+} ;
$$

see e.g. [32, Lemma 2.1]. Denote $\mathfrak{T}_{+}:=\mathfrak{T} \cap \mathfrak{D}_{+}$and let $k=\operatorname{dim}\left(\mathfrak{D}_{0} \dot{+} \mathfrak{D}_{-}\right)$. Since $\overline{\mathfrak{T}}=\mathfrak{D}$ one concludes that there exists a $k$-dimensional subspace $\mathfrak{T}_{1} \subset \mathfrak{T} \backslash \mathfrak{D}_{+}$. The closed subspace $\mathfrak{T}_{1} \subset \mathfrak{T}$ decomposes $\mathfrak{T}$ and (2.4) together with a dimension argument leads to

$$
\overline{\mathfrak{T}_{+} \dot{+} \mathfrak{T}_{1}}=\overline{\mathfrak{T}_{+}} \dot{+} \mathfrak{T}_{1}=\mathfrak{D}_{+} \dot{+} \mathfrak{T}_{1}=\mathfrak{D}=\overline{\mathfrak{T}}
$$

The equality $\overline{\mathfrak{T}}=\mathfrak{T}_{1} \dot{+} \overline{\mathfrak{T}_{+}}$combined with (2.4) yields the decomposition (2.3) for $\mathfrak{T}$.

\subsection{Linear Relations in Krěn Spaces}

Let $\left(\mathfrak{H}_{1}, J_{\mathfrak{H}_{1}}\right)$ and $\left(\mathfrak{H}_{2}, J_{\mathfrak{H}_{2}}\right)$ be two Krel̆n spaces. A linear relation $T$ from $\mathfrak{H}_{1}$ to $\mathfrak{H}_{2}$ is a linear subspace of $\mathfrak{H}_{1} \times \mathfrak{H}_{2}$, see e.g. [2]. Often a linear operator $T: \mathfrak{H}_{1} \rightarrow \mathfrak{H}_{2}$ 
will be identified with its graph

$$
\operatorname{gr} T:=\{\{f, T f\}: f \in \operatorname{dom} T\}
$$

For a linear relation $T$ from $\mathfrak{H}_{1}$ to $\mathfrak{H}_{2}$ the symbols dom $T, \operatorname{ker} T, \operatorname{ran} T$, and mul $T$ stand for the domain, kernel, range, and multivalued part, respectively. The inverse $T^{-1}$ is a relation from $\mathfrak{H}_{2}$ to $\mathfrak{H}_{1}$ defined by $\left\{\left\{f^{\prime}, f\right\}:\left\{f, f^{\prime}\right\} \in T\right\}$. Denote by $\rho(T)$ the resolvent set of $T$, by $\sigma(T)$ the spectrum of $T$ and by $\sigma_{p}(T)\left(\operatorname{resp} . \sigma_{c}(T), \sigma_{r}(T)\right)$ the point (resp. continuous, residual) spectrum of $T$. The adjoint $T^{[*]}$ is the closed linear relation from $\mathfrak{H}_{2}$ to $\mathfrak{H}_{1}$ defined by (see [9])

$$
T^{[*]}=\left\{\{h, k\} \in \mathfrak{H}_{2} \times \mathfrak{H}_{1}:[k, f]_{\mathfrak{H}_{1}}=[h, g]_{\mathfrak{H}_{2}},\{f, g\} \in T\right\} .
$$

The following equalities are obvious from (2.5)

$$
(\operatorname{dom} T)^{[\perp]}=\operatorname{mul} T^{[*]}, \quad(\operatorname{ran} T)^{[\perp]}=\operatorname{ker} T^{[*]} .
$$

A linear relation $T$ from $\mathfrak{H}_{1}$ to $\mathfrak{H}_{2}$ is called isometric (resp. contractive or expanding), if for every $\{f, g\} \in T$ one has

$$
[g, g]_{\mathfrak{H}_{2}}=[f, f]_{\mathfrak{H}_{1}} \quad\left(\text { resp. }[g, g]_{\mathfrak{H}_{2}} \leq[f, f]_{\mathfrak{H}_{1}} \text { or }[g, g]_{\mathfrak{H}_{2}} \geq[f, f]_{\mathfrak{H}_{1}}\right) .
$$

It follows from (2.5) and (2.7) that $T$ is isometric, if $T^{-1} \subseteq T^{[*]}$. A linear relation $T$ from $\mathfrak{H}_{1}$ to $\mathfrak{H}_{2}$ is called unitary, if $T^{-1}=T^{[*]}$, [45]. Moreover, $T$ is said to be a standard unitary operator if $\operatorname{dom} T=\mathfrak{H}_{1}$ and $\operatorname{ran} T=\mathfrak{H}_{2}$. For an isometric linear relation $T$ one obtains from $T^{-1} \subseteq T^{[*]}$ and the identities (2.6) that

$$
\operatorname{ker} T \subseteq(\operatorname{dom} T)^{[\perp]}, \quad \operatorname{mul} T \subseteq(\operatorname{ran} T)^{[\perp]}
$$

For a unitary linear relation the following statements hold, see [45, Theorem 2].

Proposition 2.2 Let $T$ be a unitary relation from the Kre $\breve{n}$ space $\left(\mathfrak{H}_{1}, J_{\mathfrak{H}_{1}}\right)$ to the Kre ̌n space $\left(\mathfrak{H}_{2}, J_{\mathfrak{H}_{2}}\right)$. Then:

(i) dom $T$ is closed if and only if $\operatorname{ran} T$ is closed;

(ii) the following equalities hold:

$$
\operatorname{ker} T=(\operatorname{dom} T)^{[\perp]}, \quad \operatorname{mul} T=(\operatorname{ran} T)^{[\perp]} .
$$

Denote

$$
\mathbb{D}=\{\lambda \in \mathbb{C}:|\lambda|<1\}, \quad \mathbb{D}_{e}=\{\lambda \in \mathbb{C}:|\lambda|>1\}, \quad \mathbb{T}=\{\lambda \in \mathbb{C}:|\lambda|=1\} .
$$

If $V$ is a single-valued closed isometric operator in a Pontryagin space $\mathfrak{H}$ then the subspaces $\operatorname{ran}(V-\lambda I)$ are closed for every $\lambda \in \mathbb{D} \cup \mathbb{D}_{e}$, see e.g. [40, Section 1.3] 
or Lemma 2.4 below, and each of the sets $\sigma_{p}(V) \cap \mathbb{D}$ and $\sigma_{p}(V) \cap \mathbb{D}_{e}$ consist of at most $\kappa=\kappa_{-}(\mathfrak{H})$ eigenvalues, see e.g. [31, p. 49 Corollary 2]. Denote by $\mathfrak{N}_{\lambda}$ the defect subspace of $V$ :

$$
\mathfrak{N}_{\lambda}:=\mathfrak{H}[-] \operatorname{ran}(I-\bar{\lambda} V), \quad \lambda \in \mathbb{D} \cup \mathbb{D}_{e}
$$

Then

$$
\mathfrak{N}_{\lambda}=\operatorname{ker}\left(V^{-[*]}-\lambda I\right)=\left\{f_{\lambda}:\left(f_{\lambda}, \lambda f_{\lambda}\right)^{T} \in V^{-[*]}\right\}
$$

As is known, see [31, Theorem 6.1], the numbers $\operatorname{dim} \mathfrak{N}_{\lambda}$ take a constant value $n_{+}(V)$ for all $\lambda \in \mathbb{D} \backslash{\overline{\sigma_{p}(V)}}^{-1}$, and $n_{-}(V)$ for all $\lambda \in \mathbb{D}_{e} \backslash{\overline{\sigma_{p}(V)}}^{-1}$. The numbers $n_{ \pm}(V)$ are called the defect numbers of $V$.

Definition 2.3 The isometric operator $V$ in $\mathfrak{H}$ is called simple, if $\sigma_{p}(V) \backslash \mathbb{T}=\varnothing$ and

$$
\overline{\operatorname{span}}\left\{\mathfrak{N}_{\lambda}: \lambda \in \mathbb{D} \cup \mathbb{D}_{e}\right\}=\mathfrak{H} .
$$

In the case of Pontryagin spaces some further results on isometric and unitary relations can be established. For any isometric relation $T$ between two Krel̆n spaces it is clear that $\operatorname{ker} T$ and mul $T$ are neutral subspaces. Therefore, in a Pontryagin space ker $T$ and mul $T$ are necessarily finite dimensional. If $T$ is closed then $\operatorname{ker} T$ and mul $T$ are also closed. In Pontryagin spaces the following stronger result is true.

Lemma 2.4 Let $T$ be a closed isometric relation from the Pontryagin space $\left(\mathfrak{H}_{1}, J_{\mathfrak{H}_{1}}\right)$ to the Pontryagin space $\left(\mathfrak{H}_{2}, J_{\mathfrak{H}_{2}}\right)$. Then the domain and the range of $T$ are closed.

Proof The isometry of $T$ means that $T^{-1} \subseteq T^{[*]}$. Taking inverses one gets $T=$ $\left(T^{-1}\right)^{-1} \subseteq T^{-[*]}$, i.e., $T$ and $T^{-1}$ are simultaneously isometric. Therefore, to prove the statement it suffices to prove that the range of $T$ is a closed subspace in $\mathfrak{H}_{2}$, since $T$ is closed precisely when its inverse $T^{-1}$ is closed and clearly dom $T=\operatorname{ran} T^{-1}$.

Now let dom $T$ be decomposed as in Lemma 2.1,

$$
\operatorname{dom} T=\mathfrak{T}_{+} \dot{+} \mathfrak{T}_{1}
$$

so that $\mathfrak{D}_{+}:=\overline{\mathfrak{T}_{+}}$is a maximal uniformly positive subspace of $\mathfrak{D}:=\overline{\operatorname{dom}} T$. Next introduce the restriction of (the graph of) $T$ by setting

$$
T_{+}:=T \cap\left(\mathfrak{D}_{+} \times \mathfrak{H}_{2}\right) .
$$

Then $T_{+}$is closed and as a restriction of $T$ it is also an isometric relation from $\left(\mathfrak{H}_{1}, J_{\mathfrak{H}_{1}}\right)$ to $\left(\mathfrak{H}_{2}, J_{\mathfrak{H}_{2}}\right)$. Moreover, dom $T_{+}=\mathfrak{T}_{+} \subseteq \mathfrak{D}_{+}$is a uniformly positive subspace. This implies that for all $\left\{f, f^{\prime}\right\} \in T_{+}$and some $\delta>0$,

$$
\left[f^{\prime}, f^{\prime}\right]_{2}=[f, f]_{1} \geq \delta\|f\|_{1}^{2},
$$


which shows that ker $T_{+}=\{0\}$, so that $\left(T_{+}\right)^{-1}$ is an isometric operator, and, moreover,

$$
\left\|\left(T_{+}\right)^{-1} f^{\prime}\right\|_{1}^{2}=\|f\|_{1}^{2} \leq \delta^{-1}[f, f]_{1} \leq \delta^{-1}\|f\|_{1}^{2} .
$$

Therefore, the closed isometric operator $\left(T_{+}\right)^{-1}$ is also bounded. Consequently, $\operatorname{ran} T_{+}=\operatorname{dom}\left(T_{+}\right)^{-1}$ is a closed subspace in $\mathfrak{H}_{2}$. On the other hand, since dom $T$ admits the decomposition (2.11), where $\mathfrak{T}_{1}$ is finite dimensional and mul $T=\operatorname{mul} T_{+}$ (also finite dimensional), one concludes that

$$
\operatorname{ran} T=T\left(\mathfrak{T}_{1}\right)+\operatorname{ran} T_{+}
$$

as a finite dimensional extension of the closed subspace ran $T_{+}$is a closed subspace of $\mathfrak{H}_{2}$. This completes the proof.

Lemma 2.4 can be seen as an extension of [11, Theorem IX.3.1]. It is known e.g. from [11, Theorem IX.3.2] and [31, Theorems 6.2, 6.3]) that if $T$ is an isometric operator in a Pontryagin space such that $\overline{\operatorname{ran}} T$ (resp. $\overline{\operatorname{dom}} T$ ) is a nondegenerate subspace, then $T$ (resp. $T^{-1}$ ) is continuous. The next lemma contains main properties of isometric relations acting between two Pontryagin spaces.

Lemma 2.5 For an isometric relation $T$ from the Pontryagin space $\left(\mathfrak{H}_{1}, J_{\mathfrak{H}_{1}}\right)$ to the Pontryagin space $\left(\mathfrak{H}_{2}, J_{\mathfrak{H}_{2}}\right)$ the following statements hold:

(i) If $\overline{\operatorname{ran}} T$ (resp. $\overline{\mathrm{dom}} T$ ) is a nondegenerate subspace of $\left(\mathfrak{H}_{2}, J_{\mathfrak{H}_{2}}\right.$ ), then $T$ (resp. $T^{-1}$ ) is a continuous operator.

(ii) If $T$ is densely defined then $\kappa_{-}\left(\mathfrak{H}_{1}\right) \leq \kappa_{-}\left(\mathfrak{H}_{2}\right)$ and if, in addition, $\kappa_{-}\left(\mathfrak{H}_{1}\right)=$ $\kappa_{-}\left(\mathfrak{H}_{2}\right)$, then $\operatorname{ran} \bar{T}$ is a closed nondegenerate subspace of $\mathfrak{H}_{2}$ and, moreover, $T$ and $T^{-1}$ are continuous operators.

(iii) If $\operatorname{ran} T$ is dense in $\mathfrak{H}_{2}$ then $\kappa_{-}\left(\mathfrak{H}_{1}\right) \geq \kappa_{-}\left(\mathfrak{H}_{2}\right)$ and if, in addition, $\kappa_{-}\left(\mathfrak{H}_{1}\right)=$ $\kappa_{-}\left(\mathfrak{H}_{2}\right)$, then $\operatorname{dom} \bar{T}$ is a closed nondegenerate subspace of $\mathfrak{H}_{1}$ and, moreover, $T$ and $T^{-1}$ are continuous operators.

(iv) If the relation $T$ is unitary and $\operatorname{ker} T=\operatorname{mul} T=\{0\}$, then $\kappa_{-}\left(\mathfrak{H}_{1}\right)=\kappa_{-}\left(\mathfrak{H}_{2}\right)$ and $T$ is a standard unitary operator.

(v) If the relation $T$ is unitary and $\kappa_{-}\left(\mathfrak{H}_{1}\right)=\kappa_{-}\left(\mathfrak{H}_{2}\right)$, then

$$
\operatorname{mul} T=\{0\} \quad \Longleftrightarrow \quad \operatorname{ker} T=\{0\} .
$$

In particular, if $\kappa_{-}\left(\mathfrak{H}_{1}\right)=\kappa_{-}\left(\mathfrak{H}_{2}\right)$ then a unitary relation $T$ is an operator if and only if it is a standard unitary operator.

Proof (i) By assumption $T \subseteq T^{-[*]}$ and $\bar{T} \subseteq T^{-[*]}$, i.e., $\bar{T}$ is also isometric. Hence, if $\overline{\operatorname{ran}} T$ is nondegenerate, (2.8) implies

$$
\operatorname{mul} \bar{T} \subset \overline{\operatorname{ran}} T \cap(\overline{\operatorname{ran}} T)^{[\perp]}=\{0\} .
$$

Thus $T$ is a closable operator, which by Lemma 2.4 and the closed graph theorem implies that $\bar{T}$ and, therefore, also $T$ is continuous. Similarly it is seen that

$$
\text { ker } \bar{T} \subseteq \overline{\operatorname{dom}} T \cap(\overline{\operatorname{dom}} T)^{[\perp]}=\{0\},
$$


if $\overline{\operatorname{dom}} T$ is nondegenerate, and then $\bar{T}^{-1}$ and $T^{-1}$ are continuous.

(ii) Since $\overline{\operatorname{dom}} T=\mathfrak{H}_{1}$ is nondegenerate, $T^{-1}$ is a continuous operator by item (i). On the other hand dom $T$, as a dense subspace of $\mathfrak{H}_{1}$, contains a negative subspace $\mathcal{D}_{-} \subset \operatorname{dom} T$ of dimension $\kappa_{-}\left(\mathfrak{H}_{1}\right)$; see [11, Theorem IX.1.4]. Then also $\operatorname{ran} T$ contains a negative subspace of the same dimension and hence $\kappa_{-}\left(\mathfrak{H}_{2}\right) \geq \kappa_{-}\left(\mathfrak{H}_{1}\right)$. Now assume that $\kappa_{-}\left(\mathfrak{H}_{1}\right)=\kappa_{-}\left(\mathfrak{H}_{2}\right)$. Then $\overline{\operatorname{ran}} T=\overline{\operatorname{ran}} \bar{T}$ is necessarily a nondegenerate subspace of the Pontryagin space $\left(\mathfrak{H}_{2}, J_{\mathfrak{H}_{2}}\right)$, see e.g. [11, Lemma II.10.5], and (i) shows that $T$ is a continuous operator.

(iii) This follows by applying (ii) to $T^{-1}$, which is also an isometric relation.

(iv) If $T$ is unitary then the conditions $\operatorname{ker} T=\operatorname{mul} T=\{0\}$ are equivalent to $\overline{\operatorname{dom}} T=\mathfrak{H}_{1}$ and $\overline{\operatorname{ran}} T=\mathfrak{H}_{2}$; see (2.9) in Proposition 2.2. Now the assertions follow from (ii) and (iii).

(v) Let $\kappa_{-}\left(\mathfrak{H}_{1}\right)=\kappa_{-}\left(\mathfrak{H}_{2}\right)$ and let $T$ be unitary. By symmetry it suffices to prove one implication in (2.12), say, “ $\Rightarrow$ ". The condition mul $T=\{0\}$ is equivalent to $\overline{\operatorname{ran}} T=\mathfrak{H}_{2}$; see (2.9). Now item (iii) shows that $T$ and $T^{-1}$ are continuous operators. Thus, in particular, ker $T=\{0\}$ and (2.9) together with Lemma 2.4 shows that $\operatorname{dom} T=\mathfrak{H}_{1}$ and $\operatorname{ran} T=\mathfrak{H}_{2}$.

\subsection{Operator Colligations}

Let $\mathfrak{H}$ be a Pontryagin space, $\mathfrak{L}_{1}$ and $\mathfrak{L}_{2}$ be Hilbert spaces. The set of bounded everywhere defined operators from $\mathfrak{L}_{1}$ to $\mathfrak{L}_{2}$ is denoted by $\mathbf{B}\left(\mathfrak{L}_{1}, \mathfrak{L}_{2}\right), \mathbf{B}\left(\mathfrak{L}_{1}\right):=\mathbf{B}\left(\mathfrak{L}_{1}, \mathfrak{L}_{1}\right)$. Let $U$ be a bounded operator from $\mathfrak{H} \oplus \mathfrak{L}_{1}$ to $\mathfrak{H} \oplus \mathfrak{L}_{2}$ represented in the block form

$$
U=\left(\begin{array}{cc}
T & F \\
G & H
\end{array}\right):\left(\begin{array}{c}
\mathfrak{H} \\
\mathfrak{L}_{1}
\end{array}\right) \rightarrow\left(\begin{array}{c}
\mathfrak{H} \\
\mathfrak{L}_{2}
\end{array}\right)
$$

The quadruple $\left(\mathfrak{H}, \mathfrak{L}_{1}, \mathfrak{L}_{2}, U\right)$ is called a colligation, $\mathfrak{H}$ is the state space, $\mathfrak{L}_{1}$ and $\mathfrak{L}_{2}$ are the incoming and the outgoing spaces, $T$ is the main operator and $U$ is called the connecting operator of the colligation. The colligation $\left(\mathfrak{H}, \mathfrak{L}_{1}, \mathfrak{L}_{2}, U\right)$ is called unitary, if $U$ is a unitary operator from $\mathfrak{H} \oplus \mathfrak{L}_{1}$ to $\mathfrak{H} \oplus \mathfrak{L}_{2}$. The colligation $\left(\mathfrak{H}, \mathfrak{L}_{2}, \mathfrak{L}_{1}, U^{[*]}\right)$ is called adjoint to the colligation $\left(\mathfrak{H}, \mathfrak{L}_{1}, \mathfrak{L}_{2}, U\right)$; cf. [1,12].

Components of a unitary colligation satisfy the following identities

$$
\begin{aligned}
& T^{[*]} T+G^{[*]} G=I_{\mathfrak{H}}, F^{[*]} F+H^{*} H=I_{\mathfrak{L}_{1}}, T^{[*]} F+G^{[*]} H=0, \\
& T T^{[*]}+F F^{[*]}=I_{\mathfrak{H}}, \quad G G^{[*]}+H H^{*}=I_{\mathfrak{L}_{2}}, T G^{[*]}+F H^{*}=0,
\end{aligned}
$$

which are equivalent to the identities

$$
U^{[*]} U=I_{\mathfrak{H} \oplus \mathfrak{L}_{1}}, \quad U U^{[*]}=I_{\mathfrak{H} \oplus \mathfrak{L}_{2}} .
$$

A colligation $\left(\mathfrak{H}, \mathfrak{L}_{1}, \mathfrak{L}_{2}, U\right)$ is said to be closely connected, if

$$
\mathfrak{H}=\overline{\operatorname{span}}\left\{\operatorname{ran}\left(T^{m} F\right), \operatorname{ran}\left(\left(T^{[*]}\right)^{n} G^{[*]}\right): m, n \geq 0\right\} .
$$


A unitary colligation is closely connected if and only if the operator $U$ has no nontrivial reducing subspaces. The operator valued function

$$
\Theta_{\Delta}(\lambda):=H+\lambda G(I-\lambda T)^{-1} F, \quad 1 / \lambda \in \rho(T),
$$

is called the characteristic function of the unitary colligation $\left(\mathfrak{H}, \mathfrak{L}_{1}, \mathfrak{L}_{2}, U\right)$.

Recall, see e.g. [1], that a $\mathbf{B}\left(\mathfrak{L}_{1}, \mathfrak{L}_{2}\right)$-valued function $\Theta(\lambda)$ is said to belong to the generalized Schur class $\mathcal{S}_{\kappa}^{0}\left(\mathfrak{L}_{1}, \mathfrak{L}_{2}\right)$ if it is holomorphic in a neighborhood $\Omega$ of 0 and the kernel

$$
\mathrm{K}_{\omega}^{\Theta}(\lambda)=\frac{I-\Theta(\lambda) \Theta(\omega)^{*}}{1-\lambda \bar{\omega}}
$$

has $\kappa$ negative squares in $\Omega \times \Omega$, i.e. for any finite set of points $\omega_{1}, \ldots, \omega_{n}$ in $\Omega$ and vectors $f_{1}, \ldots, f_{n}$ in $\mathfrak{L}_{2}$, the Hermitian matrix

$$
\left(\left(\mathrm{K}_{\omega_{i}}^{\Theta}\left(\omega_{j}\right) f_{j}, f_{i}\right)_{\mathfrak{L}_{2}}\right)_{i, j=1}^{n}
$$

has at most $\kappa$ negative eigenvalues, and for some choice of $\omega_{1}, \ldots, \omega_{n}$ in $\Omega$ and $f_{1}, \ldots, f_{n}$ in $\mathfrak{L}_{2}$ the matrix (2.16) has exactly $\kappa$ negative eigenvalues.

As is known, see [1], the characteristic function of a closely connected unitary colligation belongs to the generalized Schur class $\mathcal{S}_{\kappa}^{0}\left(\mathfrak{L}_{1}, \mathfrak{L}_{2}\right)$, where $\kappa=\kappa_{-}(\mathfrak{H})$. Moreover, the converse is also true; see e.g. [1, Theorem 2.3.1].

Theorem 2.6 Let $\mathfrak{L}_{1}$ and $\mathfrak{L}_{2}$ be Hilbert spaces and let $S(\lambda)$ belong to the generalized Schur class $\mathcal{S}_{\kappa}^{0}\left(\mathfrak{L}_{1}, \mathfrak{L}_{2}\right)$. Then there exists a closely connected unitary colligation $\left(\mathfrak{H}, \mathfrak{L}_{1}, \mathfrak{L}_{2}, U\right)$, such that the corresponding characteristic function $\Theta_{\Delta}(\lambda)$ coincides with $S(\lambda)$ in a neighborhood $\Omega$ of 0 .

In what follows a $\mathbf{B}\left(\mathfrak{L}_{1}, \mathfrak{L}_{2}\right)$-valued function $\Theta(\cdot)$ holomorphic in some open subset $\Omega \subset \mathbb{D}$ is said to belong to the generalized Schur class $\mathcal{S}_{\kappa}\left(\mathfrak{L}_{1}, \mathfrak{L}_{2}\right)$, if the kernel (2.15) has $\kappa$ negative squares in $\Omega \times \Omega$. In particular, we do not require that $0 \in \Omega$, which implies that characteristic functions of unitary colligations used in Theorem 2.6 are not sufficient to give a realization for all functions $\Theta(\cdot)$ from the class $\mathcal{S}_{K}\left(\mathfrak{L}_{1}, \mathfrak{L}_{2}\right)$. In the next section we introduce the notions of a unitary boundary pair for an isometric operator and an associated Weyl function as a replacement for unitary colligations and their characteristic functions. These new notions allow to realize an arbitrary operator function from the class $\mathcal{S}_{K}\left(\mathfrak{L}_{1}, \mathfrak{L}_{2}\right)$ as the Weyl function of a Pontryagin space isometric operator, corresponding to some unitary boundary pair.

\section{Unitary Boundary Pairs for Isometric Operators}

\subsection{Unitary Boundary Pairs and the Main Transform}

Let $\mathfrak{H}$ be a Pontryagin space with the negative index $\kappa$ and the fundamental symmetry $J_{\mathfrak{H}}$ and let $\mathfrak{L}_{1}$ and $\mathfrak{L}_{2}$ be Hilbert spaces. In this section we introduce the notion of $a$ 
unitary boundary pair for an isometric operator $V$ : here, and in what follows, $V$ is assumed to be closed.

For this purpose equip the Hilbert spaces $\mathfrak{H}^{2}$ and $\mathfrak{L}=\mathfrak{L}_{1} \times \mathfrak{L}_{2}$ with the indefinite inner products by the formulas

$$
[\widehat{f}, \widehat{g}]_{\mathfrak{H}^{2}}=\left(J_{\mathfrak{H}^{2}} \widehat{f}, \widehat{g}\right)_{\mathfrak{H}^{2}}, \quad[\widehat{u}, \widehat{v}]_{\mathfrak{L}}=\left(J_{\mathfrak{L}} \widehat{u}, \widehat{v}\right)_{\mathfrak{L}}
$$

where

$$
J_{\mathfrak{H}^{2}}=\left(\begin{array}{cc}
J_{\mathfrak{H}} & 0 \\
0 & -J_{\mathfrak{H}}
\end{array}\right), \quad J_{\mathfrak{L}}=\left(\begin{array}{cc}
I_{\mathfrak{L}_{1}} & 0 \\
0 & -I_{\mathfrak{L}_{2}}
\end{array}\right)
$$

and $\widehat{f}=\left\{f, f^{\prime}\right\}, \widehat{g}=\left\{g, g^{\prime}\right\} \in \mathfrak{H}^{2}, \widehat{u}=\left\{u_{1}, u_{2}\right\}, \widehat{v}=\left\{v_{1}, v_{2}\right\} \in \mathfrak{L}$. Then $\left(\mathfrak{H}^{2}, J_{\mathfrak{H}^{2}}\right)$ and $\left(\mathfrak{L}, J_{\mathfrak{L}}\right)$ are Kre rn spaces. In particular, for $\mathfrak{V} \subset \mathfrak{H}^{2}$ the linear set $\mathfrak{V}^{[\perp]}$ in the Kreı̆n space $\left(\mathfrak{H}^{2}, J_{\mathfrak{H}^{2}}\right)$ can be characterized as follows

$$
\widehat{g} \in \mathfrak{V}^{[\perp]} \Longleftrightarrow[\widehat{f}, \widehat{g}]_{\mathfrak{H}^{2}}=0 \text { for all } \widehat{f} \in \mathfrak{V} \Longleftrightarrow \widehat{g} \in \mathfrak{V}^{-[*]}
$$

Definition 3.1 Let $\mathfrak{L}_{1}$ and $\mathfrak{L}_{2}$ be Hilbert spaces, let $V$ be a closed isometric operator (or isometric relation) in $\mathfrak{H}$ and let $\Gamma$ be a linear relation $\mathfrak{H}^{2} \rightarrow \mathfrak{L}$, where $\mathfrak{L}:=\mathfrak{L}_{1} \times \mathfrak{L}_{2}$.

The pair $(\mathfrak{L}, \Gamma)$ will be called a unitary boundary pair for $V$ if:

(1) $V=\operatorname{ker} \Gamma$ and for all $\{\widehat{f}, \widehat{u}\},\{\widehat{g}, \widehat{v}\} \in \Gamma$ the following identity holds

$$
[f, g]_{\mathfrak{H}}-\left[f^{\prime}, g^{\prime}\right]_{\mathfrak{H}}=\left(u_{1}, v_{1}\right) \mathfrak{L}_{1}-\left(u_{2}, v_{2}\right) \mathfrak{L}_{2}
$$

(2) $\Gamma$ is maximal in the sense that if $\{\widehat{g}, \widehat{v}\} \in \mathfrak{H}^{2} \times \mathfrak{L}$ satisfies (3.2) for all $\{\widehat{f}, \widehat{u}\} \in \Gamma$, then $\{\widehat{g}, \widehat{v}\} \in \Gamma$. Here

$$
\{\widehat{f}, \widehat{u}\}=\left\{\left(\begin{array}{c}
f \\
f^{\prime}
\end{array}\right),\left(\begin{array}{c}
u_{1} \\
u_{2}
\end{array}\right)\right\}, \quad\{\widehat{g}, \widehat{v}\}=\left\{\left(\begin{array}{c}
g \\
g^{\prime}
\end{array}\right),\left(\begin{array}{c}
v_{1} \\
v_{2}
\end{array}\right)\right\} \in \mathfrak{H}^{2} \times \mathfrak{L}
$$

Item (1) of Definition 3.1 means that $\Gamma$ is an isometric linear relation from the Kreın space $\left(\mathfrak{H}^{2}, J_{\mathfrak{H}^{2}}\right)$ to the Kreı̆n space $\left(\mathfrak{L}, J_{\mathfrak{L}}\right)$, while items (1) and (2) together mean that $\Gamma$ is unitary.

Application of Proposition 2.2 to a unitary boundary pair leads to the following statement.

Proposition 3.2 Let $(\mathfrak{L}, \Gamma)$ be a unitary boundary pair for $V$. Then:

(i) $V_{*}:=\operatorname{dom} \Gamma$ is dense in $V^{-[*]}$;

(ii) ran $\Gamma$ is dense in $\mathfrak{L}$ if and only if mul $\Gamma=\{0\}$;

(iii) $\operatorname{ran} \Gamma=\mathfrak{L}$ if and only if $\operatorname{dom} \Gamma=V^{-[*]}$ and mul $\Gamma=\{0\}$. 
Define the components $\Gamma_{1}$ and $\Gamma_{2}$ of $\Gamma$ by

$$
\begin{aligned}
& \Gamma_{1}:=\left\{\left\{\widehat{f}, u_{1}\right\}:\left\{\widehat{f},\left(\begin{array}{l}
u_{1} \\
u_{2}
\end{array}\right)\right\} \in \Gamma \quad \text { for some } u_{2} \in \mathfrak{L}_{2}\right\} \\
& \Gamma_{2}:=\left\{\left\{\widehat{f}, u_{2}\right\}:\left\{\widehat{f},\left(\begin{array}{l}
u_{1} \\
u_{2}
\end{array}\right)\right\} \in \Gamma \text { for some } u_{1} \in \mathfrak{L}_{1}\right\} .
\end{aligned}
$$

In the case that $\Gamma_{1}$ and $\Gamma_{2}$ are single-valued, i.e. mul $\Gamma_{1}=\operatorname{mul} \Gamma_{2}=0$ and $\operatorname{dom} \Gamma=$ $V^{-[*]}$ and $\operatorname{ran} \Gamma=\mathfrak{L}_{1} \times \mathfrak{L}_{2}$ the collection $\left\{\mathfrak{L}_{1} \times \mathfrak{L}_{2}, \Gamma_{1}, \Gamma_{2}\right\}$ is called an ordinary boundary triple for the isometric operator $V$. For a Hilbert space isometric operator the corresponding notion was introduced and studied in [41,42] as a boundary triple for the dual pair $\left(V, V^{-1}\right)$.

For ordinary boundary triples an application of the closed graph theorem shows that the component mappings $\Gamma_{1}$ and $\Gamma_{2}$ are bounded. However, for a general unitary boundary pair $(\mathfrak{L}, \Gamma)$ the mappings $\Gamma_{1}$ and $\Gamma_{2}$ need not be bounded or single-valued. With $\Gamma_{1}$ and $\Gamma_{2}$ one associates the extensions $V_{1}$ and $V_{2}$ of $V$ by the equalities

$$
V_{1}:=\operatorname{ker} \Gamma_{1}, \quad V_{2}:=\operatorname{ker} \Gamma_{2}
$$

It follows from the identity (3.2) that $V_{1}$ is an expanding linear relation and $V_{2}$ is a contractive linear relation in the Pontryagin space $\mathfrak{H}$. Moreover, it is clear from (3.2) that the sets ker $V_{1} \backslash \operatorname{ker} V$ and mul $V_{2}\left(=\right.$ mul $V_{2} \backslash$ mul $\left.V\right)$ consist of negative vectors in $\mathfrak{H}$ while the sets ker $V_{2} \backslash \operatorname{ker} V$ and mul $V_{1}\left(=\operatorname{mul} V_{1} \backslash\right.$ mul $\left.V\right)$ consist of positive vectors of $\mathfrak{H}$.

Let $\mathfrak{H}_{j}:=\mathfrak{H} \times \mathfrak{L}_{j}(j=1,2)$ be a Pontryagin space with the inner product

$$
\left[\left(\begin{array}{l}
f \\
u
\end{array}\right),\left(\begin{array}{l}
f \\
u
\end{array}\right)\right]_{\mathfrak{H}_{j}}=[f, f]_{\mathfrak{H}}+\|u\|_{\mathfrak{L}_{j}}^{2}, \quad f \in \mathfrak{H}, u \in \mathfrak{L}_{j}, j=1,2
$$

In establishing some properties of unitary boundary pairs it is useful to connect the unitary relation $\Gamma$ which acts between two Kreı̆n spaces to another unitary relation that acts between two Pontryagin spaces, since unitary relations between Pontryagin spaces have simpler structure. For this purpose we introduce the following transform from the Kreı̆n space $\left(\mathfrak{H}^{2} \oplus \mathfrak{L}, J_{\mathfrak{H}^{2}} \oplus\left(-J_{\mathfrak{L}}\right)\right)$ to the Krĕ̌n space $\left(\mathfrak{H}_{2} \oplus \mathfrak{H}_{1},\left(J_{\mathfrak{H}_{2}}\right) \oplus\left(-J_{\mathfrak{H}_{1}}\right)\right)$ by

$$
\mathcal{J}:\left\{\left(\begin{array}{c}
f \\
f^{\prime}
\end{array}\right),\left(\begin{array}{c}
u_{1} \\
u_{2}
\end{array}\right)\right\} \mapsto\left\{\left(\begin{array}{c}
f \\
u_{2}
\end{array}\right),\left(\begin{array}{c}
f^{\prime} \\
u_{1}
\end{array}\right)\right\}, \quad f, f^{\prime} \in \mathfrak{H} \text { and } u_{1} \in \mathfrak{L}_{1}, u_{2} \in \mathfrak{L}_{2}
$$

It establishes a one-to-one correspondence between (closed) linear relations $\Gamma$ from $\left(\mathfrak{H}^{2}, J_{\mathfrak{H}_{2}}\right)$ to $\left(\mathfrak{L}, J_{\mathfrak{L}}\right)$ and (closed) linear relations $\mathcal{U}$ from $\mathfrak{H}_{2}$ to $\mathfrak{H}_{1}$ via

$$
\Gamma \mapsto \mathcal{U}:=\mathcal{J}(\Gamma)=\left\{\left\{\left(\begin{array}{c}
f \\
u_{2}
\end{array}\right),\left(\begin{array}{c}
f^{\prime} \\
u_{1}
\end{array}\right)\right\}:\left\{\left(\begin{array}{c}
f \\
f^{\prime}
\end{array}\right),\left(\begin{array}{l}
u_{1} \\
u_{2}
\end{array}\right)\right\} \in \Gamma\right\}
$$


The linear relation $\mathcal{U}$ will be called the main transform of $\Gamma$; $\mathrm{cf}$. [19] for the case of symmetric operators. In the following lemma, which is an analog of [19, Proposition 2.10], some basic properties of the transform $\mathcal{J}$ are given.

Lemma 3.3 Let the linear relation $\Gamma$ from $\left(\mathfrak{H}^{2}, J_{\mathfrak{H}^{2}}\right)$ to $\left(\mathfrak{L}, J_{\mathfrak{L}}\right)$ and the linear relation $\mathcal{U}$ from $\mathfrak{H} \times \mathfrak{L}_{2}$ to $\mathfrak{H} \times \mathfrak{L}_{1}$ be connected by $\mathcal{U}=\mathcal{J}(\Gamma)$. Then

$$
\mathcal{U}^{-[*]}=\mathcal{J}\left(\Gamma^{-[*]}\right)
$$

Moreover, the transform $\mathcal{J}$ establishes a one-to-one correspondence between isometric (unitary, contractive, expanding) relations $\Gamma$ from $\left(\mathfrak{H}^{2}, J_{\mathfrak{H}^{2}}\right)$ to $\left(\mathfrak{L}, J_{\mathfrak{L}}\right)$ and isometric (unitary, contractive, expanding) relations $\mathcal{U}$ from $\mathfrak{H} \times \mathfrak{L}_{2}$ to $\mathfrak{H} \times \mathfrak{L}_{1}$.

Proof It is straightforward to check that for all elements of the form

$$
\left\{\left(\begin{array}{c}
f \\
f^{\prime}
\end{array}\right),\left(\begin{array}{c}
u_{1} \\
u_{2}
\end{array}\right)\right\},\left\{\left(\begin{array}{c}
g \\
g^{\prime}
\end{array}\right),\left(\begin{array}{c}
v_{1} \\
v_{2}
\end{array}\right)\right\} \in\left(\begin{array}{c}
\mathfrak{H} \\
\mathfrak{H}
\end{array}\right) \times\left(\begin{array}{c}
\mathfrak{L}_{1} \\
\mathfrak{L}_{2}
\end{array}\right)
$$

the following identity is satisfied:

$$
\left[\left(\begin{array}{c}
f \\
u_{2}
\end{array}\right),\left(\begin{array}{c}
g \\
v_{2}
\end{array}\right)\right]_{\mathfrak{H}_{2}}-\left[\left(\begin{array}{c}
f^{\prime} \\
u_{1}
\end{array}\right),\left(\begin{array}{c}
g^{\prime} \\
v_{1}
\end{array}\right)\right]_{\mathfrak{H}_{1}}=\left[\left(\begin{array}{c}
f \\
f^{\prime}
\end{array}\right),\left(\begin{array}{c}
g \\
g^{\prime}
\end{array}\right)\right]_{\mathfrak{H}^{2}}-\left[\left(\begin{array}{l}
u_{1} \\
u_{2}
\end{array}\right),\left(\begin{array}{l}
v_{1} \\
v_{2}
\end{array}\right)\right]_{\mathfrak{L}} .
$$

In view of (3.1) this identity implies the equivalence

$$
\left\{\left(\begin{array}{c}
f \\
u_{2}
\end{array}\right),\left(\begin{array}{c}
f^{\prime} \\
u_{1}
\end{array}\right)\right\} \in \mathcal{U}^{-[*]} \Longleftrightarrow\left\{\left(\begin{array}{c}
f \\
f^{\prime}
\end{array}\right),\left(\begin{array}{l}
u_{1} \\
u_{2}
\end{array}\right)\right\} \in \Gamma^{-[*]}
$$

which leads to identity (3.7). It follows from (3.7) that

$$
\mathcal{U}^{-1} \subseteq \mathcal{U}^{[*]} \Longleftrightarrow \Gamma^{-1} \subseteq \Gamma^{[*]}, \quad \mathcal{U}^{-1}=\mathcal{U}^{[*]} \Longleftrightarrow \Gamma^{-1}=\Gamma^{[*]},
$$

i.e., $\mathcal{U}$ is isometric (unitary) precisely when $\Gamma$ is isometric (resp. unitary). The connection between contractive (expanding) relations $\Gamma$ and $\mathcal{U}$ is clear from (3.8).

The next proposition contains the basic properties of $\Gamma_{1}, \Gamma_{2}$ and $V_{1}, V_{2}$ for a unitary boundary pair $(\mathfrak{L}, \Gamma)$.

Proposition 3.4 Let $(\mathfrak{L}, \Gamma)$ be a unitary boundary pair for $V$, let $\Gamma_{1}, \Gamma_{2}$ and $V_{1}, V_{2}$ be defined by (3.3), (3.4) and (3.5), and let $\mathcal{U}$ be the main transform of $\Gamma$. Then:

(i) $\Gamma_{1}$ and $\Gamma_{2}$ are closed;

(ii) $\operatorname{ran} \Gamma_{1}=\mathfrak{L}_{1}$ and $\operatorname{ran} \Gamma_{2}=\mathfrak{L}_{2}$;

(iii) $\operatorname{mul} \Gamma_{j}=P_{\mathfrak{L}_{j}}(\operatorname{mul} \Gamma), j=1,2$, and the following equivalences hold:

$$
\operatorname{mul} \Gamma_{1}=\{0\} \quad \Longleftrightarrow \operatorname{mul} \Gamma_{2}=\{0\} \quad \Longleftrightarrow \operatorname{mul} \Gamma=\{0\} ;
$$


(iv) the extensions $V_{1}$ and $V_{2}$ of $V$ are closed, $V_{1} \subseteq V_{2}^{-[*]}$ and $V_{2} \subseteq V_{1}^{-[*]}$;

(v) the following equivalences hold:

$$
\begin{aligned}
& \operatorname{mul} V_{2}=\{0\} \Longleftrightarrow \operatorname{mul} \mathcal{U}=\{0\} ; \\
& \text { ker } V_{1}=\{0\} \Longleftrightarrow \operatorname{ker} \mathcal{U}=\{0\} ; \\
& \operatorname{mul} V_{2}=\{0\} \Longleftrightarrow \operatorname{ker} V_{1}=\{0\}
\end{aligned}
$$

If one of the sets appearing in the above equivalences is trivial, then the main transform $\mathcal{U}$ from $\mathfrak{H} \times \mathfrak{L}_{2}$ to $\mathfrak{H} \times \mathfrak{L}_{1}$ of $\Gamma$ is a standard unitary operator.

Proof (i) To prove that $\Gamma_{1}$ is closed assume that $\left\{\widehat{f}_{n}, \widehat{u}_{n}\right\} \in \Gamma$ such that $\widehat{f}_{n} \rightarrow \widehat{f} \in \mathfrak{H}^{2}$ and $u_{1, n} \rightarrow u_{1} \in \mathfrak{L}_{1}$. Then (3.2) gives

$$
\begin{aligned}
& {\left[f_{n}-f_{m}, f_{n}-f_{m}\right]_{\mathfrak{H}}-\left[f_{n}^{\prime}-f_{m}^{\prime}, f_{n}^{\prime}-f_{m}^{\prime}\right]_{\mathfrak{H}}} \\
& \quad=\left\|u_{1, n}-u_{1, m}\right\|_{\mathfrak{L}_{1}}^{2}-\left\|u_{2, n}-u_{2, m}\right\|_{\mathfrak{L}_{2}}^{2}
\end{aligned}
$$

and letting $n, m \rightarrow \infty$ one concludes that $\left\|u_{2, n}-u_{2, m}\right\|_{\mathfrak{L}_{2}} \rightarrow 0$. As a Cauchy sequence $\left(u_{2, n}\right)$ converges to some element $u_{2}$ in $\mathfrak{L}_{2}$. This means that $\left\{\widehat{f}_{n}, \widehat{u}_{n}\right\} \rightarrow$ $\{\widehat{f}, \widehat{u}\}$ and, since $\Gamma$ is closed as a unitary relation, $\{\widehat{f}, \widehat{u}\} \in \Gamma$ and thus $\left\{\widehat{f}, u_{1}\right\} \in$ $\Gamma_{1}$. This proves that $\Gamma_{1}$ is closed. Similarly one proves that $\Gamma_{2}$ is closed.

(ii) First it is shown that $\operatorname{ran} \Gamma_{1}$ is a closed subspace of $\mathfrak{L}_{1}$. For this consider the main transform $\mathcal{U}=\mathcal{J}(\Gamma)$. By Lemma $3.3 \mathcal{U}$ is a unitary relation between the Pontryagin spaces $\mathfrak{H} \times \mathfrak{L}_{2}$ and $\mathfrak{H} \times \mathfrak{L}_{1}$. Moreover, by Lemma 2.4 ran $\mathcal{U}$ is closed and Proposition 2.2 shows that mul $\mathcal{U}$ is the isotropic part of $\operatorname{ran} \mathcal{U}$. Therefore mulU $\mathcal{U}$ is a closed finite dimensional subspace of $\mathfrak{H} \times \mathfrak{L}_{1}$ and thus also the codimension $k$ of $\operatorname{ran} \mathcal{U}$ is finite $\left(k \leq \kappa_{-}(\mathfrak{H})\right)$. Let $\mathfrak{M}$ be any $k$-dimensional subspace such that $\operatorname{ran} \mathcal{U}+\mathfrak{M}=\mathfrak{H} \times \mathfrak{L}_{1}$ and let $P_{1}$ be the orthogonal projection from $\mathfrak{H} \times \mathfrak{L}_{1}$ onto $\mathfrak{L}_{1}$. Then

$$
\mathfrak{L}_{1}=P_{1}(\operatorname{ran} \mathcal{U} \dot{+} \mathfrak{M})=P_{1} \operatorname{ran} \mathcal{U}+P_{1} \mathfrak{M}
$$

and here $\operatorname{dim} P_{1} \mathfrak{M} \leq k$, which implies that $P_{1} \operatorname{ran} \mathcal{U}=\operatorname{ran} \Gamma_{1}$ is closed.

To see that $\operatorname{ran} \Gamma_{1}=\mathfrak{L}_{1}$ it suffices to prove that ran $\Gamma_{1}$ is dense in $\mathfrak{L}_{1}$. For this assume that $v_{1} \perp \operatorname{ran} \Gamma_{1}$. Let $\widehat{g}=\{0,0\} \in \mathfrak{H}^{2}$ and $\widehat{v}=\left\{v_{1}, 0\right\} \in \mathfrak{L}$. Then $\{\widehat{g}, \widehat{v}\}$ satisfies the identity (3.2) for all $\{\widehat{f}, \widehat{u}\} \in \Gamma$, and hence assumption (2) in Definition 3.1 implies that $\{\widehat{g}, \widehat{v}\} \in \Gamma$. This means that $\widehat{v} \in \operatorname{mul} \Gamma$ and then, in particular, $v_{1} \in \operatorname{mul} \Gamma_{1} \subseteq \operatorname{ran} \Gamma_{1}$. Thus, $v_{1}=0$ and this proves that $\operatorname{ran} \Gamma_{1}=\mathfrak{L}_{1}$. The equality $\operatorname{ran} \Gamma_{2}=\mathfrak{L}_{2}$ is then clear by symmetry.

(iii) The identities mul $\Gamma_{j}=P_{\mathfrak{L}_{j}}(\operatorname{mul} \Gamma), j=1,2$, are clear from the definition of $\Gamma$. Hence, mul $\Gamma=\{0\}$ implies that mul $\Gamma_{1}=$ mul $\Gamma_{2}=\{0\}$. Conversely, assume that e.g. mul $\Gamma_{1}=\{0\}$ and that $\widehat{v} \in \operatorname{mul} \Gamma$. Then $\widehat{v}=\left\{0, v_{2}\right\}$ and hence for all $\{\widehat{f}, \widehat{u}\} \in \Gamma$ the identity (3.2) implies that

$$
0=\left(u_{1}, 0\right) \mathfrak{L}_{1}-\left(u_{2}, v_{2}\right)_{\mathfrak{L}_{2}}=-\left(u_{2}, v_{2}\right) \mathfrak{L}_{2} .
$$


In item (ii) it was shows that $\operatorname{ran} \Gamma_{2}=\mathfrak{L}_{2}$ and, thus, one concludes that $v_{2}=0$. Hence, mul $\Gamma=\{0\}$. Similarly, mul $\Gamma_{2}=\{0\}$ implies mul $\Gamma=\{0\}$.

(iv) Since $V_{j}=\operatorname{ker} \Gamma_{j}$ and $\Gamma_{j}$ is closed by item (i) also $V_{j}$ is closed $j=1,2$. If $\widehat{f} \in V_{1}$ and $\widehat{g} \in V_{2}$ then it follows from (3.2) that $[\widehat{f}, \widehat{g}]_{\mathfrak{H}^{2}}=0$ and in view of (3.1) this means that the inclusions $V_{1} \subseteq V_{2}^{-[*]}$ and $V_{2} \subseteq V_{1}^{-[*]}$ hold; these inclusions are clearly equivalent to each other.

(v) The definition in (3.6) shows that

$$
\operatorname{mul} V_{2}=P_{\mathfrak{H}}(\operatorname{mul} \mathcal{U})
$$

Hence, mul $\mathcal{U}=\{0\}$ implies mul $V_{2}=\{0\}$. Conversely, if $\left\{f^{\prime}, u_{1}\right\} \in \operatorname{mul} \mathcal{U}$ then $f^{\prime} \in$ mul $V_{2}$ and if mul $V_{2}=\{0\}$ then $f^{\prime}=0$. Now (3.2) implies that $\left(u_{1}, u_{1}\right)_{\mathfrak{L}_{1}}=0$ and thus also $u_{1}=0$, i.e., mulU $\mathcal{U}=\{0\}$. The equivalence of $\operatorname{ker} \mathcal{U}=\{0\}$ and ker $V_{1}=\{0\}$ can be seen in the same way. As to the last equivalence notice that $\kappa_{-}\left(\mathfrak{H} \times \mathfrak{L}_{1}\right)=\kappa_{-}(\mathfrak{H})=\kappa_{-}\left(\mathfrak{H} \times \mathfrak{L}_{2}\right)$. Now, according to item (v) in Lemma $2.5 \operatorname{ker} \mathcal{U}=\{0\}$ is equivalent to mulU $=\{0\}$ and in this case $\mathcal{U}$ is a standard unitary operator.

Later it is shown that the inclusions in (iv) of Proposition 3.4 actually hold as equalities; see Theorem 3.15. In the special case that $\mathfrak{H}$ is a Hilbert space and $V$ is an isometry in $\mathfrak{H}$ Proposition 3.4 can be specialized as follow.

Corollary 3.5 Let $(\mathfrak{L}, \Gamma)$ be a unitary boundary pair for an isometric operator $V$ in the Hilbert space $\mathfrak{H}$. Then the properties (i)-(iv) in Proposition 3.4 hold and, moreover, $V_{2}$ and $V_{1}^{-1}$ are contractive operators. Furthermore, the main transform $\mathcal{U}$ from $\mathfrak{H} \times \mathfrak{L}_{2}$ to $\mathfrak{H} \times \mathfrak{L}_{1}$ of $\Gamma$ is a standard Hilbert space unitary operator.

Proof The fact that in the Hilbert space case $V_{2}$ and $V_{1}^{-1}$ are contractive operators follows from (3.2) which with the choice $\widehat{f}=\widehat{g}$ and $\widehat{u}=\widehat{v}$ can be rewritten as

$$
\|f\|_{\mathfrak{H}}^{2}-\left\|f^{\prime}\right\|_{\mathfrak{H}}^{2}=\left\|u_{1}\right\|_{\mathfrak{L}_{1}}^{2}-\left\|u_{2}\right\|_{\mathfrak{L}_{2}}^{2}
$$

In particular, with $u_{1}=0$ the condition $f^{\prime}=0$ implies $f=0$ and hence ker $V_{1}=\{0\}$. Similarly with $u_{2}=0$ the condition $f=0$ implies $f^{\prime}=0$ and thus mul $V_{2}=\{0\}$. According to item (v) in Proposition 3.4 one has mulU $=\{0\}$ and thus $\mathcal{U} \in \mathbf{B}(\mathfrak{H})$ is a standard unitary operator.

\subsection{The Weyl Function and the $\gamma$-fields of a Unitary Boundary Pair}

Define the set $\mathfrak{N}_{\lambda}\left(V_{*}\right)$ as the intersection of $\mathfrak{N}_{\lambda}$ and $V_{*}$ :

$$
\mathfrak{N}_{\lambda}\left(V_{*}\right):=\left\{f_{\lambda}:\left(\begin{array}{c}
f_{\lambda} \\
\lambda f_{\lambda}
\end{array}\right) \in V_{*}\right\}\left(\subseteq \mathfrak{N}_{\lambda}=\left\{f_{\lambda}:\left(\begin{array}{c}
f_{\lambda} \\
\lambda f_{\lambda}
\end{array}\right) \in V^{-[*]}\right\}\right)
$$

and the corresponding subset of $V_{*}$ :

$$
\widehat{\mathfrak{N}}_{\lambda}\left(V_{*}\right)=\left\{\widehat{f_{\lambda}}=\left(\begin{array}{c}
f_{\lambda} \\
\lambda f_{\lambda}
\end{array}\right): \widehat{f_{\lambda}} \in V_{*}\right\} .
$$


If $\widehat{f}_{\lambda} \in \widehat{\mathfrak{N}}_{\lambda}\left(V_{*}\right)$ and $\widehat{f}_{\mu} \in \widehat{\mathfrak{N}}_{\mu}\left(V_{*}\right)$ then for some $\widehat{u}, \widehat{v} \in \mathfrak{L}$ one has

$$
\left\{\widehat{f_{\lambda}},\left(\begin{array}{l}
u_{1} \\
u_{2}
\end{array}\right)\right\} \in \Gamma, \quad\left\{\widehat{f_{\mu}},\left(\begin{array}{l}
v_{1} \\
v_{2}
\end{array}\right)\right\} \in \Gamma
$$

and an application of (3.2) shows that

$$
(1-\lambda \bar{\mu})\left[f_{\lambda}, f_{\mu}\right]_{\mathfrak{H}}=\left(u_{1}, v_{1}\right) \mathfrak{L}_{1}-\left(u_{2}, v_{2}\right) \mathfrak{L}_{2}
$$

Lemma 3.6 (cf. [16, Lemma 3.2]) Let $u_{j}$ belong to a Hilbert space $\mathcal{H}$, let $\lambda_{j} \in \mathbb{C}$, and define the vector valued function $f_{j}$ by

$$
f_{j}(\lambda):=\frac{u_{j}}{1-\lambda_{j} \lambda}, \quad j=1, \ldots, n
$$

Then the Gram matrix of the functions $f_{j}(\lambda)(j=1, \ldots, n)$ in the space $L_{2}(\mathcal{H})$ over the unit circle

(i) is equal to $G=\left(\frac{\left(u_{j}, u_{k}\right)_{\mathcal{H}}}{1-\lambda_{j} \bar{\lambda}_{k}}\right)_{j, k=1}^{n}$, if $\lambda_{j} \in \mathbb{D}(j, k=1, \ldots, n)$;

(ii) is equal to $-G=\left(-\frac{\left(u_{j}, u_{k}\right) \mathcal{H}}{1-\lambda_{j} \bar{\lambda}_{k}}\right)_{j, k=1}^{n}$, if $\lambda_{j} \in \mathbb{D}_{e}(j, k=1, \ldots, n)$.

Proof To determine the Gram matrix consider the inner product of the $\mathcal{H}$-valued functions $f_{j}(\lambda)$ and $f_{k}(\lambda)$ with $\lambda=e^{i t}, t \in[0,2 \pi]$, for $j, k=1, \ldots, n$.

(i) If $\lambda_{j} \in \mathbb{D}$ then in view of the equalities

$$
\begin{aligned}
\left(f_{j}, f_{k}\right)_{L_{2}(\mathcal{H})} & =\frac{1}{2 \pi} \int_{0}^{2 \pi} \frac{d t}{\left(1-\lambda_{j} e^{i t}\right)\left(1-\bar{\lambda}_{k} e^{-i t}\right)}\left(u_{j}, u_{k}\right)_{\mathcal{H}} \\
& =\frac{1}{2 \pi i} \oint \frac{d \lambda}{\left(1-\lambda_{j} \lambda\right)\left(\lambda-\bar{\lambda}_{k}\right)}\left(u_{j}, u_{k}\right)_{\mathcal{H}}=\frac{\left(u_{j}, u_{k}\right)_{\mathcal{H}}}{1-\lambda_{j} \bar{\lambda}_{k}}
\end{aligned}
$$

the matrix $G$ coincides with the Gram matrix of the functions $f_{j}(\lambda)(j=1, \ldots, n)$ in the space $L_{2}(\mathcal{H})$ on the unit circle.

(ii) Analogously, if $\lambda_{j} \in \mathbb{D}_{e}(j=1, \ldots, n)$ then it follows from

$$
\frac{1}{2 \pi i} \oint \frac{d \lambda}{\left(1-\lambda_{j} \lambda\right)\left(\lambda-\bar{\lambda}_{k}\right)}=\frac{1}{2 \pi i} \oint \frac{d \lambda}{-\lambda_{j}\left(\lambda-1 / \lambda_{j}\right)\left(\lambda-\bar{\lambda}_{k}\right)}=-\frac{1}{1-\lambda_{j} \bar{\lambda}_{k}}
$$

that the matrix $\mathrm{G}=\left(\frac{\left(u_{j}, u_{k}\right) \mathcal{H}}{1-\lambda_{j} \bar{\lambda}_{k}}\right)_{j, k=1}^{n}$ differs in sign from the Gram matrix for functions $f_{j}(\lambda), j=1, \ldots, n$.

Proposition 3.7 Let $V$ be an isometric operator in a Pontryagin space $\mathfrak{H}$ and let $(\mathfrak{L}, \Gamma)$ be a unitary boundary pair for $V$. Then: 
(i) the set $\left(\sigma_{p}\left(V_{2}\right) \backslash \sigma_{p}(V)\right) \cap \mathbb{D}_{e}$ consists of at most $\kappa$ points and the corresponding eigenspaces are negative;

(ii) the set $\left(\sigma_{p}\left(V_{1}\right) \backslash \sigma_{p}(V)\right) \cap \mathbb{D}$ consists of at most $\kappa$ points and the corresponding eigenspaces are negative.

Proof (i) A point $\lambda \in \mathbb{D}_{e}$ belongs to the set $\sigma_{p}\left(V_{2}\right)$ if and only if there exists $\widehat{f_{\lambda}} \in$ $\widehat{\mathfrak{N}}_{\lambda}\left(V_{*}\right), f_{\lambda} \neq 0$, such that $\left\{\widehat{f_{\lambda}},\left(\begin{array}{c}u_{1} \\ 0\end{array}\right)\right\} \in \Gamma$ for some $u_{1} \in \mathfrak{L}_{1}$. The assumption $\lambda \notin \sigma_{p}(V)$ means that $u_{1} \neq 0$.

Now assume that $\lambda_{1}, \ldots, \lambda_{\kappa+1} \in \mathbb{D}_{e}$ and for some linearly independent vectors $\widehat{f}_{\lambda_{j}} \in \widehat{\mathfrak{N}}_{\lambda_{j}}\left(V_{*}\right) \backslash\{0\}$ and some $u_{1, j} \in \mathfrak{L}_{1} \backslash\{0\}$,

$$
\left\{\widehat{f}_{\lambda_{j}},\left(\begin{array}{c}
u_{1, j} \\
0
\end{array}\right)\right\} \in \Gamma, \quad j=1, \ldots, \kappa+1 .
$$

Then from (3.9) one gets

$$
\left[f_{\lambda_{j}}, f_{\lambda_{k}}\right]_{\mathfrak{H}}=\frac{\left(u_{1, j}, u_{1, k}\right)_{\mathfrak{L}_{1}}}{1-\lambda_{j} \bar{\lambda}_{k}}, \quad j, k=1, \ldots, \kappa+1
$$

If $\lambda_{j}=\lambda_{k}$ for some $j \neq k$ then the vectors $u_{j}$ and $u_{k}$ are linearly independent by the assumptions $\lambda_{j} \notin \sigma_{p}(V)$. On the other hand, if $\lambda_{j} \neq \lambda_{k}$, then the vector functions $f_{j}(\lambda)$ and $f_{k}(\lambda)$ defined in Lemma 3.6 are also linearly independent. Hence the matrix $G$ in Lemma 3.6 is invertible. One concludes that the form

$$
\sum_{j, k=1}^{\kappa+1}\left[f_{\lambda_{j}}, f_{\lambda_{k}}\right]_{\mathfrak{H}} \xi_{j} \bar{\xi}_{k}=\left[\sum_{j=1}^{\kappa+1} \xi_{j} f_{\lambda_{j}}, \sum_{k=1}^{\kappa+1} \xi_{k} f_{\lambda_{k}}\right]_{\mathfrak{H}}
$$

is negative for linearly independent vectors $\widehat{f}_{\lambda_{j}}$ when $\lambda_{j} \in \mathbb{D}_{e}$. This contradicts the assumption that the Pontryagin space $\mathfrak{H}$ has negative index $\kappa$.

(ii) The second statement is proved analogously.

In the sequel the following two subsets of $\mathbb{D}$ and $\mathbb{D}_{e}$ (cf. (2.10)) will often appear:

$$
\mathcal{D}:=\mathbb{D} \backslash \sigma_{p}\left(V_{1}\right), \quad \mathcal{D}_{e}:=\mathbb{D}_{e} \backslash \sigma_{p}\left(V_{2}\right)
$$

It should be noted that for various realization results and for the study of proper extensions of the isometry $V$ it is typically sufficient to assume that $\sigma_{p}(V)=\varnothing$; this is the case in particular when $V$ is a simple isometric operator in $\mathfrak{H}$. In this case Proposition 3.7 shows that both of the sets $\sigma_{p}\left(V_{2}\right) \cap \mathbb{D}_{e}$ and $\sigma_{p}\left(V_{1}\right) \cap \mathbb{D}$ contain at most $\kappa$ points.

Now consider the restrictions of (the graphs of) $\Gamma_{1}$ and $\Gamma_{2}$ to $\widehat{\mathfrak{N}}_{\lambda}\left(V_{*}\right)$,

$$
\Gamma_{1} \uparrow \widehat{\mathfrak{N}}_{\lambda}\left(V_{*}\right):=\left\{\left\{\widehat{f},\left(\begin{array}{c}
u_{1} \\
0
\end{array}\right)\right\} \in \Gamma_{1}: \widehat{f} \in \widehat{\mathfrak{N}}_{\lambda}\left(V_{*}\right), u_{1} \in \mathfrak{L}_{1}\right\}
$$


and

$$
\Gamma_{2} \uparrow \widehat{\mathfrak{N}}_{\lambda}\left(V_{*}\right):=\left\{\left\{\widehat{f},\left(\begin{array}{c}
0 \\
u_{2}
\end{array}\right)\right\} \in \Gamma_{2}: \widehat{f} \in \widehat{\mathfrak{N}}_{\lambda}\left(V_{*}\right), u_{2} \in \mathfrak{L}_{2}\right\} .
$$

It follows from (3.10) that

$$
\operatorname{ker}\left(\Gamma_{1}\left\lceil\widehat{\mathfrak{N}}_{\lambda}\left(V_{*}\right)\right)=\{0\} \quad(\lambda \in \mathcal{D}) ; \quad \operatorname{ker}\left(\Gamma_{2}\left\lceil\widehat{\mathfrak{N}}_{\lambda}\left(V_{*}\right)\right)=\{0\} \quad\left(\lambda \in \mathcal{D}_{e}\right)\right.\right.
$$

and the assumption $\sigma_{p}(V)=\emptyset$ guarantees in particular that the inverses

$$
\widehat{\gamma}_{1}(\lambda)=\left(\Gamma_{1}\left\lceil\widehat{\mathfrak{N}}_{\lambda}\left(V_{*}\right)\right)^{-1}(\lambda \in \mathcal{D}) ; \quad \widehat{\gamma}_{2}(\lambda)=\left(\Gamma_{2}\left\lceil\widehat{\mathfrak{N}}_{\lambda}\left(V_{*}\right)\right)^{-1}\left(\lambda \in \mathcal{D}_{e}\right)\right.\right.
$$

determine single-valued operator functions, which will be denoted by the same symbols

$$
\widehat{\gamma}_{1}(\lambda): \mathfrak{L}_{1} \rightarrow \widehat{\mathfrak{N}}_{\lambda}\left(V_{*}\right) \quad(\lambda \in \mathcal{D}) ; \quad \widehat{\gamma}_{2}(\lambda): \mathfrak{L}_{2} \rightarrow \widehat{\mathfrak{N}}_{\lambda}\left(V_{*}\right) \quad\left(\lambda \in \mathcal{D}_{e}\right) .
$$

Let $\pi_{1}$ and $\pi_{2}$ be projections onto $\mathfrak{L}_{1}$ and $\mathfrak{L}_{2}$ in $\mathfrak{L}$, respectively.

Definition 3.8 The operator functions

$$
\gamma_{1}(\lambda)=\pi_{1} \widehat{\gamma}_{1}(\lambda) \quad(\lambda \in \mathcal{D}) \quad \text { and } \quad \gamma_{2}(\lambda)=\pi_{1}{\widehat{\gamma_{2}}}_{(\lambda)}\left(\lambda \in \mathcal{D}_{e}\right)
$$

will be called the $\gamma$-fields of the unitary boundary pair $(\mathfrak{L}, \Gamma)$.

The definition of the $\gamma$-fields of the unitary boundary pair $(\mathfrak{L}, \Gamma)$ yields the following explicit formulas:

$$
\begin{aligned}
& \left\{\left(\begin{array}{c}
f_{\lambda} \\
\lambda f_{\lambda}
\end{array}\right),\left(\begin{array}{c}
u_{1} \\
u_{2}
\end{array}\right)\right\} \in \Gamma, \quad \widehat{f_{\lambda}} \in \widehat{\mathfrak{N}}_{\lambda}\left(V_{*}\right), \quad \lambda \in \mathcal{D} \quad \Longrightarrow \quad \gamma_{1}(\lambda) u_{1}=f_{\lambda} \\
& \left\{\left(\begin{array}{c}
f_{\lambda} \\
\lambda f_{\lambda}
\end{array}\right),\left(\begin{array}{c}
u_{1} \\
u_{2}
\end{array}\right)\right\} \in \Gamma, \quad \widehat{f_{\lambda}} \in \widehat{\mathfrak{N}}_{\lambda}\left(V_{*}\right), \quad \lambda \in \mathcal{D}_{e} \Longrightarrow \gamma_{2}(\lambda) u_{2}=f_{\lambda} .
\end{aligned}
$$

Later it is shown that $\gamma_{1}(\lambda)$ and $\gamma_{2}(\lambda)$ are bounded everywhere defined operators and holomorphic in $\lambda$; see Theorem 3.15.

Definition 3.9 The family of linear relations defined by

$$
\Theta(\lambda)=\left\{\left(\begin{array}{l}
u_{1} \\
u_{2}
\end{array}\right):\left\{\widehat{f_{\lambda}},\left(\begin{array}{l}
u_{1} \\
u_{2}
\end{array}\right)\right\} \in \Gamma, \widehat{f_{\lambda}} \in \widehat{\mathfrak{N}}_{\lambda}\left(V_{*}\right)\right\}, \quad \lambda \in \mathcal{D},
$$

will be called the Weyl family of $V$ corresponding to the unitary boundary pair $(\mathfrak{L}, \Gamma)$, or, briefly, the Weyl family of the unitary boundary pair $(\mathfrak{L}, \Gamma)$. 
In Theorem 3.15 it will be shown that the formula (3.15) determines a singlevalued operator function, which is called the Weyl function of $V$ corresponding to the boundary triple $(\mathfrak{L}, \Gamma)$. If the mapping $\Gamma$ is single-valued, then the Weyl function $\Theta(\lambda)$ can be defined by using the $\gamma$-fields

$$
\Theta(\lambda)=\Gamma_{2} \widehat{\gamma_{1}}(\lambda), \quad \lambda \in \mathcal{D}
$$

\subsection{Unitary Boundary Pairs and Unitary Colligations}

In the present section we consider a unitary boundary pair whose main transform is a unitary colligation

$$
\Delta=\left(\mathfrak{H}, \mathfrak{L}_{1}, \mathfrak{L}_{2}, U\right), \quad U=\left(\begin{array}{cc}
T & F \\
G & H
\end{array}\right):\left(\begin{array}{c}
\mathfrak{H} \\
\mathfrak{L}_{1}
\end{array}\right) \rightarrow\left(\begin{array}{c}
\mathfrak{H} \\
\mathfrak{L}_{2}
\end{array}\right)
$$

and write explicit formulas for all the objects connected with this unitary boundary pair in terms of the blocks of $U$.

Theorem 3.10 Let $\Delta=\left(\mathfrak{H}, \mathfrak{L}_{1}, \mathfrak{L}_{2}, U\right)$ be a unitary colligation of the form (3.17), let $\mathcal{U}=\operatorname{gr} U^{[*]}$, let $\Gamma=\mathcal{J}^{-1}(\mathcal{U})$ be a unitary relation from $\left(\mathfrak{H}^{2}, J_{\mathfrak{H}^{2}}\right)$ to $\left(\mathfrak{L}, J_{\mathfrak{L}}\right)$, i.e. $\mathcal{U}=\mathcal{J}(\Gamma)$ as in Lemma 3.3, and let

$$
V:=\operatorname{ker} \Gamma, \quad V_{*}:=\operatorname{dom} \Gamma, \quad V_{1}:=\operatorname{ker} \Gamma_{1}, \quad V_{2}:=\operatorname{ker} \Gamma_{2} .
$$

Then the following statements hold:

(i) The pair $(\mathfrak{L}, \Gamma)$ is a unitary boundary pair for $V$.

(ii) The unitary relation $\Gamma$ admits the representations

$$
\begin{aligned}
\Gamma & =\left\{\left\{\left(\begin{array}{c}
T h+F u_{1} \\
h
\end{array}\right),\left(\begin{array}{c}
u_{1} \\
G h+H u_{1}
\end{array}\right)\right\}: \begin{array}{c}
h \in \mathfrak{H} \\
u_{1} \in \mathfrak{L}_{1}
\end{array}\right\} \\
& =\left\{\left\{\left(\begin{array}{c}
g \\
T^{[*]} g+G^{[*]} u_{2}
\end{array}\right),\left(\begin{array}{c}
F^{[*]} g+H^{*} u_{2} \\
u_{2}
\end{array}\right)\right\}: \begin{array}{c}
g \in \mathfrak{H} \\
u_{2} \in \mathfrak{L}_{2}
\end{array}\right\} .
\end{aligned}
$$

(iii) The isometry $V$ in $\left(\mathfrak{H}, J_{\mathfrak{H}}\right)$ admits the representations

$$
V^{-1}=T \nmid \operatorname{ker} G, \quad V=T^{[*]} \uparrow \operatorname{ker} F^{[*]}
$$

and the linear relation $V_{*}$ takes the form

$$
V_{*}=\left\{\left(\begin{array}{c}
T h+F u_{1} \\
h
\end{array}\right): \begin{array}{c}
h \in \mathfrak{H} \\
u_{1} \in \mathfrak{L}_{1}
\end{array}\right\}=\left\{\left(\begin{array}{c}
g \\
T^{[*]} g+G^{[*]} u_{2}
\end{array}\right): \begin{array}{c}
g \in \mathfrak{H} \\
u_{2} \in \mathfrak{L}_{2}
\end{array}\right\} .
$$


(iv) The multivalued part of $\Gamma$ has the representations

$$
\operatorname{mul} \Gamma=\left\{\left(\begin{array}{c}
u_{1} \\
H u_{1}
\end{array}\right): u_{1} \in \operatorname{ker} F\right\}=\left\{\left(\begin{array}{c}
H^{*} u_{2} \\
u_{2}
\end{array}\right): v \in \operatorname{ker} G^{[*]}\right\}
$$

(v) The linear relations $V_{1}$ and $V_{2}$ are given by

$$
V_{1}=\left\{\left(\begin{array}{c}
T h \\
h
\end{array}\right): h \in \mathfrak{H}\right\}, \quad V_{2}=\left\{\left(\begin{array}{c}
g \\
T^{[*]} g
\end{array}\right): g \in \mathfrak{H}\right\},
$$

and hence the sets $\mathcal{D}:=\rho\left(V_{1}\right) \cap \mathbb{D}$ and $\mathcal{D}_{e}:=\rho\left(V_{2}\right) \cap \mathbb{D}_{e}$ are nonempty, they coincide with the sets in (3.10) and are connected by

$$
\mathcal{D}_{e}=\mathcal{D}^{\circ}:=\left\{\lambda \in \mathbb{D}_{e}: 1 / \bar{\lambda} \in \mathcal{D}\right\}
$$

moreover, card $(\mathbb{D} \backslash \mathcal{D})=\operatorname{card}\left(\mathbb{D}_{e} \backslash \mathcal{D}_{e}\right) \leq \kappa$

Proof (i) By Lemma $3.3 \Gamma=\mathcal{J}^{-1} \mathcal{U}$ is a unitary relation from $\left(\mathfrak{H}^{2}, J_{\mathfrak{H}^{2}}\right)$ to $\left(\mathfrak{L}, J_{\mathfrak{L}}\right)$. This means that the assumptions (1), (2) of Definition 3.1 are satisfied and, therefore, $(\mathfrak{L}, \Gamma)$ is a unitary boundary pair for the isometry $V=\operatorname{ker} \Gamma$ (which is an operator in view of (3) below).

(ii) Since the operator $U:\left(\begin{array}{c}\mathfrak{H} \\ \mathfrak{L}_{1}\end{array}\right) \rightarrow\left(\begin{array}{c}\mathfrak{H} \\ \mathfrak{L}_{2}\end{array}\right)$ is unitary then $\mathcal{U}=\operatorname{gr} U^{[*]}$ is also the graph of the operator $U^{-1}$ in (3.17), and hence $\mathcal{U}$ has the following representations

$$
\mathcal{U}=\operatorname{gr} U^{-1}=\left\{\left\{\left(\begin{array}{c}
T h+F u_{1} \\
G h+H u_{1}
\end{array}\right),\left(\begin{array}{c}
h \\
u_{1}
\end{array}\right)\right\}: \begin{array}{c}
h \in \mathfrak{H} \\
u_{1} \in \mathfrak{L}_{1}
\end{array}\right\} .
$$

In view of (3.6) this yields the first formula in (3.18). The equality

$$
\mathcal{U}=\operatorname{gr} U^{[*]}=\left\{\left\{\left(\begin{array}{c}
g \\
u_{2}
\end{array}\right),\left(\begin{array}{l}
T^{[*]} g+G^{[*]} u_{2} \\
F^{[*]} g+H^{[*]} u_{2}
\end{array}\right)\right\}: \begin{array}{r}
g \in \mathfrak{H} \\
u_{2} \in \mathfrak{L}_{2}
\end{array}\right\} .
$$

leads to the second representation of $\Gamma$ in (3.18).

(iii) and (iv) The formulas (3.19), (3.20), and (3.21) are all implied by (3.18).

(v) The formulas (3.22) for $V_{1}=\operatorname{ker} \Gamma_{1}$ and $V_{2}=\operatorname{ker} \Gamma_{2}$ are again obtained from (3.18). In particular, $V_{2}$ is the graph of the bounded operator $T^{[*]}$. It is closed and $\mathcal{D}_{e}=\rho\left(V_{2}\right)=\rho\left(T^{[*]}\right) \neq \emptyset$. Since $T^{[*]}$ is a contractive operator in the Pontryagin space $\left(\mathfrak{H}, J_{\mathfrak{H}}\right)$, its spectrum in $\mathbb{D}_{e}$ consists of at most $\kappa$ eigenvalues, so

$$
\operatorname{card}\left(\mathbb{D}_{e} \backslash \mathcal{D}_{e}\right)=\operatorname{card}\left(\mathbb{D}_{e} \backslash \rho\left(T^{[*]}\right)\right) \leq \kappa,
$$

see e.g. [31, p.91 Lemma 11.8]. Similarly, $V_{1}^{-1}$ is the graph of the contractive operator $T$ in the Pontryagin space $\left(\mathfrak{H}, J_{\mathfrak{H}}\right)$. Therefore,

$$
\mathcal{D}=\rho\left(V_{1}\right) \cap \mathbb{D}=\rho\left(T^{-1}\right) \cap \mathbb{D}=\left(\rho\left(T^{[*]}\right) \cap \mathbb{D}_{e}\right)^{\circ}=\mathcal{D}_{e}^{\circ} .
$$


This completes the proof.

By Proposition 3.2 the closure of $V_{*}$ is $V^{-[*]}$. Hence, by taking closures in (3.20) one arrives at the following representations for $V^{-[*]}$ :

$$
V^{-[*]}=\left\{\left(\begin{array}{c}
T h+f \\
h
\end{array}\right): \begin{array}{c}
h \in \mathfrak{H} \\
f \in \mathfrak{H}[-] \operatorname{ker} F^{[*]}
\end{array}\right\}
$$

and

$$
V^{-[*]}=\left\{\left(\begin{array}{c}
g \\
T^{[*]} g+f
\end{array}\right): \begin{array}{l}
g \in \mathfrak{H} \\
f \in \mathfrak{H}[-] \operatorname{ker} G
\end{array}\right\} .
$$

Theorem 3.11 Let $\Delta=\left(\mathfrak{H}, \mathfrak{L}_{1}, \mathfrak{L}_{2}, U\right)$ be a unitary colligation of the form (3.17), let $\mathcal{U}=\operatorname{gr} U^{[*]}$, let $\Gamma=\mathcal{J}^{-1} U$ and let the corresponding Weyl function $\Theta(\lambda)$ of $V$ and the $\gamma$-fields $\gamma_{1}(\lambda)$ and $\gamma_{2}(\lambda)$ be given by (3.15) and (3.13), (3.14). Then the following statements hold:

(i) The defect subspace $\mathfrak{N}_{\lambda}\left(V_{*}\right)$ takes the form

$$
\begin{aligned}
& \mathfrak{N}_{\lambda}\left(V_{*}\right)=\left\{(I-\lambda T)^{-1} F u_{1}: u_{1} \in \mathfrak{L}_{1}\right\}, \quad \lambda \in \mathcal{D} ; \\
& \mathfrak{N}_{\lambda}\left(V_{*}\right)=\left\{\left(\lambda I-T^{[*]}\right)^{-1} G^{[*]} u_{2}: u_{2} \in \mathfrak{L}_{2}\right\}, \quad \lambda \in \mathcal{D}_{e} .
\end{aligned}
$$

(ii) $\Gamma_{1}\left(\widehat{\mathfrak{N}}_{\lambda}\left(V_{*}\right)\right)=\mathfrak{L}_{1}, \lambda \in \mathcal{D}$, and $\Gamma_{2}\left(\widehat{\mathfrak{N}}_{\lambda}\left(V_{*}\right)\right)=\mathfrak{L}_{2}, \lambda \in \mathcal{D}_{e}$, and the $\gamma$-fields $\gamma_{1}(\lambda)$ and $\gamma_{2}(\lambda)$ take the form

$$
\begin{aligned}
& \gamma_{1}(\lambda)=(I-\lambda T)^{-1} F, \quad \lambda \in \mathcal{D} ; \\
& \gamma_{2}(\lambda)=\left(\lambda I-T^{[*]}\right)^{-1} G^{[*]}, \quad \lambda \in \mathcal{D}_{e} .
\end{aligned}
$$

(iii) The Weyl function $\Theta(\lambda)$ of $V$ corresponding to the unitary boundary pair $(\mathfrak{L}, \Gamma)$ coincides with the characteristic function of the colligation $\Delta$, i.e.,

$$
\Theta(\lambda)=\Theta_{\Delta}(\lambda)=H+\lambda G(I-\lambda T)^{-1} F, \quad \lambda \in \mathcal{D} .
$$

(iv) The unitary colligation $\Delta$ is closely connected if and only if the operator $V$ is simple.

Proof (i) Recall that $\widehat{\mathfrak{N}}_{\lambda}\left(V_{*}\right)$ consists of vectors $\left(\begin{array}{c}f_{\lambda} \\ \lambda f_{\lambda}\end{array}\right) \in V_{*}$. Therefore, the vector $\left(\begin{array}{c}T h_{\lambda}+F u_{1} \\ h_{\lambda}\end{array}\right)\left(h_{\lambda} \in \mathfrak{H}, u_{1} \in \mathfrak{L}_{1}\right)$ belongs to $\widehat{\mathfrak{N}}_{\lambda}\left(V_{*}\right)$ precisely when

$$
h_{\lambda}=\lambda\left(T h_{\lambda}+F u_{1}\right)
$$

Hence, for $\lambda \in \mathcal{D}=\rho\left(T^{-1}\right) \cap \mathbb{D}$ one obtains

$$
h_{\lambda}=\lambda(I-\lambda T)^{-1} F u_{1}, \quad \lambda \in \mathcal{D} .
$$


Similarly, the vector $\left(\begin{array}{c}g_{\lambda} \\ T^{[*]} g_{\lambda}+G^{[*]} u_{2}\end{array}\right) g_{\lambda} \in \mathfrak{H}, u_{2} \in \mathfrak{L}_{2}$ belongs to $\widehat{\mathfrak{N}}_{\lambda}\left(V_{*}\right)$ if and only if

$$
T^{[*]} g_{\lambda}+G^{[*]} u_{2}=\lambda g_{\lambda}
$$

Hence, for $\lambda \in \mathcal{D}_{e}=\rho\left(T^{[*]}\right) \cap \mathbb{D}_{e}$ one obtains

$$
g_{\lambda}=\left(\lambda I-T^{[*]}\right)^{-1} G^{[*]} u_{2}, \quad \lambda \in \mathcal{D}_{e} .
$$

(ii) By the first formula in (3.18) one gets

$$
\left\{\left(\begin{array}{c}
f_{\lambda} \\
\lambda f_{\lambda}
\end{array}\right),\left(\begin{array}{c}
u_{1} \\
H u_{1}+\lambda G(I-\lambda T)^{-1} F u_{1}
\end{array}\right)\right\} \in \Gamma, \quad \lambda \in \mathcal{D}
$$

and in view of (3.11) and (3.12) the $\gamma$-field $\gamma_{1}(\lambda)$ takes the form

$$
\gamma_{1}(\lambda) u_{1}=f_{\lambda}=(I-\lambda T)^{-1} F u_{1}, \quad \lambda \in \mathcal{D} .
$$

Similarly, by the second formula in (3.18)

$$
\left\{\left(\begin{array}{c}
g_{\lambda} \\
\lambda g_{\lambda}
\end{array}\right),\left(\begin{array}{c}
H^{*} u_{2}+F^{[*]}\left(\lambda I-T^{[*]}\right)^{-1} G^{[*]} u_{2} \\
u_{2}
\end{array}\right)\right\} \in \Gamma, \quad \lambda \in \mathcal{D}_{e}
$$

and hence

$$
\gamma_{2}(\lambda) u_{2}=g_{\lambda}=\left(\lambda I-T^{[*]}\right)^{-1} G^{[*]} u_{2}, \quad \lambda \in \mathcal{D}_{e} .
$$

(iii) It follows also from (3.28) that

$$
\Theta(\lambda) u_{1}=\left(H+\lambda G(I-\lambda T)^{-1} F\right) u_{1}=\Theta_{\Delta}(\lambda) u_{1}, \quad \lambda \in \mathcal{D}, u_{1} \in \mathfrak{L}_{1} .
$$

(iv) Notice that in view of (3.24) and (3.25) the defect subspaces $\mathfrak{N}_{\lambda}$ take the form

$$
\begin{array}{ll}
\mathfrak{N}_{\lambda}=\left\{(I-\lambda T)^{-1} f: f \in \mathfrak{H}[-] \operatorname{ker} F^{[*]}\right\}, & \lambda \in \mathcal{D} ; \\
\mathfrak{N}_{\lambda}=\left\{\left(\lambda I-T^{[*]}\right)^{-1} g: g \in \mathfrak{H}[-] \operatorname{ker} G\right\}, & \lambda \in \mathcal{D}_{e} .
\end{array}
$$

Comparison of (3.26), (3.27) with (3.30), (3.31) shows that the subspaces $\mathfrak{N}_{\lambda}\left(V_{*}\right)$ are dense in $\mathfrak{N}_{\lambda}$ for all $\lambda \in \mathcal{D} \cup \mathcal{D}_{e}$. Therefore, the set

$$
\overline{\operatorname{span}}\left\{\mathfrak{N}_{\lambda}: \lambda \in \mathcal{D}\right\} \quad\left(\text { resp. } \overline{\operatorname{span}}\left\{\mathfrak{N}_{\lambda}: \lambda \in \mathcal{D}_{e}\right\}\right)
$$

coincides with the set

$$
\overline{\operatorname{span}}\left\{\mathfrak{N}_{\lambda}\left(V_{*}\right): \lambda \in \mathcal{D}\right\} \quad\left(\text { resp. } \overline{\operatorname{span}}\left\{\mathfrak{N}_{\lambda}\left(V_{*}\right): \lambda \in \mathcal{D}_{e}\right\}\right)
$$


In view of the formulas (3.26), (3.27) one obtains the equalities

$$
\begin{aligned}
& \overline{\operatorname{span}}\left\{\mathfrak{N}_{\lambda}: \lambda \in \mathcal{D}\right\}=\overline{\operatorname{span}}\left\{\operatorname{ran}\left(T^{m} F\right): m \geq 0\right\}, \\
& \left.\overline{\operatorname{span}}\left\{\mathfrak{N}_{\lambda}: \lambda \in \mathcal{D}_{e}\right\}=\overline{\operatorname{span}}\left\{\operatorname{ran}\left(T^{[*]}\right)^{n} G^{[*]}\right): n \geq 0\right\} .
\end{aligned}
$$

By Definition 2.3 and the definition in (2.14) this proves the statement (iv).

Remark 3.12 With the assumptions of Theorem 3.11 there exist uniform limits

$$
\left(\gamma_{2}(\infty):=\right) \lim _{\lambda \rightarrow \infty} \gamma_{2}(\lambda)=0, \quad \lim _{\lambda \rightarrow \infty} \lambda \gamma_{2}(\lambda)=G^{[*]}
$$

\subsection{General Case}

To prove the desired statements for a general unitary boundary pair some preparatory lemmas will be used.

Let $\alpha \in \mathbb{C}$ such that $|\alpha| \neq 1$ and define the transform $M^{(\alpha)} \in \mathbf{B}\left(\mathfrak{H}^{2}\right)$ in the space $\left(\mathfrak{H}^{2}, J_{\mathfrak{H}^{2}}\right)$ by

$$
M^{(\alpha)}=\frac{1}{\beta}\left(\begin{array}{cc}
I & -\alpha I \\
-\bar{\alpha} I & I
\end{array}\right), \quad \beta:=\sqrt{1-|\alpha|^{2}} .
$$

It is easy to check that $M^{(\alpha)}$ is a standard unitary operator in the Krel̆n space $\left(\mathfrak{H}^{2}, J_{\mathfrak{H}^{2}}\right)$. Clearly, $M^{(0)}=I$ and

$$
\left(M^{(\alpha)}\right)^{-1}=M^{(-\alpha)}, \quad|\alpha| \neq 1
$$

Associated with $M^{(\alpha)}$ define a transform of the extended complex plane $\mathbb{C} \cup\{\infty\}$ by the formula

$$
\mu^{(\alpha)}(\lambda):=\frac{\lambda-\bar{\alpha}}{1-\alpha \lambda} .
$$

Lemma 3.13 The transform $M^{(\alpha)}$ in (3.35) maps closed subspaces of the Kre rn space $\left(\mathfrak{H}^{2}, J_{\mathfrak{H}^{2}}\right)$ back to closed subspaces and it satisfies

$$
\left(M^{(\alpha)}\left(\mathcal{S}^{-1}\right)\right)=\left(M^{(\bar{\alpha})}(\mathcal{S})\right)^{-1}
$$

and

$$
M^{(\alpha)}\left(\mathcal{S}^{-[*]}\right)=\left(M^{(\alpha)}(\mathcal{S})\right)^{-[*]}
$$

In particular, $M^{(\alpha)}$ maps isometric (unitary, contractive, expanding) relations $\mathcal{S}$ in $\mathfrak{H}$ back to isometric (unitary, contractive, expanding) relations $M^{(\alpha)}(\mathcal{S})$ in $\mathfrak{H}$ and, moreover, for any closed linear relation $\mathcal{S}$ in $\mathfrak{H}$ the following statements hold with $1-\alpha \lambda \neq 0$ : 
(i) $\operatorname{ker}(\mathcal{S}-\lambda)=\operatorname{ker}\left(M^{(\alpha)}(\mathcal{S})-\mu^{(\alpha)}(\lambda) I\right)$, mul $\mathcal{S}=\operatorname{ker}\left(\alpha M^{(\alpha)}(\mathcal{S})+I\right)$;

(ii) $\operatorname{ran}(\mathcal{S}-\lambda)=\operatorname{ran}\left(M^{(\alpha)}(\mathcal{S})-\mu^{(\alpha)}(\lambda) I\right), \operatorname{dom} \mathcal{S}=\operatorname{ran}\left(\alpha M^{(\alpha)}(\mathcal{S})+I\right)$;

(iii) $\lambda \in \sigma_{j}(\mathcal{S}) \Longleftrightarrow \mu^{(\alpha)}(\lambda) \in \sigma_{j}\left(M^{(\alpha)}(\mathcal{S})\right)$ for $j=p, c, r$;

(iv) $\lambda \in \rho(\mathcal{S}) \Longleftrightarrow \mu^{(\alpha)}(\lambda) \in \rho\left(M^{(\alpha)}(\mathcal{S})\right)$.

Proof The mapping $M^{(\alpha)}$ is unitary in the Krel̆n space $\left(\mathfrak{H}^{2}, J_{\mathfrak{H}^{2}}\right)$ and, in fact, also unitary and selfadjoint as a linear operator on the Hilbert space $\mathfrak{H}^{2}$. Therefore, it maps closed subsets to closed subsets in $\mathfrak{H}^{2}$. The formula (3.37) is checked with a straightforward calculation. Moreover, for all $\widehat{f}=\left\{f, f^{\prime}\right\}, \widehat{g}=\left\{g, g^{\prime}\right\}$

$$
\left(J_{\mathfrak{H}^{2}} \widehat{f}, \widehat{g}\right)=\left(J_{\mathfrak{H}^{2}} M^{(\alpha)} \widehat{f}, M^{(\alpha)} \widehat{g}\right)
$$

which shows that if $\mathcal{S}$ is isometric (contractive, expanding) relation in $\mathfrak{H}$ so is its image $M^{(\alpha)}(\mathcal{S})$, since the expression in (3.39) with $\widehat{f}=\widehat{g} \in \mathcal{S}$ takes the value $=0$ (resp. $\geq 0$ and $\leq 0$ ). Moreover, (3.39) implies the property (3.38).

The relation $\mathcal{S}$ in the Pontryagin space $\mathfrak{H}$ is unitary precisely when $S=S^{-[*]}$ and hence (3.39) implies that then also $M^{(\alpha)}(\mathcal{S})=\left(M^{(\alpha)}(\mathcal{S})\right)^{-[*]}$, i.e., $M^{(\alpha)}(\mathcal{S})$ is a unitary relation in $\mathfrak{H}$.

To prove the remaining assertions let $\left\{f, f^{\prime}\right\} \in \mathcal{S}$. Then $\left\{f, f^{\prime}-\lambda f\right\} \in(\mathcal{S}-\lambda I)$ and this is equivalent to

$$
\left(\begin{array}{c}
\frac{1}{\beta}\left(f-\alpha f^{\prime}\right) \\
\frac{\beta}{1-\alpha \lambda}\left(f^{\prime}-\lambda f\right)
\end{array}\right) \in M^{\alpha}(\mathcal{S})-\mu^{(\alpha)}(\lambda) I,
$$

where $\alpha \in \mathbb{D}, 1-\alpha \lambda \neq 0$. This formula with $\lambda \in \mathbb{C}$ gives the equalities for ker $(\mathcal{S}-\lambda)$ and $\operatorname{ran}(\mathcal{S}-\lambda)$. Analogously the choice $\lambda=\infty$ corresponds to $\mu^{(\alpha)}(\infty)=-1 / \alpha$ and this yields the formulas for mul $\mathcal{S}$ and $\operatorname{dom} \mathcal{S}$ in (i) and (ii).

The statements (iii) and (iv) follow from (i) and (ii) when applying the definitions of the resolvent set $\rho(\mathcal{S})$ and the spectral components $\sigma_{j}(\mathcal{S}), j=s, c, r$. pair.

In the next lemma the transform $M^{(\alpha)} \in \mathbf{B}\left(\mathfrak{H}^{2}\right)$ is composed with a unitary boundary

Lemma 3.14 Let $V$ be a closed isometric operator in a Pontryagin space $\mathfrak{H}$, let $(\mathfrak{L}, \Gamma)$ be a unitary boundary pair for $V$, and let $\alpha \in \mathbb{C},|\alpha| \neq 1$. Then

$$
V^{(\alpha)}:=M^{(\alpha)}(V)
$$

is also a closed isometric relation in $\mathfrak{H}$. Moreover, mul $V^{(\alpha)}=\{0\}$ precisely when $\alpha^{-1} \notin \sigma_{p}(V)$, and in this case:

(i) The composition

$$
\Gamma^{(\alpha)}:=\Gamma \circ M^{(-\alpha)}
$$

defines a unitary boundary pair $\left(\mathfrak{L}, \Gamma^{(\alpha)}\right)$ for the isometric operator $V^{(\alpha)}$. 
(ii) The Weyl function $\Theta^{(\alpha)}$ and the $\gamma$-fields $\gamma_{1}^{(\alpha)}, \gamma_{2}^{(\alpha)}$ of the unitary boundary pair $\left(\mathfrak{L}, \Gamma^{(\alpha)}\right)$ are connected to the Weyl function and the $\gamma$-fields of the unitary boundary pair $(\mathfrak{L}, \Gamma)$ by

$$
\begin{aligned}
& \Theta^{(\alpha)}(\mu(\alpha))=\Theta(\lambda), \quad \lambda \in \mathcal{D}, \\
& \gamma_{1}^{(\alpha)}(\mu(\alpha))=\frac{1-\lambda \alpha}{\beta} \gamma_{1}(\lambda), \quad \lambda \in \mathcal{D}, \\
& \gamma_{2}^{(\alpha)}(\mu(\alpha))=\frac{1-\lambda \alpha}{\beta} \gamma_{2}(\lambda), \quad \lambda \in \mathcal{D}_{e} .
\end{aligned}
$$

Proof The statements concerning the linear relation $V^{(\alpha)}$ are implied by Lemma 3.13.

(i) By definition $M^{(-\alpha)}$ is a standard unitary operator in the Krěn space $\left(\mathfrak{H}^{2}, J_{\mathfrak{H}^{2}}\right)$. Therefore, the composition $\Gamma^{(\alpha)}=\Gamma \circ M^{(-\alpha)}$ is a unitary relation from the Krĕn space $\left(\mathfrak{H}^{2}, J_{\mathfrak{H}^{2}}\right)$ to the Kreı̆n space $\left(\mathfrak{L}, J_{\mathfrak{L}}\right)$. It follows from the equivalence

$$
\{\widehat{f}, \widehat{u}\} \in \Gamma \Longleftrightarrow\left\{M^{(\alpha)} \widehat{f}, \widehat{u}\right\} \in \Gamma^{(\alpha)}
$$

that $\operatorname{ker} \Gamma^{(\alpha)}=M^{(\alpha)}(V)$ and $\operatorname{dom} \Gamma^{(\alpha)}=M^{(\alpha)}\left(V_{*}\right)$; see (3.36).

(ii) By Lemma 3.13

$$
\lambda \in \sigma_{p}\left(V_{*}\right) \Leftrightarrow \mu^{(\alpha)}(\lambda) \in \sigma_{p}\left(V_{*}^{(\alpha)}\right)
$$

and hence the defect subspaces $\mathfrak{N}_{\omega}\left(V_{*}^{(\alpha)}\right):=\operatorname{ker}\left(V_{*}^{(\alpha)}-\omega I\right)$ are connected with the defect subspaces $\mathfrak{N}_{\lambda}\left(V_{*}\right)$ by

$$
\mathfrak{N}_{\lambda}\left(V_{*}\right)=\mathfrak{N}_{\mu^{(\alpha)}(\lambda)}\left(V_{*}^{(\alpha)}\right) .
$$

Rewriting the equivalence (3.44) for vectors $f \in \mathfrak{N}_{\lambda}\left(V_{*}\right)$ we obtain

$$
\left\{\left(\begin{array}{c}
f \\
\lambda f
\end{array}\right),\left(\begin{array}{l}
u_{1} \\
u_{2}
\end{array}\right)\right\} \in \Gamma \Longleftrightarrow\left\{\frac{1-\lambda \alpha}{\beta}\left(\begin{array}{c}
f \\
\mu^{(\alpha)}(\lambda) f
\end{array}\right),\left(\begin{array}{l}
u_{1} \\
u_{2}
\end{array}\right)\right\} \in \Gamma^{(\alpha)} .
$$

In view of Definition 3.8 this implies

$$
\begin{array}{ll}
\gamma_{1}^{(\alpha)}\left(\mu^{(\alpha)}(\lambda)\right) u_{1}=\frac{1-\lambda \alpha}{\beta} \gamma_{1}(\lambda) u_{1}, & \lambda \in \mathcal{D} ; \\
\gamma_{2}^{(\alpha)}\left(\mu^{(\alpha)}(\lambda)\right) u_{2}=\frac{1-\lambda \alpha}{\beta} \gamma_{2}(\lambda) u_{2}, & \lambda \in \mathcal{D}_{e} .
\end{array}
$$

By virtue of (3.50) this proves (ii).

The next two theorems contain a full characterization of the class of Weyl functions $\Theta(\lambda)$ of boundary pairs. In the first theorem it is shown that $\Theta(\lambda)$ belongs to the generalized Schur class $\mathcal{S}_{\kappa}\left(\mathfrak{L}_{1}, \mathfrak{L}_{2}\right)$. 
Theorem 3.15 Let $(\mathfrak{L}, \Gamma)$ be a unitary boundary pair for an isometric operator $V$ in a Pontryagin space $\mathfrak{H}$ and let $V_{1}=\operatorname{ker} \Gamma_{1}, V_{2}=\operatorname{ker} \Gamma_{2}$. Then:

(i) $V_{1}$ and $V_{2}$ are closed linear relations which are connected by $V_{1}=V_{2}^{-[*]}$ and, moreover, the sets

$$
\mathcal{D}:=\rho\left(V_{1}\right) \cap \mathbb{D} \text { and } \mathcal{D}_{e}:=\rho\left(V_{2}\right) \cap \mathbb{D}_{e}
$$

coincide with the sets in (3.10) and they are connected by (3.23). In particular, they are nonempty and the sets $\mathbb{D} \backslash \mathcal{D}$ and $\mathbb{D}_{e} \backslash \mathcal{D}_{e}$ contain at most $\kappa$ points.

(ii) The $\gamma$-field $\gamma_{1}(\lambda)$ is holomorphic on $\mathcal{D}$ with values in $\mathbf{B}\left(\mathfrak{L}_{1}, \mathfrak{H}\right)$.

(iii) The $\gamma$-field $\gamma_{2}(\lambda)$ is holomorphic on $\mathcal{D}_{e}$ with values in $\mathbf{B}\left(\mathfrak{L}_{2}, \mathfrak{H}\right)$. If $0 \in \mathcal{D}$ and $\gamma_{2}^{\#}$ is defined by

$$
\gamma_{2}^{\#}(\lambda):=\gamma_{2}\left(\bar{\lambda}^{-1}\right)^{*}, \quad \lambda \in \mathcal{D}
$$

then the following uniform limits exist

$$
\left(\gamma_{2}^{\#}(0):=\right) \lim _{\lambda \rightarrow 0} \gamma_{2}^{\#}(\lambda)=0, \quad\left(\left(\gamma_{2}^{\#}\right)^{\prime}(0):=\right) \lim _{\lambda \rightarrow 0} \frac{1}{\lambda} \gamma_{2}^{\#}(\lambda)\left(\in \mathbf{B}\left(\mathfrak{H}, \mathfrak{L}_{2}\right)\right)
$$

(iv) The Weyl function $\Theta(\lambda)$ is holomorphic on $\mathcal{D}$, takes values in $\mathbf{B}\left(\mathfrak{L}_{1}, \mathfrak{L}_{2}\right)$ for $\lambda \in \mathcal{D}$ and belongs to the class $\mathcal{S}_{K}\left(\mathfrak{L}_{1}, \mathfrak{L}_{2}\right)$.

(v) For all $\lambda \in \mathcal{D}$ the following relation holds

$$
\left\{\left(\begin{array}{c}
\gamma_{1}(\lambda) u_{1} \\
\lambda \gamma_{1}(\lambda) u_{1}
\end{array}\right),\left(\begin{array}{c}
u_{1} \\
\Theta(\lambda) u_{1}
\end{array}\right)\right\} \in \Gamma, \quad u_{1} \in \mathfrak{L}_{1}, \lambda \in \mathcal{D}
$$

and for all $\lambda \in \mathcal{D}_{e}$ the following relation holds

$$
\left\{\left(\begin{array}{c}
\gamma_{2}(\lambda) u_{2} \\
\lambda \gamma_{2}(\lambda) u_{2}
\end{array}\right),\left(\begin{array}{c}
\Theta^{\#}(\lambda) u_{2} \\
u_{2}
\end{array}\right)\right\} \in \Gamma, \quad u_{2} \in \mathfrak{L}_{2}, \lambda \in \mathcal{D}_{e}
$$

where

$$
\Theta^{\#}(\lambda):=\Theta(1 / \bar{\lambda})^{*} \quad\left(\lambda \in \mathcal{D}_{e}\right)
$$

(vi) Moreover, the following equalities are satisfied

$$
\begin{array}{ll}
\gamma_{1}(\lambda)=\gamma_{1}(\mu)+(\lambda-\mu)\left(V_{1}-\lambda I\right)^{-1} \gamma_{1}(\mu), & \lambda, \mu \in \mathcal{D} ; \\
\gamma_{2}(\lambda)=\gamma_{2}(\mu)+(\lambda-\mu)\left(V_{2}-\lambda I\right)^{-1} \gamma_{2}(\mu), & \lambda, \mu \in \mathcal{D}_{e} .
\end{array}
$$

Proof (i) By Proposition 3.7 there is $\alpha \in \mathbb{D}$, such that

$$
\operatorname{ker}\left(I-\alpha V_{2}\right)=\{0\} \text {. }
$$


Then the linear relation $V^{(\alpha)}=M^{(\alpha)} V$ is isometric and single-valued. By Lemma 3.14 the pair $\left(\mathfrak{L}, \Gamma^{(\alpha)}\right)$ with $\Gamma^{(\alpha)}=\Gamma \circ M^{(-\alpha)}$ is a unitary boundary pair for the isometric operator $V^{(\alpha)}$.

The linear relations $V_{1}^{(\alpha)}:=\operatorname{ker} \Gamma_{1}^{(\alpha)}, V_{2}^{(\alpha)}:=\operatorname{ker} \Gamma_{2}^{(\alpha)}$ are related with the linear relations $V_{1}$ and $V_{2}$ by the equalities

$$
\begin{aligned}
& V_{1}^{(\alpha)}=M^{(\alpha)}\left(V_{1}\right)=\left\{\left(\begin{array}{c}
f-\alpha f^{\prime} \\
-\bar{\alpha} f+f^{\prime}
\end{array}\right):\left\{f, f^{\prime}\right\} \in V_{1}\right\}, \\
& V_{2}^{(\alpha)}=M^{(\alpha)}\left(V_{2}\right)=\left\{\left(\begin{array}{c}
f-\alpha f^{\prime} \\
-\bar{\alpha} f+f^{\prime}
\end{array}\right):\left\{f, f^{\prime}\right\} \in V_{2}\right\} .
\end{aligned}
$$

By the choice of $\alpha$ we have mul $V_{2}^{(\alpha)}=\{0\}$. Let $\mathcal{U}^{(\alpha)}=\mathcal{J} \Gamma^{(\alpha)}$. By Proposition 3.4 mul $\mathcal{U}^{(\alpha)}=\{0\}$, which by Lemma $2.5(\mathrm{v})$ implies that $\mathcal{U}^{(\alpha)}$ is (the graph of) a unitary colligation $U^{(\alpha)}$. Now an application of Theorem 3.10 shows that $V_{2}^{(\alpha)}$ and $\left(V_{1}^{(\alpha)}\right)^{-1}$ are graphs of closed bounded operators defined everywhere on $\mathfrak{H}$ and $V_{1}^{(\alpha)}=\left(V_{2}^{(\alpha)}\right)^{-[*]}$; see (3.22). In view of (3.55) and (3.56) the linear relations $V_{1}$ and $V_{2}$ are closed and (3.38) implies that $V_{1}=V_{2}^{-[*]}$; cf. Lemma 3.13. Moreover, it follows from Lemma 3.13 that with $j=1,2$,

$$
\lambda \in \rho\left(V_{j}\right) \Leftrightarrow \mu^{(\alpha)}(\lambda) \in \rho\left(V_{j}^{(\alpha)}\right) .
$$

Hence the set $\mathcal{D}:=\rho\left(V_{1}\right) \cap \mathbb{D}$ is nonempty and card $(\mathbb{D} \backslash \mathcal{D}) \leq \kappa$ since the same properties hold for the set $\mathcal{D}^{(\alpha)}:=\rho\left(V_{1}^{(\alpha)}\right) \cap \mathbb{D}$; see Theorem $3.10(\mathrm{v})$.

Similarly, by Theorem $3.10(\mathrm{v})$ the set $\mathcal{D}_{e}^{(\alpha)}:=\rho\left(V_{2}^{(\alpha)}\right) \cap \mathbb{D}_{e}$ is nonempty, card $\left(\mathbb{D}_{e} \backslash \mathcal{D}_{e}^{(\alpha)}\right) \leq \kappa$ and by (3.57) this implies the corresponding statement for $\mathbb{D}_{e} \backslash \mathcal{D}_{e}$.

(ii) and (iii) By Theorem 3.11 the $\gamma$-field $\gamma_{1}^{(\alpha)}(\lambda)$ is holomorphic on $\mathcal{D}^{(\alpha)}$ with values in $\mathbf{B}\left(\mathfrak{L}_{1}, \mathfrak{H}\right)$ and the $\gamma$-field $\gamma_{2}^{(\alpha)}(\lambda)$ is holomorphic on $\mathcal{D}_{e}^{(\alpha)}$ with values in $\mathbf{B}\left(\mathfrak{L}_{2}, \mathfrak{H}\right)$. The desired statement for the $\gamma$-fields $\gamma_{1}(\lambda)$ and $\gamma_{2}(\lambda)$ follows from (3.42) and (3.43).

The existence of the limits in (3.49) is obtained from (3.42) and Remark 3.12.

(iv) This statement is implied by Theorem 3.11 (iii) and (3.41).

(v) The relation (3.50) is implied by (3.14), (3.15) and items (ii), (iv).

To prove (3.51), let us choose for $\lambda \in \mathcal{D}_{e}, u_{2} \in \mathfrak{L}_{2}$, and a unique vector $v \in \mathfrak{L}_{1}$, such that

$$
\left\{\left(\begin{array}{c}
\gamma_{2}(\lambda) u_{2} \\
\lambda \gamma_{2}(\lambda) u_{2}
\end{array}\right),\left(\begin{array}{c}
v \\
u_{2}
\end{array}\right)\right\} \in \Gamma .
$$


By applying (3.2) or (3.9) to the elements (3.58) with $\lambda \in \mathcal{D}_{e}$ and (3.50) with $\lambda$ replaced by $\bar{\lambda}^{-1} \in \mathcal{D}$ it is seen that for all $u_{1} \in \mathfrak{L}_{1}$

$$
\begin{aligned}
0 & =\left[\gamma_{2}(\lambda) u_{2}, \gamma_{1}\left(\bar{\lambda}^{-1}\right) u_{1}\right]_{\mathfrak{H}}-\left[\lambda \gamma_{2}(\lambda) u_{2}, \bar{\lambda}^{-1} \gamma_{1}\left(\bar{\lambda}^{-1}\right) u_{1}\right]_{\mathfrak{H}} \\
& =\left(v, u_{1}\right) \mathfrak{L}_{1}-\left(u_{2}, \Theta\left(\bar{\lambda}^{-1}\right) u_{1}\right) \mathfrak{L}_{2}
\end{aligned}
$$

and hence $v=\Theta\left(\bar{\lambda}^{-1}\right)^{*} u_{2}=\Theta^{\#}(\lambda) u_{2}$.

(vi) To prove the identity (3.52) consider the vector $f_{\mu}=\gamma_{1}(\mu) u_{1} \in \mathfrak{N}_{\mu}\left(V_{*}\right)$, where $u_{1} \in \mathfrak{L}_{1}, \mu \in \mathcal{D}$. Then there exists $u_{2} \in \mathfrak{L}_{2}$ such that

$$
\left\{\left(\begin{array}{c}
f_{\mu} \\
\mu f_{\mu}
\end{array}\right),\left(\begin{array}{c}
u_{1} \\
u_{2}
\end{array}\right)\right\} \in \Gamma, \quad \mu \in \mathcal{D} .
$$

For $\lambda \in \mathcal{D}$ consider the vector

$$
\widehat{f_{\lambda}}=\left(\begin{array}{c}
f_{\mu} \\
\mu f_{\mu}
\end{array}\right)+\widehat{g}, \quad \text { where } \widehat{g}=(\lambda-\mu)\left(\begin{array}{c}
\left(V_{1}-\lambda I\right)^{-1} f_{\mu} \\
I+\lambda\left(V_{1}-\lambda I\right)^{-1} f_{\mu}
\end{array}\right) \in V_{1} \subset V_{*} .
$$

Direct calculations show that $\widehat{f_{\lambda}} \in \widehat{\mathfrak{N}}_{\lambda}\left(V_{*}\right)$. Since $\widehat{g}=\left(\begin{array}{c}g \\ g^{\prime}\end{array}\right) \in V_{1}$ there exists $v_{2} \in \mathfrak{L}_{2}$ such that

$$
\left\{\left(\begin{array}{c}
g \\
g^{\prime}
\end{array}\right),\left(\begin{array}{c}
0 \\
v_{2}
\end{array}\right)\right\} \in \Gamma \text {. }
$$

It follows from (3.59) and (3.60) that

$$
\left\{\left(\begin{array}{c}
f_{\lambda} \\
\lambda f_{\lambda}
\end{array}\right),\left(\begin{array}{c}
u_{1} \\
u_{2}+v_{2}
\end{array}\right)\right\}=\left\{\left(\begin{array}{c}
f_{\mu}+g \\
\mu f_{\mu}+g^{\prime}
\end{array}\right),\left(\begin{array}{c}
u_{1} \\
u_{2}+v_{2}
\end{array}\right)\right\} \in \Gamma
$$

and hence $\gamma_{1}(\lambda) u_{1}=f_{\lambda}$. This proves (3.52).

The equality (3.53) is proved similarly.

There is an analog for the notion of transposed boundary triple (see [19]) for boundary pairs of isometric operators. In the present case this notion contains the second boundary triple associated with a dual pair $\left\{V, V^{-1}\right\}$ as defined in [41] in the case of ordinary boundary triples for Hilbert space isometries. For this purpose the notion of transposed boundary pair $\left(\mathfrak{L}_{2} \oplus \mathfrak{L}_{1}, \Gamma^{\top}\right)$ is introduced for boundary pairs of isometric operators and its basic properties are established in the next proposition.

Proposition 3.16 Let $(\mathfrak{L}, \Gamma)$ be a unitary boundary pair for an isometric operator $V$ in a Pontryagin space $\mathfrak{H}$, let $V_{1}=\operatorname{ker} \Gamma_{1}, V_{2}=\operatorname{ker} \Gamma_{2}$, let $\gamma_{1}(\lambda)$ and $\gamma_{2}(\lambda)$ be the $\gamma$-fields of $(\mathfrak{L}, \Gamma)$, let $\Theta(\lambda)$ be the Weyl function of $(\mathfrak{L}, \Gamma)$, and define

$$
\Gamma^{\top}=\left\{\left\{\left(\begin{array}{l}
f^{\prime} \\
f
\end{array}\right),\left(\begin{array}{l}
u_{2} \\
u_{1}
\end{array}\right)\right\}:\left\{\left(\begin{array}{l}
f \\
f^{\prime}
\end{array}\right),\left(\begin{array}{l}
u_{1} \\
u_{2}
\end{array}\right)\right\} \in \Gamma\right\} .
$$


Then:

(i) $\left(\mathfrak{L}_{2} \oplus \mathfrak{L}_{1}, \Gamma^{\top}\right)$ is a unitary boundary pair for $V^{-1}$.

(ii) $V_{1}^{\top}:=\operatorname{ker} \Gamma_{1}^{\top}=V_{2}^{-1}$ and $V_{2}^{\top}:=\operatorname{ker} \Gamma_{2}^{\top}=V_{1}^{-1}$.

(iii) The Weyl function $\Theta^{\top}(\lambda)$ and the $\gamma$-fields $\gamma_{1}^{\top}(\lambda)$ and $\gamma_{2}^{\top}(\lambda)$, corresponding to the pair $\left(\mathfrak{L}_{2} \oplus \mathfrak{L}_{1}, \Gamma^{\top}\right)$ are connected with $\Theta(\lambda), \gamma_{1}(\lambda)$ and $\gamma_{2}(\lambda)$ by

$$
\begin{aligned}
& \Theta^{\top}(\lambda)=\Theta(\bar{\lambda})^{*}, \quad \lambda \in \overline{\mathcal{D}}:=\{\bar{\mu}: \mu \in \mathcal{D}\}, \\
& \gamma_{1}^{\top}(\lambda)=\frac{1}{\lambda} \gamma_{2}\left(\frac{1}{\lambda}\right), \quad \lambda \in \overline{\mathcal{D}} ; \quad \gamma_{2}^{\top}(\lambda)=\frac{1}{\lambda} \gamma_{1}\left(\frac{1}{\lambda}\right), \quad \lambda \in \overline{\mathcal{D}}_{e} .
\end{aligned}
$$

Proof (i) This statement is implied by the equality $V=\operatorname{ker} \Gamma$ and the following identity

$$
\left[f^{\prime}, g^{\prime}\right]_{\mathfrak{H}}-[f, g]_{\mathfrak{H}}=\left(u_{2}, v_{2}\right) \mathfrak{L}_{2}-\left(u_{1}, v_{1}\right) \mathfrak{L}_{1},
$$

which is valid for all $\{\widehat{f}, \widehat{u}\},\{\widehat{g}, \widehat{v}\} \in \Gamma$; see Definition 3.1.

(ii) The statement is clear from the definition of $\left(\mathfrak{L}_{2} \oplus \mathfrak{L}_{1}, \Gamma^{\top}\right)$.

(iii) If $\lambda \in \overline{\mathcal{D}}$ then $1 / \lambda \in \mathcal{D}_{e}$ and one obtains from (3.51)

$$
\left\{\left(\begin{array}{c}
\gamma_{2}(1 / \lambda) u_{2} \\
1 / \lambda \gamma_{2}(1 / \lambda) u_{2}
\end{array}\right),\left(\begin{array}{c}
\Theta^{\#}(1 / \lambda) u_{2} \\
u_{2}
\end{array}\right)\right\} \in \Gamma, \quad u_{2} \in \mathfrak{L}_{2}, \quad \lambda \in \overline{\mathcal{D}} \text {. }
$$

In view of (3.61) this implies

$$
\left\{\left(\begin{array}{c}
1 / \lambda \gamma_{2}(1 / \lambda) u_{2} \\
\gamma_{2}(1 / \lambda) u_{2}
\end{array}\right),\left(\begin{array}{c}
u_{2} \\
\Theta^{\#}(1 / \lambda) u_{2}
\end{array}\right)\right\} \in \Gamma^{\top}, \quad u_{2} \in \mathfrak{L}_{2}, \quad \lambda \in \overline{\mathcal{D}},
$$

and hence

$$
\gamma_{1}^{\top}(\lambda)=\frac{1}{\lambda} \gamma_{2}\left(\frac{1}{\lambda}\right) \quad \text { and } \quad \Theta^{\top}(\lambda)=\Theta^{\#}(1 / \lambda)=\Theta(\bar{\lambda})^{*}, \quad \lambda \in \overline{\mathcal{D}} .
$$

The second equality in (3.63) is proved similarly.

\subsection{Realization Theorem}

The converse statement to Theorem 3.15 contains the main realization result for the generalized Schur class $\mathcal{S}_{\kappa}\left(\mathfrak{L}_{1}, \mathfrak{L}_{2}\right)$ : every function $\Theta(\lambda)$ from the class $\mathcal{S}_{\kappa}\left(\mathfrak{L}_{1}, \mathfrak{L}_{2}\right)$ can be realized as the Weyl function of a boundary pair for some isometric operator $V$ in a Pontryagin space.

Theorem 3.17 Let $s(\cdot) \in \mathcal{S}_{\kappa}\left(\mathfrak{L}_{1}, \mathfrak{L}_{2}\right)$ with the domain of holomorphy $\mathfrak{h}_{s}(\subset \mathbb{D})$. Then there exists a simple isometric operator $V$ in a Pontryagin space $\mathfrak{H}$ and a unitary boundary pair $\left(\mathfrak{L}_{1} \times \mathfrak{L}_{2}, \Gamma\right)$ such that the corresponding Weyl function $\Theta(\lambda)$ coincides with $s(\lambda)$ on $\mathfrak{h}_{s}$. 
Proof (1) First assume that $s(\cdot)$ is holomorphic at 0 . Then by [1, Theorem 2.3.1] there exists a closely connected unitary colligation $U=\left(\begin{array}{ll}T & F \\ G & H\end{array}\right)$ such that its characteristic function coincides with $s(\lambda)$ for all $\lambda \in \mathfrak{h}_{s}$.

Let $V$ be defined by the formula $V=T^{[*]} \uparrow \operatorname{ker} F^{[*]}$ and let the linear relation $\Gamma$ be defined by (3.18). Then by Theorem 3.10 the pair $(\mathfrak{L}, \Gamma)$ is a unitary boundary pair for $V_{*}$ and the corresponding Weyl function $\Theta(\lambda)$ coincides with $s(\lambda)$ on $\mathfrak{h}_{s}$.

(2) Assume that $s(\lambda)$ is holomorphic at $\bar{\alpha} \in \mathfrak{h}_{s}$. Consider a new operator function

$$
s^{(\alpha)}(\zeta)=s\left(\frac{\zeta+\bar{\alpha}}{1+\alpha \zeta}\right)
$$

Since $s^{(\alpha)}(\cdot) \in \mathcal{S}_{K}\left(\mathfrak{L}_{1}, \mathfrak{L}_{2}\right)$ and also is holomorphic at 0 there exist a Pontryagin space $\mathfrak{H}$, a simple isometric operator $V^{(\alpha)}$ in $\mathfrak{H}$, and a unitary boundary pair $\left(\mathfrak{L}, \Gamma^{(\alpha)}\right)$ such that the corresponding Weyl function $\Theta^{(\alpha)}(\zeta)$ coincides with $s^{(\alpha)}(\zeta)$.

By Lemma 3.14 the pair $(\mathfrak{L}, \Gamma)$ with $\Gamma:=\Gamma^{(\alpha)} \circ M^{(\alpha)}$ is a unitary boundary pair for the simple isometric operator

$$
V=\operatorname{ker} \Gamma=M^{(-\alpha)} V^{(\alpha)}=\left\{\left(\begin{array}{c}
f+\alpha f^{\prime} \\
\bar{\alpha} f+f^{\prime}
\end{array}\right):\left\{f, f^{\prime}\right\} \in V^{(\alpha)}\right\} .
$$

The domains $V_{*}:=\operatorname{dom} \Gamma$ and $V_{*}^{(\alpha)}:=\operatorname{dom} \Gamma^{(\alpha)}$ of $\Gamma$ and $\Gamma^{(\alpha)}$ are connected by

$$
V_{*}:=\operatorname{dom} \Gamma=M^{(-\alpha)} V_{*}^{(\alpha)}=\left\{\left(\begin{array}{c}
f+\alpha f^{\prime} \\
\bar{\alpha} f+f^{\prime}
\end{array}\right):\left\{f, f^{\prime}\right\} \in V_{*}^{(\alpha)}\right\},
$$

The defect subspace $\mathfrak{N}_{\lambda}\left(V_{*}\right):=\operatorname{ker}\left(V_{*}-\lambda I\right)$ and $\mathfrak{N}_{\omega}\left(V_{*}^{(\alpha)}\right):=\operatorname{ker}\left(V_{*}^{(\alpha)}-\omega I\right)$ are connected by (3.45) and the equivalence (3.46) holds. It follows from (3.46) and Definition 3.9 that the Weyl functions of the unitary boundary pairs $(\mathfrak{L}, \Gamma)$ and $\left(\mathfrak{L}, \Gamma^{(\alpha)}\right)$ are connected by

$$
\Theta(\lambda)=\Theta^{(\alpha)}\left(\frac{\lambda-\bar{\alpha}}{1-\alpha \lambda}\right), \quad \lambda \in \mathfrak{h}_{s}
$$

Setting $\zeta=\frac{\lambda-\bar{\alpha}}{1-\alpha \lambda}$ one obtains $\lambda=\frac{\zeta+\bar{\alpha}}{1+\alpha \zeta}$ and hence by (3.66) and (3.65)

$$
\Theta(\lambda)=\Theta^{(\alpha)}(\zeta)=s^{(\alpha)}(\zeta)=s\left(\frac{\zeta+\bar{\alpha}}{1+\alpha \zeta}\right)=s(\lambda), \quad \lambda \in \mathfrak{h}_{s}
$$

This completes the proof.

Remark 3.18 Notice that a simple isometric operator $V$ in Theorem 3.17 and a unitary boundary pair $\left(\mathfrak{L}_{1} \times \mathfrak{L}_{2}, \Gamma\right)$ are determined by the Weyl function $s(\cdot)$ uniquely, up to a 
unitary equivalence. The latter means that if there is another simple isometric operator $V^{\prime}$ in a Pontryagin space $\mathfrak{H}^{\prime}$ and a unitary boundary pair $\left(\mathfrak{L}_{1} \times \mathfrak{L}_{2}, \Gamma^{\prime}\right)$, such that its Weyl function coincides with $s(\cdot)$, then there exists a unitary operator $W$ from $\mathfrak{H}^{\prime}$ to $\mathfrak{H}^{\prime}$, such that

$$
V^{\prime}=W V W^{-1}, \quad \Gamma^{\prime}=\Gamma W^{-1}
$$

Next Theorem 3.15 is specialized to the case where $\mathfrak{H}$ is a Hilbert space and $(\mathfrak{L}, \Gamma)$ is a unitary boundary pair for an isometric operator $V$ in $\mathfrak{H}$. When combined with Theorem 3.17 we get a general realization result for operator valued Schur functions $s(\cdot) \in \mathcal{S}\left(\mathfrak{L}_{1}, \mathfrak{L}_{2}\right)$ as a Weyl function of a unitary boundary pairs for an isometric operator in a Hilbert space.

Theorem 3.19 Let $\mathfrak{H}$ and $\mathfrak{L}=\mathfrak{L}_{1} \times \mathfrak{L}_{2}$ be Hilbert spaces, let $V$ be an isometric operator in $\mathfrak{H}$, let $(\mathfrak{L}, \Gamma)$ be a unitary boundary pair for $V$ and let $V_{1}, V_{2}$ be defined by (3.5). Then:

(i) $V_{1}^{-1}$ and $V_{2}$ are contractive operators in $\mathbf{B}(\mathfrak{H})$ and they are connected by

$$
V_{2}=V_{1}^{-*}
$$

(ii) The $\gamma$-field $\gamma_{1}(\lambda)$ is holomorphic on $\mathbb{D}$ with values in $\mathbf{B}\left(\mathfrak{L}_{1}, \mathfrak{H}\right)$ and satisfies the identity (3.52) for all $\lambda, \mu \in \mathbb{D}$.

(iii) The $\gamma$-field $\gamma_{2}(\lambda)$ is holomorphic on $\mathbb{D}_{e}$ with values in $\mathbf{B}\left(\mathfrak{L}_{2}, \mathfrak{H}\right)$ and satisfies the identity (3.53) for all $\lambda, \mu \in \mathbb{D}_{e}$.

(iv) The Weyl function $\Theta(\lambda)$ of $V$ corresponding to the boundary pair $(\mathfrak{L}, \Gamma)$ belongs to the Schur class $\mathcal{S}\left(\mathfrak{L}_{1}, \mathfrak{L}_{2}\right)$.

Conversely, for every function $s(\cdot)$ from the Schur class $\mathcal{S}\left(\mathfrak{L}_{1}, \mathfrak{L}_{2}\right)$ there exists a simple isometric operator $V$ in a Hilbert space $\mathfrak{H}$ and a unitary boundary pair $(\mathfrak{L}, \Gamma)$ for $V$ such that the corresponding Weyl function $\Theta(\cdot)$ coincides with $s(\cdot)$.

Proof (i) According to Corollary $3.5 V_{1}^{-1}$ and $V_{2}$ are contractive operators. From item (i) in Theorem 3.15 one concludes that

$$
\mathbb{D} \subseteq \rho\left(V_{2}^{-1}\right) \text { and } \mathbb{D}_{e} \subseteq \rho\left(V_{1}^{-1}\right)
$$

In particular, $0 \in \rho\left(V_{2}^{-1}\right)$ so that $V_{2} \in \mathbf{B}(\mathfrak{H})$. On the other hand, by Proposition 3.4 one has the inclusion $V_{2} \subseteq V_{1}^{-*}$. Since $\mathbb{D}_{e} \subseteq \rho\left(V_{1}^{-*}\right)$, the equality (3.67) must prevail and, hence, $V_{1}^{-*} \in \mathbf{B}(\mathfrak{H}) \Leftrightarrow V_{1}^{-1} \in \mathbf{B}(\mathfrak{H})$.

The assertions (ii)-(iv) are now obtained directly from Theorem 3.15.

The last statement follows from Theorem 3.17 by taking $\kappa=0$.

\subsection{Classification of Unitary Boundary Pairs}

We start by collecting some main properties of unitary boundary pairs in the next proposition. 
Proposition 3.20 Let $V$ be an isometric operator in the Pontryagin space $\mathfrak{H}$ and let $(\mathfrak{L}, \Gamma)$ be a unitary boundary pair for $V$. Then $\Gamma$ and its components $\Gamma_{1}$ and $\Gamma_{2}$ defined by (3.3), (3.4) admit the following properties:

(i) $\Gamma_{1}$ and $\Gamma_{2}$ are closed linear relations with $\operatorname{ran} \Gamma_{1}=\mathfrak{L}_{1}, \operatorname{ran} \Gamma_{2}=\mathfrak{L}_{2}$ and, moreover, mul $\Gamma_{1}=\{0\} \Leftrightarrow \operatorname{mul} \Gamma_{2}=\{0\} \Leftrightarrow \operatorname{mul} \Gamma=\{0\}$;

(ii) $V_{1}=\operatorname{ker} \Gamma_{1}$ and $V_{2}=\operatorname{ker} \Gamma_{2}$ have nonempty resolvent sets and $V_{1}=V_{2}^{-[*]}$;

(iii) $V_{*}=\operatorname{dom} \Gamma$ admits the decompositions

$$
\begin{array}{ll}
V_{*}=V_{1} \widehat{+} \widehat{\mathfrak{N}}_{\lambda}\left(V_{*}\right), & \lambda \in \mathcal{D}=\rho\left(V_{1}\right) \cap \mathbb{D}, \\
V_{*}=V_{2} \widehat{+} \widehat{\mathfrak{N}}_{\lambda}\left(V_{*}\right), & \lambda \in \mathcal{D}_{e}=\rho\left(V_{2}\right) \cap \mathbb{D}_{e} .
\end{array}
$$

Proof (i) These properties were proven in Proposition 3.4.

(ii) The fact that $\rho\left(V_{1}\right)$ and $\rho\left(V_{2}\right)$ are nonempty and the equality $V_{2}=V_{1}^{-*}$ were proven in Theorem 3.15.

(iii) This is a direct consequence of (ii); see e.g. [29, Lemma 4.1].

Remark 3.21 Proposition 3.20 shows that in a Pontryagin space $\mathfrak{H}$ every unitary boundary pair $(\mathfrak{L}, \Gamma$ ) (as well as its transposed boundary pair) of an isometric operator $V$ can be seen as an analog of so-called ( $B$-)generalized boundary triple, since the component mappings $\Gamma_{1}$ and $\Gamma_{2}$ are surjective and the corresponding kernels $V_{1}=\operatorname{ker} \Gamma_{1}$ and $V_{2}=\operatorname{ker} \Gamma_{2}$ are closed extensions of $V$ with nonempty resolvent sets; see $[19,26]$ and [22-24] for some further developments.

Proposition 3.22 The following relations hold:

$$
\begin{aligned}
& S_{\mu}^{\Theta}(\lambda):=\frac{I-\Theta(\mu)^{*} \Theta(\lambda)}{1-\lambda \bar{\mu}}=\gamma_{1}(\mu)^{[*]} \gamma_{1}(\lambda), \quad \lambda, \mu \in \mathcal{D} ; \\
& S_{\mu}^{\Theta}(\lambda):=\frac{I-\Theta^{\#}(\mu)^{*} \Theta^{\#}(\lambda)}{1-\lambda \bar{\mu}}=-\gamma_{2}(\mu)^{[*]} \gamma_{2}(\lambda), \quad \lambda, \mu \in \mathcal{D}_{e} ; \\
& S_{\mu}^{\Theta}(\lambda):=\frac{\Theta(\lambda)-\Theta^{\#}(\mu)^{*}}{1-\lambda \bar{\mu}}=-\gamma_{2}(\mu)^{[*]} \gamma_{1}(\lambda), \quad \lambda \in \mathcal{D}, \mu \in \mathcal{D}_{e} ; \\
& S_{\mu}^{\Theta}(\lambda):=\frac{\Theta^{\#}(\lambda)-\Theta(\mu)^{*}}{1-\lambda \bar{\mu}}=\gamma_{1}(\mu)^{[*]} \gamma_{2}(\lambda), \quad \lambda \in \mathcal{D}_{e}, \mu \in \mathcal{D} .
\end{aligned}
$$

Proof Let $\lambda, \mu \in \mathcal{D}$ and $u_{1}, v_{1} \in \mathfrak{L}_{1}$. Then by (3.50)

$$
\left\{\left(\begin{array}{c}
\gamma_{1}(\lambda) u_{1} \\
\lambda \gamma_{1}(\lambda) u_{1}
\end{array}\right),\left(\begin{array}{c}
u_{1} \\
\Theta(\lambda) u_{1}
\end{array}\right)\right\} \in \Gamma, \quad\left\{\left(\begin{array}{c}
\gamma_{1}(\mu) v_{1} \\
\mu \gamma_{1}(\mu) v_{1}
\end{array}\right),\left(\begin{array}{c}
v_{1} \\
\Theta(\mu) v_{1}
\end{array}\right)\right\} \in \Gamma
$$

and the identity (3.9) applied to these vectors yields

$$
(1-\lambda \bar{\mu})\left[\gamma_{1}(\lambda) u_{1}, \gamma_{1}(\mu) v_{1}\right]_{\mathfrak{H}}=\left[u_{1}, v_{1}\right]_{\mathfrak{L}_{1}}-\left[\Theta(\lambda) u_{1}, \Theta(\mu) v_{1}\right]_{\mathfrak{L}_{2}}
$$

This proves the equality (3.68). 
Let now $\lambda \in \mathcal{D}, \mu \in \mathcal{D}_{e}$ and let $u_{1} \in \mathfrak{L}_{1}$ and $v_{2} \in \mathfrak{L}_{2}$. Then by (3.50)

$$
\left\{\left(\begin{array}{c}
\gamma_{1}(\lambda) u_{1} \\
\lambda \gamma_{1}(\lambda) u_{1}
\end{array}\right),\left(\begin{array}{c}
u_{1} \\
\Theta(\lambda) u_{1}
\end{array}\right)\right\} \in \Gamma, \quad\left\{\left(\begin{array}{c}
\gamma_{2}(\mu) v_{2} \\
\mu \gamma_{2}(\mu) v_{2}
\end{array}\right),\left(\begin{array}{c}
\Theta^{\#}(\mu) v_{2} \\
v_{2}
\end{array}\right)\right\} \in \Gamma .
$$

By applying (3.9) to these vectors one arrives at

$$
(1-\lambda \bar{\mu})\left[\gamma_{1}(\lambda) u_{1}, \gamma_{2}(\mu) v_{2}\right]_{\mathfrak{H}}=\left[u_{1}, \Theta^{\#}(\mu) v_{2}\right]_{\mathfrak{L}_{1}}-\left[\Theta(\lambda) u_{1}, v_{2}\right]_{\mathfrak{L}_{2}} .
$$

This yields (3.70). The proof of (3.69) and (3.71) is analogous.

Proposition 3.23 Let $(\mathfrak{L}, \Gamma)$ be a unitary boundary pair for an isometric operator $V$ in the Pontryagin space $\mathfrak{H}$. Then

$$
\operatorname{mul} \Gamma_{1}=\operatorname{ker} \gamma_{1}(\lambda)(\lambda \in \mathcal{D}) \text { and } \operatorname{mul} \Gamma_{2}=\operatorname{ker} \gamma_{2}(\lambda)\left(\lambda \in \mathcal{D}_{e}\right) \text {. }
$$

Moreover, the following statements are equivalent:

(i) $\Gamma$ is single valued (i.e. mul $\Gamma=\{0\}$ );

(ii) $\operatorname{ran} \Gamma$ is dense in $\mathfrak{L}_{1} \times \mathfrak{L}_{2}$;

(iii) $\operatorname{ker} \gamma_{1}(\lambda)=\{0\}$ for some (equivalently for all) $\lambda \in \mathcal{D}$;

(iv) $\operatorname{ker} \gamma_{2}(\lambda)=\{0\}$ for some (equivalently for all) $\lambda \in \mathcal{D}_{e}$.

If, in addition, the operator $V$ is simple then the conditions (i)-(iv) are equivalent to

(v) $\bigcap_{\mu \in \mathcal{D} \cup \mathcal{D}_{e}} \operatorname{ker} S_{\mu}^{\Theta}(\lambda)=\{0\}$ for some (equivalently for all) $\lambda \in \mathcal{D} \cup \mathcal{D}_{e}$.

Proof (i) $\Leftrightarrow$ (ii) This is item (ii) in Proposition 3.2.

(i) $\Leftrightarrow$ (iii), (iv) The formula (3.11) and Definition 3.8 of the $\gamma$-fields shows that ker $\gamma_{1}(\lambda)=\operatorname{mul} \Gamma_{1}$ for $\lambda \in \mathcal{D}$ and $\operatorname{ker} \gamma_{2}(\lambda)=\operatorname{mul} \Gamma_{2}$ for $\lambda \in \mathcal{D}_{e}$. Now the statement follows from item (i) in Proposition 3.20.

To prove the statement in (v) first observe that with $\lambda \in \mathcal{D}$ the inclusions

$$
\operatorname{ker} \gamma_{1}(\lambda) \subseteq \operatorname{ker} S_{\mu}^{\Theta}(\lambda) \text { for all } \mu \in \mathcal{D} \cup \mathcal{D}_{e}
$$

are clear from (3.68) and (3.70) in Proposition 3.22. Thus

$$
\operatorname{ker} \gamma_{1}(\lambda) \subseteq \bigcap_{\mu \in \mathcal{D} \cup \mathcal{D}_{e}} \operatorname{ker} S_{\mu}^{\Theta}(\lambda), \quad \lambda \in \mathcal{D}
$$

As to the reverse inclusion apply Proposition 3.22 again to see that for all $\lambda, \mu \in \mathcal{D}$ and $u_{1}, v_{1} \in \mathfrak{L}_{1}$,

$$
\left(\mathrm{S}_{\mu}^{\Theta}(\lambda) u_{1}, v_{1}\right)_{\mathfrak{L}_{1}}=\left[\gamma_{1}(\lambda) u_{1}, \gamma_{1}(\mu) v_{1}\right]_{\mathfrak{H}}
$$

and for all $\lambda \in \mathcal{D}, v \in \mathcal{D}_{e}$ and $u_{1} \in \mathfrak{L}_{1}, v_{2} \in \mathfrak{L}_{2}$,

$$
\left(\mathrm{S}_{\mu}^{\Theta}(\lambda) u_{1}, v_{2}\right)_{\mathfrak{L}_{2}}=\left[\gamma_{1}(\lambda) u_{1}, \gamma_{2}(\mu) v_{2}\right]_{\mathfrak{H}}
$$


Now assume that the operator $V$ is simple. Then

$$
\overline{\operatorname{span}}\left\{\gamma_{1}(\mu) \mathfrak{L}_{1}, \gamma_{2}(\nu) \mathfrak{L}_{2}: \mu \in \mathcal{D}, \nu \in \mathcal{D}_{e}\right\}=\mathfrak{H}
$$

and by virtue of (3.73) and (3.74) the reverse inclusion in (3.72) follows. This proves the equivalence (iv) $\Leftrightarrow$ (v) for $\lambda \in \mathcal{D}$ when $V$ is simple. Similarly one proves the equivalence (iii) $\Leftrightarrow(\mathrm{v})$ for $\lambda \in \mathcal{D}_{e}$.

Proposition 3.24 Let $(\mathfrak{L}, \Gamma)$ be a unitary boundary pair for an isometric operator $V$ in the Pontryagin space $\mathfrak{H}$. Then the following statements are equivalent:

(i) $\operatorname{ran} \Gamma=\mathfrak{L}_{1} \times \mathfrak{L}_{2}$;

(ii) $\operatorname{dom} \Gamma=V^{-[*]}$ and $\operatorname{mul} \Gamma=\{0\}$;

(iii) $\operatorname{ker} \gamma_{1}(\lambda)=\{0\}$ and $\operatorname{ran} \gamma_{1}(\lambda)$ is closed for some (equivalently for all) $\lambda \in \mathcal{D}$;

(iv) $\operatorname{ker} \gamma_{2}(\lambda)=\{0\}$ and $\operatorname{ran} \gamma_{2}(\lambda)$ is closed for some (equivalently for all) $\lambda \in \mathcal{D}_{e}$. If $0 \in \rho\left(S_{\lambda}^{\Theta}(\lambda)\right)$ for some $\lambda \in \mathcal{D} \cup \mathcal{D}_{e}$, then the conditions ( $\left.i\right)$ - (iv) hold.

If one of the condition $(i)-(i v)$ is satisfied then $(\mathfrak{L}, \Gamma)$ is an ordinary boundary pair for $V$.

Proof (i) $\Leftrightarrow$ (ii) This is item (iii) in Proposition 3.2.

(ii) $\Leftrightarrow$ (iii), (iv) Definition 3.8 of the $\gamma$-fields shows that $\operatorname{ran} \gamma_{1}(\lambda)=\mathfrak{N}_{\lambda}\left(V_{*}\right)$ for $\lambda \in \mathcal{D}$ and $\operatorname{ran} \gamma_{2}(\lambda)=\mathfrak{N}_{\lambda}\left(V_{*}\right)$ for $\lambda \in \mathcal{D}_{e}$. Now the decompositions of $V_{*}=\operatorname{dom} \Gamma$ in item (iii) of Proposition 3.20 imply that $\mathfrak{N}_{\lambda}\left(V_{*}\right)$ is closed for some $\lambda \in \mathcal{D} \cup \mathcal{D}_{e}$ if and only if $V_{*}=V^{-[*]}$. This combined with Proposition 3.23 gives the stated equivalences.

The last implication follows from Proposition 3.22. Indeed, if $0 \in \rho\left(\mathrm{S}_{\lambda}^{\Theta}(\lambda)\right)$ for some $\lambda \in \mathcal{D}$, then by Proposition $3.23 \operatorname{ker} \gamma_{1}(\lambda)=\{0\}$ and by (3.68) $\operatorname{ran} \gamma_{1}(\lambda)$ is closed. Similarly, if $0 \in \rho\left(\mathrm{S}_{\lambda}^{\Theta}(\lambda)\right)$ for some $\lambda \in \mathcal{D}_{e}$, then again by Proposition 3.23 ker $\gamma_{2}(\lambda)=\{0\}$ and by (3.69) $\operatorname{ran} \gamma_{2}(\lambda)$ is closed.

Finally, the fact that $(\mathfrak{L}, \Gamma)$ is an ordinary boundary pair for $V$ is clear from the properties in (ii).

The next example shows that the condition (v) in Proposition 3.23 cannot be replaced by a single condition $\operatorname{ker} S_{\lambda}^{\Theta}(\lambda)=\{0\}$.

Example 3.25 Let $\mathfrak{H}=\mathbb{C}^{4}$ with the skew-diagonal fundamental symmetry $J=$ $\left(\delta_{j, 5-k}\right)_{j, k=1}^{4}$, where $\delta_{j, k}$ is the Kronecker delta and let us set $e_{j}=\left(\delta_{j, k}\right)_{k=1}^{4}$, $(j=1,2,3,4)$. Let $V$ be an isometry in $\mathfrak{H}$ which maps $e_{1}$ into $e_{2}$. Then the defect subspaces of $V$

$$
\mathfrak{N}_{\lambda}=\left\{\left(f_{1}, f_{2}, f_{3}, \lambda f_{3}\right)^{\top}: f_{1}, f_{2}, f_{3} \in \mathbb{C}\right\}
$$

are degenerate for all $\lambda \in \mathbb{C}$ and thus, the operator $V$ is not standard.

The linear relation $V^{-[*]}$ consists of vectors

$$
\widehat{f}=\left(\begin{array}{c}
f \\
f^{\prime}
\end{array}\right), \quad \text { where } \quad f=\sum_{j=1}^{4} f_{j} e_{j}, \quad f^{\prime}=\sum_{j=1}^{4} f_{j}^{\prime} e_{j}, \quad f_{3}^{\prime}=f_{4} .
$$


Therefore, the left part of the identity (3.2) for $\widehat{f}=\widehat{g}$ takes the form

$$
\left(f_{1}-f_{2}^{\prime}\right) \overline{f_{4}}+f_{2} \overline{f_{3}}+f_{3} \overline{f_{2}}+f_{4}\left(\overline{f_{1}}-\overline{f_{2}^{\prime}}\right)-f_{1}^{\prime} \overline{f_{4}^{\prime}}-f_{4}^{\prime} \overline{f_{1}^{\prime}}
$$

and can be rewritten in the diagonal form

$$
\begin{aligned}
& \frac{1}{2}\left\{\left|f_{1}^{\prime}-f_{4}^{\prime}\right|^{2}-\left|f_{1}^{\prime}+f_{4}^{\prime}\right|^{2}+\left|f_{1}-f_{2}^{\prime}+f_{4}\right|^{2}-\left|f_{1}-f_{2}^{\prime}-f_{4}\right|^{2}\right. \\
& \left.\quad+\left|f_{2}+f_{3}\right|^{2}-\left|f_{2}-f_{3}\right|^{2}\right\} .
\end{aligned}
$$

Hence a single-valued boundary triple $\left(\mathfrak{L}_{1} \oplus \mathfrak{L}_{2}, \Gamma_{1}, \Gamma_{2}\right)$ can be chosen as follows

$$
\mathfrak{L}_{1}=\mathfrak{L}_{2}=\mathbb{C}^{3}, \quad \Gamma_{1} \widehat{f}=\frac{1}{\sqrt{2}}\left(\begin{array}{c}
f_{1}^{\prime}-f_{4}^{\prime} \\
f_{1}-f_{2}^{\prime}+f_{4} \\
f_{2}+f_{3}
\end{array}\right), \quad \Gamma_{2} \widehat{f}=\frac{1}{\sqrt{2}}\left(\begin{array}{c}
f_{1}^{\prime}+f_{4}^{\prime} \\
f_{1}-f_{2}^{\prime}-f_{4} \\
f_{2}-f_{3}
\end{array}\right) .
$$

Then for $|\lambda|<1$ one obtains from (3.75) and (3.76)

$$
\Theta(\lambda)=\frac{1}{3 \lambda^{2}}\left(\begin{array}{ccc}
\lambda^{2} & 2 \lambda^{3} & 2 \lambda^{4} \\
2 \lambda & \lambda^{2} & -2 \lambda^{3} \\
2 & -2 \lambda & \lambda^{2}
\end{array}\right)
$$

and hence

$$
\mathrm{S}_{\omega}^{\Theta}(\lambda)=\frac{-2}{9 \lambda^{2} \bar{\omega}^{2}} \Omega^{*}\left(\begin{array}{ccc}
2(1+2 \lambda \bar{\omega}) & -(2+\lambda \bar{\omega}) & 1-\lambda \bar{\omega} \\
-(2+\lambda \bar{\omega}) & 2(1-\lambda \bar{\omega}) & -(1+2 \lambda \bar{\omega}) \\
1-\lambda \bar{\omega} & -(1+2 \lambda \bar{\omega}) & -2(2+\lambda \bar{\omega})
\end{array}\right) \Lambda
$$

where $\Lambda=\operatorname{diag}\left(1, \lambda, \lambda^{2}\right), \Omega=\operatorname{diag}\left(1, \omega, \omega^{2}\right)$. Notice that in this example $\operatorname{det} S_{\omega}^{\Theta}(\lambda) \equiv 0$ for all $\lambda, \omega \in \mathbb{D} \backslash\{0\}$, while $\bigcap_{\omega \in \mathbb{D} \backslash\{0\}} \operatorname{ker} S_{\omega}^{\Theta}(\lambda)=\{0\}$. In fact, for every pair $\omega_{1}, \omega_{2} \in \mathbb{D} \backslash\{0\}, \omega_{1} \neq \omega_{2}$, one gets $\operatorname{ker} S_{\omega_{1}}^{\Theta}(\lambda) \cap \operatorname{ker} S_{\omega_{2}}^{\Theta}(\lambda)=\{0\}$.

Remark 3.26 Let $A$ be a closed symmetric operator in a Pontryagin space $\mathfrak{H}$ with equal defect numbers, and let $\pm i \notin \sigma_{p}(A)$. Then its Cayley transform $V=(A-i I)(A+$ $i I)^{-1}$ is an isometric operator in $\mathfrak{H}$.

Let $\left(\mathfrak{L}_{1} \oplus \mathfrak{L}_{2}, \Gamma\right)$ be a boundary pair for $V$ with $V_{*}=\operatorname{dom} \Gamma$ such that $\mathfrak{L}_{1}=\mathfrak{L}_{2}=$ : $\mathcal{H}$. Define the Krel̆n spaces $\left(\mathfrak{H}^{2}, \mathbb{[} \cdot, \cdot \mathbb{1}_{\mathfrak{H}^{2}}\right)$ and $\left(\mathcal{H}^{2}, \llbracket\left[\cdot, \cdot \rrbracket_{\mathcal{H}^{2}}\right)\right.$ with the inner products

$$
\begin{gathered}
\llbracket \widehat{f}, \widehat{f} \rrbracket_{\mathfrak{H}^{2}}=-i\left(\left[f^{\prime}, f\right]_{\mathfrak{H}}-\left[f, f^{\prime}\right]_{\mathfrak{H}}\right), \quad \widehat{f}=\left(\begin{array}{c}
f \\
f^{\prime}
\end{array}\right) \in \mathfrak{H}^{2}, \\
\llbracket \widehat{u}, \widehat{u} \rrbracket_{\mathcal{H}^{2}}=-i\left(\left(u^{\prime}, u\right)_{\mathcal{H}}-\left(u, u^{\prime}\right)_{\mathcal{H}}\right), \quad \widehat{u}=\left(\begin{array}{c}
u \\
u^{\prime}
\end{array}\right) \in \mathcal{H}^{2} .
\end{gathered}
$$


Then the Cayley transform determines the unitary operator from the KreĬn space $\left(\mathfrak{H}^{2}, \llbracket\left[\cdot, \cdot \rrbracket_{\mathfrak{H}^{2}}\right)\right.$ to the Kreı̆n space $\left(\mathfrak{H}^{2}, J_{\mathfrak{H}^{2}}\right)$ defined in Sect. 3.1,

$$
C=\frac{1}{\sqrt{2}}\left(\begin{array}{cc}
i & 1 \\
-i & 1
\end{array}\right):\left(\mathfrak{H}^{2}, \mathbb{[} \cdot, \cdot \mathbb{1}_{\mathfrak{H}^{2}}\right) \rightarrow\left(\mathfrak{H}^{2}, J_{\mathfrak{H}^{2}}\right)
$$

and similarly with $\mathfrak{L}=\mathfrak{L}_{1} \times \mathfrak{L}_{2}$ it determines a unitary mapping from the Kreı̆n space $\left(\mathcal{H}^{2}, \llbracket\left[\cdot, \rrbracket_{\mathcal{H}^{2}}\right)\right.$ to the Kreĭn space $\left(\mathfrak{L}, J_{\mathfrak{L}}\right)$,

$$
C=\frac{1}{\sqrt{2}}\left(\begin{array}{cc}
i & 1 \\
-i & 1
\end{array}\right):\left(\mathcal{H}^{2}, \llbracket \cdot \cdot \cdot \mathbb{H}_{\mathcal{H}^{2}}\right) \rightarrow\left(\mathfrak{L}, J_{\mathfrak{L}}\right)
$$

It follows that the linear relation

$$
\widetilde{\Gamma}=C^{-1} \circ \Gamma \circ C=\left\{\{\widehat{f}, \widehat{u}\}:=\left\{\left(\begin{array}{c}
i g^{\prime}-i g \\
g^{\prime}+g
\end{array}\right),\left(\begin{array}{c}
i v^{\prime}-i v \\
v^{\prime}+v
\end{array}\right)\right\}:\left\{\left(\begin{array}{c}
g \\
g^{\prime}
\end{array}\right),\left(\begin{array}{c}
v \\
v^{\prime}
\end{array}\right)\right\} \in \Gamma\right\}
$$

is unitary from $\left(\mathfrak{H}^{2}, \llbracket \cdot \cdot \cdot \mathbb{H}_{\mathfrak{H}^{2}}\right)$ to $\left(\mathcal{H}^{2}, \llbracket \cdot \cdot, \cdot \rrbracket_{\mathcal{H}^{2}}\right)$ with the kernel ker $\widetilde{\Gamma}=A$ and the domain

$$
\operatorname{dom} \widetilde{\Gamma}=C^{-1} V_{*}=\left\{\widehat{f}=\left(\begin{array}{c}
i g^{\prime}-i g \\
g^{\prime}+g
\end{array}\right):\left(\begin{array}{c}
g \\
g^{\prime}
\end{array}\right) \in V_{*}\right\}
$$

Since the mapping $\widetilde{\Gamma}$ is isometric from $\left(\mathfrak{H}^{2}, \llbracket\left[\cdot, \cdot \mathbb{H}_{\mathfrak{H}^{2}}\right)\right.$ to $\left(\mathcal{H}^{2}, \llbracket \cdot, \cdot \mathbb{H}_{\mathcal{H}^{2}}\right)$ the following (Green's) identity

$$
\left[f^{\prime}, f\right]_{\mathfrak{H}}-\left[f, f^{\prime}\right]_{\mathfrak{H}}=\left[u^{\prime}, u\right]_{\mathcal{H}}-\left[u, u^{\prime}\right]_{\mathcal{H}}
$$

holds for all $\{\widehat{f}, \widehat{u}\} \in \widetilde{\Gamma}$ and due to $[19,22]$ the unitarity of $\widetilde{\Gamma}$ means that $\left(\mathcal{H}^{2}, \widetilde{\Gamma}\right)$ is a unitary boundary pair for the symmetric operator $A$. This boundary pair becomes ordinary when the mapping $\widetilde{\Gamma}$ is surjective or, equivalently, when $\left(\mathcal{H}^{2}, \Gamma\right)$ is an ordinary boundary pair for $V$.

Finally the main results in this subsection are specialized to unitary boundary pairs of Hilbert space isometries. In a Hilbert space setting the properties of the $\gamma$-fields can be connected more directly to the properties of the Weyl function.

Proposition 3.27 Let $(\mathfrak{L}, \Gamma)$ be a unitary boundary pair for an isometric operator $V$ in the Pontryagin space $\mathfrak{H}$. Then

$$
\operatorname{mul} \Gamma_{1}=\operatorname{ker} \gamma_{1}(\lambda), \lambda \in \mathbb{D}, \quad \text { and } \operatorname{mul} \Gamma_{2}=\operatorname{ker} \gamma_{2}(\lambda), \lambda \in \mathbb{D}_{e} \text {, }
$$

and $(\mathfrak{L}, \Gamma)$ admits the following further properties.

(a) The following statements are equivalent:

(i) $\Gamma$ is single valued (i.e. mul $\Gamma=\{0\}$ );

(ii) ran $\Gamma$ is dense in $\mathfrak{L}_{1} \times \mathfrak{L}_{2}$; 
(iii) $\operatorname{ker} \gamma_{1}(\lambda)=\{0\}$ for some (equivalently for all) $\lambda \in \mathbb{D}$;

(iv) $\operatorname{ker} \gamma_{2}(\lambda)=\{0\}$ for some (equivalently for all) $\lambda \in \mathbb{D}_{e}$;

(v) $\operatorname{ker} S_{\lambda}^{\Theta}(\lambda)=\{0\}$ for some (equivalently for all) $\lambda \in \mathbb{D} \cup \mathbb{D}_{e}$.

(b) Moreover, the following statements are equivalent:

(i) $(\mathfrak{L}, \Gamma)$ reduces to an ordinary boundary triple $\left(\mathfrak{L}, \Gamma_{1}, \Gamma_{2}\right)$ for $V$;

(ii) $\operatorname{dom} \Gamma=V^{-[*]}$ and $\operatorname{mul} \Gamma=\{0\}$;

(iii) $\operatorname{ran} \Gamma=\mathfrak{L}_{1} \times \mathfrak{L}_{2}$;

(iv) $\operatorname{ker} \gamma_{1}(\lambda)=\{0\}$ and $\operatorname{ran} \gamma_{1}(\lambda)$ is closed for some (equivalently for all) $\lambda \in \mathbb{D}$;

(v) ker $\gamma_{2}(\lambda)=\{0\}$ and $\operatorname{ran} \gamma_{2}(\lambda)$ is closed for some (equivalently for all) $\lambda \in$ $\mathbb{D}_{e}$;

(vi) $0 \in \rho\left(S_{\lambda}^{\Theta}(\lambda)\right.$ ), i.e., $\|\Theta(\lambda)\|<1$ for some (equivalently for all) $\lambda \in \mathbb{D} \cup \mathbb{D}_{e}$.

Proof (a) The equivalences (i)-(iv) follow from Proposition 3.23; see also Theorem 3.19. To see the equivalence with item (v) apply Proposition 3.22 with $\lambda=\mu$ :

$$
\begin{gathered}
S_{\lambda}^{\Theta}(\lambda)=\frac{I-\Theta(\lambda)^{*} \Theta(\lambda)}{1-|\lambda|^{2}}=\gamma_{1}(\lambda)^{*} \gamma_{1}(\lambda) \geq 0, \quad \lambda \in \mathbb{D} ; \\
S_{\lambda}^{\Theta}(\lambda)=\frac{I-\Theta^{\#}(\lambda)^{*} \Theta^{\#}(\lambda)}{1-|\lambda|^{2}}=-\gamma_{2}(\lambda)^{*} \gamma_{2}(\lambda) \leq 0, \quad \lambda \in \mathbb{D}_{e} .
\end{gathered}
$$

In the present Hilbert space case these identities lead to

$$
\operatorname{ker} S_{\lambda}^{\Theta}(\lambda)=\operatorname{ker} \gamma_{1}(\lambda), \lambda \in \mathbb{D} ; \quad \operatorname{ker} S_{\lambda}^{\Theta}(\lambda)=\operatorname{ker} \gamma_{2}(\lambda), \lambda \in \mathbb{D}_{e}
$$

This implies the equivalence of (iii), (iv) and (v) in part (a).

(b) Here the equivalence of (i) and (ii) holds just by the definition of an ordinary boundary triple (see [41]). The equivalences (ii)-(v) are obtained from Proposition 3.24 (cf. also Theorem 3.19). To see the equivalence with item (vi) apply the identities (3.77), (3.78):

$$
\begin{array}{lll}
0 \in \rho\left(I-\Theta(\lambda)^{*} \Theta(\lambda)\right) & \Longleftrightarrow \quad 0 \in \rho\left(\gamma_{1}(\lambda)^{*} \gamma_{1}(\lambda)\right), \quad \lambda \in \mathbb{D} ; \\
0 \in \rho\left(I-\Theta^{\#}(\lambda)^{*} \Theta^{\#}(\lambda)\right) \Longleftrightarrow 0 \in \rho\left(\gamma_{2}(\lambda)^{*} \gamma_{2}(\lambda)\right), & \lambda \in \mathbb{D}_{e} .
\end{array}
$$

Thus $I-\Theta(\lambda)^{*} \Theta(\lambda)$ and $I-\Theta^{\#}(\lambda)^{*} \Theta^{\#}(\lambda)$ are uniformly positive or, equivalently, $\|\Theta(\lambda)\|<1$ and $\left.\| \Theta^{\#}(\lambda)\right) \|<1$. This completes the proof.

Notice that part (b) of Proposition 3.27 contains the properties and generality that can be attained when applying (ordinary) boundary triples for isometric operators which have been introduced and studied in [41,42].

Remark 3.28 An analog of boundary triple in scattering form (3.2) is encountered in [17], where extension theory of multiplication operators in indefinite de Branges spaces was developed. The role of the Weyl function in that work is played by de Branges matrix. 


\section{Extension Theory and Generalized Coresolvents}

An extension $\widetilde{V}$ of the isometric operator $V$ is called proper, if $V \subsetneq \widetilde{V} \subsetneq V^{-[*]}$. The set of proper extensions of $V$ was parametrized in [5] via an ordinary boundary triple. In the present section we consider extensions $\widetilde{V}$ of the isometric operator $V$, which are proper with respect to a given unitary boundary pair $(\mathfrak{L}, \Gamma)$, i.e.

$$
V \subsetneq \widetilde{V} \subsetneq V_{*}=\operatorname{dom} \Gamma
$$

For such extensions we prove sufficient conditions for regularity of a point $\lambda$, find formulas for their coresolvents and then apply them for a description of generalized coresolvents of the isometric operator $V$.

\subsection{A Preparatory Lemma}

Lemma 4.1 Let $V: \mathfrak{H} \rightarrow \mathfrak{H}$ be an isometric operator and let $\Pi=(\mathfrak{L}, \Gamma)$ be a unitary boundary pair for $V$. Then:

(i) For every $\left(\begin{array}{l}f_{1} \\ f_{1}^{\prime}\end{array}\right) \in V_{1}$ and $\lambda \in \mathcal{D}$, as defined in (3.48) one has

$$
\left\{\left(\begin{array}{l}
f_{1} \\
f_{1}^{\prime}
\end{array}\right), \frac{1}{\lambda} \gamma_{2}^{\#}(\lambda)\left(f_{1}^{\prime}-\lambda f_{1}\right)\right\} \in \Gamma_{2}
$$

If $0 \in \mathcal{D}$ then the formula (4.2) for $\lambda=0$ takes the form

$$
\left\{\left(\begin{array}{l}
f_{1} \\
f_{1}^{\prime}
\end{array}\right),\left(\gamma_{2}^{\#}\right)^{\prime}(0) f_{1}\right\} \in \Gamma_{2} .
$$

(ii) For every $\left(\begin{array}{l}f_{2} \\ f_{2}^{\prime}\end{array}\right) \in V_{2}$ and $\lambda \in \mathcal{D}_{e}$ one has

$$
\left\{\left(\begin{array}{c}
f_{2} \\
f_{2}^{\prime}
\end{array}\right),-\frac{1}{\lambda} \gamma_{1}^{\#}(\lambda)\left(f_{1}^{\prime}-\lambda f_{1}\right)\right\} \in \Gamma_{1}
$$

Proof (i) Using Definitions 3.8, 3.9 it is seen that

$$
\left\{\left(\begin{array}{c}
\gamma_{2}\left(\frac{1}{\bar{\lambda}}\right) u_{2} \\
\frac{1}{\bar{\lambda}} \gamma_{2}\left(\frac{1}{\bar{\lambda}}\right) u_{2}
\end{array}\right),\left(\begin{array}{c}
\Theta\left(\frac{1}{\bar{\lambda}}\right)^{*} u_{2} \\
u_{2}
\end{array}\right)\right\}, \quad\left\{\left(\begin{array}{c}
f_{1} \\
f_{1}^{\prime}
\end{array}\right),\left(\begin{array}{c}
0 \\
v_{2}
\end{array}\right)\right\} \in \Gamma,
$$

for all $u_{2} \in \mathfrak{L}_{2}$, and some $v_{2} \in \mathfrak{L}_{2}, \lambda \in \mathcal{D} \backslash\{0\}$. By applying the identity (3.2) to these elements one obtains

$$
\left[\gamma_{2}\left(\frac{1}{\bar{\lambda}}\right) u_{2}, f_{1}\right]_{\mathfrak{H}}-\frac{1}{\bar{\lambda}}\left[\gamma_{2}\left(\frac{1}{\bar{\lambda}}\right) u_{2}, f_{1}^{\prime}\right]_{\mathfrak{H}}=-\left(u_{2}, v_{2}\right) \mathfrak{L}_{2},
$$


or, equivalently,

$$
\left(u_{2}, \gamma_{2}\left(\frac{1}{\bar{\lambda}}\right)^{*}\left(\frac{1}{\lambda} f_{1}^{\prime}-f_{1}\right)\right)_{\mathfrak{L}_{2}}=\left(u_{2}, v_{2}\right)_{\mathfrak{L}_{2}} .
$$

Since $u_{2} \in \mathfrak{L}_{2}$ is arbitrary this implies the equality

$$
v_{2}=\frac{1}{\lambda} \gamma_{2}^{\#}(\lambda)\left(f_{1}^{\prime}-\lambda f_{1}\right)
$$

which in combination with (4.5) yields (4.2).

The equality (4.3) is implied by (4.2) and (3.49).

(ii) Similarly, applying (3.2) to the vectors

$$
\left\{\left(\begin{array}{c}
\gamma_{1}\left(\frac{1}{\bar{\lambda}}\right) u_{1} \\
\frac{1}{\bar{\lambda}} \gamma_{1}\left(\frac{1}{\bar{\lambda}}\right) u_{1}
\end{array}\right),\left(\begin{array}{c}
u_{1} \\
\Theta\left(\frac{1}{\bar{\lambda}}\right) u_{1}
\end{array}\right)\right\},\left\{\left(\begin{array}{c}
f_{2} \\
f_{2}^{\prime}
\end{array}\right),\left(\begin{array}{c}
v_{1} \\
0
\end{array}\right)\right\} \in \Gamma
$$

where $u_{1}, v_{1} \in \mathfrak{L}_{1}, f_{2}, f_{2}^{\prime} \in \mathfrak{H}, \lambda \in \mathcal{D}_{e}$, one obtains

$$
\left[\gamma_{1}\left(\frac{1}{\bar{\lambda}}\right) u_{1}, f_{2}\right]_{\mathfrak{H}}-\frac{1}{\bar{\lambda}}\left[\gamma_{1}\left(\frac{1}{\bar{\lambda}}\right) u_{1}, f_{2}^{\prime}\right]_{\mathfrak{H}}=\left(u_{1}, v_{1}\right)_{\mathfrak{L}_{1}}
$$

and

$$
\left(u_{1}, \gamma_{1}^{\#}(\lambda)\left(f_{2}-\frac{1}{\lambda} f_{2}^{\prime}\right)\right)_{\mathfrak{L}_{1}}=\left(u_{1}, v_{1}\right) \mathfrak{L}_{1}
$$

This implies

$$
v_{1}=-\frac{1}{\lambda} \gamma_{1}^{\#}(\lambda)\left(f_{2}^{\prime}-\lambda f_{2}\right)
$$

which together with (4.6) yields (4.4).

\subsection{Weyl Function and Spectrum of Proper Extensions of $V$}

A unitary boundary pair $(\mathfrak{L}, \Gamma)$ is a tool which allows to determine those extensions $\widetilde{V}$ of $V$ that satisfy $V \subsetneq \widetilde{V} \subsetneq V_{*}$ in the following way. Let $\Phi$ be a linear relation from $\mathfrak{L}_{1}$ to $\mathfrak{L}_{2}$ represented in the form

$$
\Phi=\left\{\left(\begin{array}{l}
\Phi_{1} h \\
\Phi_{2} h
\end{array}\right): h \in \mathcal{H}\right\}
$$


where $\mathcal{H}$ is an auxiliary Hilbert space and $\Phi_{j}$ are bounded linear operators $\Phi_{j}: \mathcal{H} \rightarrow$ $\mathfrak{L}_{j}(j=1,2)$, such that

$$
\operatorname{ker}\left(\Phi_{1}^{*} \Phi_{1}+\Phi_{2}^{*} \Phi_{2}\right)=\{0\}
$$

Then $\Phi$ is closed if and only if

$$
0 \in \rho\left(\Phi_{1}^{*} \Phi_{1}+\Phi_{2}^{*} \Phi_{2}\right)
$$

Associate with $\Phi$ an extension $V_{\Phi}$ of $V$ by

$$
V_{\Phi}=\left\{\left(\begin{array}{c}
f \\
f^{\prime}
\end{array}\right) \in V_{*}:\left\{\left(\begin{array}{c}
f \\
f^{\prime}
\end{array}\right),\left(\begin{array}{l}
\Phi_{1} h \\
\Phi_{2} h
\end{array}\right)\right\} \in \Gamma \text { for some } h \in \mathcal{H}\right\} .
$$

The following theorem gives a description of the spectrum of $V_{\Phi}$ and contains a Kreŭn type resolvent formula.

Theorem 4.2 Let $V$ be a closed isometric operator in $\mathfrak{H}$, let $\Pi=(\mathfrak{L}, \Gamma)$ be a unitary boundary pair for $V$, let $\Phi \in \mathbf{B}(\mathcal{H}, \mathfrak{L})$ and let (4.8) hold. If $\lambda \in \mathcal{D}$ then:

(i) $\lambda \in \sigma_{p}\left(V_{\Phi}\right) \Longrightarrow \operatorname{ker}\left(\Phi_{2}-\Theta(\lambda) \Phi_{1}\right) \neq\{0\}$;

(ii) $\Phi_{2}-\Theta(\lambda) \Phi_{1}: \mathcal{H} \rightarrow \mathfrak{L}_{2}$ has a bounded inverse $\Longrightarrow \lambda \in \rho\left(V_{\Phi}\right)$.

When (ii) is satisfied the resolvent of $V_{\Phi}$ takes the form

$$
\left(V_{\Phi}-\lambda I_{\mathfrak{H}}\right)^{-1}=\left(V_{1}-\lambda I_{\mathfrak{H}}\right)^{-1}+\frac{1}{\lambda} \gamma_{1}(\lambda) \Phi_{1}\left(\Phi_{2}-\Theta(\lambda) \Phi_{1}\right)^{-1} \gamma_{2}^{\#}(\lambda) \quad(\lambda \in \mathcal{D})
$$

If $\lambda \in \mathcal{D}_{e}$ then:

(iii) $\lambda \in \sigma_{p}\left(V_{\Phi}\right) \Longrightarrow \operatorname{ker}\left(\Phi_{1}-\Theta^{\#}(\lambda) \Phi_{2}\right) \neq\{0\}$;

(iv) $\Phi_{1}-\Theta^{\#}(\lambda) \Phi_{2}: \mathcal{H} \rightarrow \mathfrak{L}_{1}$ has a bounded inverse $\Longrightarrow \lambda \in \rho\left(V_{\Phi}\right)$;

When (iv) is satisfied the resolvent of $V_{\Phi}$ takes the form

$$
\left(V_{\Phi}-\lambda I_{\mathfrak{H}}\right)^{-1}=\left(V_{2}-\lambda I_{\mathfrak{H}}\right)^{-1}-\frac{1}{\lambda} \gamma_{2}(\lambda) \Phi_{2}\left(\Phi_{1}-\Theta^{\#}(\lambda) \Phi_{2}\right)^{-1} \gamma_{1}^{\#}(\lambda) \quad\left(\lambda \in \mathcal{D}_{e}\right)
$$

If, in addition, $\Pi=(\mathfrak{L}, \Gamma)$ is an ordinary boundary triple for $V$ then the implications (i)-(iv) become equivalences.

Proof The proof is divided into steps.

1. Verification of $(i)$. If $\lambda \in \mathcal{D} \cap \sigma_{p}\left(V_{\Phi}\right), \mathcal{D}=\rho\left(V_{1}\right)$, then there is $f \in \mathfrak{H} \backslash\{0\}$ and $h \in \mathcal{H}$ such that

$$
\left\{\left(\begin{array}{c}
f \\
\lambda f
\end{array}\right),\left(\begin{array}{c}
\Phi_{1} h \\
\Phi_{2} h
\end{array}\right)\right\} \in \Gamma
$$


Since $\lambda \in \mathcal{D}$, one has $\lambda \notin \sigma_{p}(V)$ and hence $h \neq 0$. By Definition 3.9 this means that

$$
\Theta(\lambda) \Phi_{1} h=\Phi_{2} h, \quad \lambda \in \mathcal{D}
$$

Hence, $h \in \operatorname{ker}\left(\Phi_{2}-\Theta(\lambda) \Phi_{1}\right)$.

2. Verification of (ii). First assume that $\left(\Phi_{2}-\Theta(\lambda) \Phi_{1}\right)$ has a bounded inverse with $\lambda \in \mathcal{D} \backslash\{0\}$. Let us find a solution $\widehat{f}=\left(\begin{array}{c}f \\ f^{\prime}\end{array}\right) \in V_{\Phi}$ of the equation

$$
f^{\prime}-\lambda f=g
$$

for arbitrary $g \in \mathfrak{H}$. Since $\lambda \in \mathcal{D}=\rho\left(V_{1}\right)$, there are $f_{1}, f_{1}^{\prime} \in \mathfrak{H}$, such that

$$
f_{1}^{\prime}-\lambda f_{1}=g \text { and }\left(\begin{array}{l}
f_{1} \\
f_{1}^{\prime}
\end{array}\right) \in V_{1}
$$

Hence

$$
f_{1}=\left(V_{1}-\lambda I_{\mathfrak{H}}\right)^{-1} g
$$

By Lemma 4.1

$$
\left\{\left(\begin{array}{c}
f_{1} \\
f_{1}^{\prime}
\end{array}\right),\left(\begin{array}{c}
0 \\
u_{2}
\end{array}\right)\right\} \in \Gamma, \text { where } u_{2}=\frac{1}{\lambda} \gamma_{2}^{\#}(\lambda) g=\frac{1}{\lambda} \gamma_{2}^{\#}(\lambda)\left(f_{1}^{\prime}-\lambda f_{1}\right)
$$

Now choose $h=\left(\Phi_{2}-\Theta(\lambda) \Phi_{1}\right)^{-1} u_{2}$ and apply (3.50) to get

$$
\left\{\left(\begin{array}{c}
\gamma_{2}(\lambda) \Phi_{1} h \\
\lambda \gamma_{2}(\lambda) \Phi_{1} h
\end{array}\right),\left(\begin{array}{c}
\Phi_{1} h \\
\Theta(\lambda) \Phi_{1} h
\end{array}\right)\right\} \in \Gamma
$$

Combining (4.17) and (4.18) one obtains

$$
\left\{\left(\begin{array}{l}
f_{1} \\
f_{1}^{\prime}
\end{array}\right)+\left(\begin{array}{c}
\gamma_{1}(\lambda) \Phi_{1} h \\
\lambda \gamma_{1}(\lambda) \Phi_{1} h
\end{array}\right),\left(\begin{array}{c}
\Phi_{1} h \\
u_{2}+\Theta(\lambda) \Phi_{1} h
\end{array}\right)\right\} \in \Gamma
$$

\section{Setting}

$$
\left(\begin{array}{c}
f \\
f^{\prime}
\end{array}\right)=\left(\begin{array}{c}
f_{1} \\
f_{1}^{\prime}
\end{array}\right)+\left(\begin{array}{c}
\left.\gamma_{1}(\lambda)\right) \Phi_{1} h \\
\left.\lambda \gamma_{1}(\lambda)\right) \Phi_{1} h
\end{array}\right)
$$

and using the equality

$$
u_{2}+\Theta(\lambda) \Phi_{1} h=\left(I+\Theta(\lambda) \Phi_{1}\left(\Phi_{2}-\Theta(\lambda) \Phi_{1}\right)^{-1}\right) u_{2}=\Phi_{2} h
$$


one obtains from (4.19)

$$
\left\{\left(\begin{array}{c}
f \\
f^{\prime}
\end{array}\right),\left(\begin{array}{l}
\Phi_{1} h \\
\Phi_{2} h
\end{array}\right)\right\} \in \Gamma
$$

Therefore, the equation (4.15) has a solution $\widehat{f}=\left(\begin{array}{c}f \\ f^{\prime}\end{array}\right) \in V_{\Phi}$ and

$$
\begin{aligned}
f & =f_{1}+\gamma_{1}(\lambda) \Phi_{1} h \\
& =\left(V_{1}-\lambda I_{\mathfrak{H}}\right)^{-1} g+\frac{1}{\lambda} \gamma_{1}(\lambda) \Phi_{1}\left(\Phi_{2}-\Theta(\lambda) \Phi_{1}\right)^{-1} \gamma_{2}^{\#}(\lambda) g
\end{aligned}
$$

Next assume that $0 \in \mathcal{D}$ and $0 \in \rho\left(\Phi_{2}-\Theta(0) \Phi_{1}\right)$. Then by Lemma 4.1 the equality (4.17) holds with $u_{2}=\left(\gamma_{2}^{\#}\right)^{\prime}(0) f_{1}$ and now combining this analog of (4.17) with (4.18) yields (4.21). The formula (4.11) for $\lambda=0$ takes the form

$$
V_{\Phi}^{-1}=V_{1}^{-1}+\gamma_{1}(0) \Phi_{1}\left(\Phi_{2}-\Theta(0) \Phi_{1}\right)^{-1}\left(\gamma_{2}^{\#}\right)^{\prime}(0)
$$

This completes the proof of the implication in (ii) and the formula (4.11).

3. Verification of (iii). If $\lambda \in \sigma_{p}\left(V_{\Phi}\right) \cap \mathcal{D}_{e}$ then there is $f \in \mathfrak{H} \backslash\{0\}$ and $h \in \mathcal{H}$ such that (4.13) holds. Again, since $\lambda \in \mathcal{D}$, one has $\lambda \notin \sigma_{p}(V)$ and hence $h \neq 0$. By Definition 3.9 one gets

$$
\Phi_{1} h-\Theta^{\#}(\lambda) \Phi_{2} h=0
$$

Hence $h \in \operatorname{ker}\left(\Phi_{1}-\Theta^{\#}(\lambda) \Phi_{2}\right)$.

4. Verification of (iv). Assume that $\Phi_{1}-\Theta^{\#}(\lambda) \Phi_{2}$ has a bounded inverse and $\lambda \in \mathcal{D}_{e}$. Since $\lambda \in \mathcal{D}_{e}=\rho\left(V_{2}\right)$, there are $f_{2}, f_{2}^{\prime} \in \mathfrak{H}$, such that

$$
f_{2}^{\prime}-\lambda f_{2}=g \text { and }\left(\begin{array}{l}
f_{2} \\
f_{2}^{\prime}
\end{array}\right) \in V_{2}
$$

Hence $f_{2}=\left(V_{2}-\lambda I_{\mathfrak{H}}\right)^{-1} g$. By Lemma 4.1

$$
\left\{\left(\begin{array}{c}
f_{2} \\
f_{2}^{\prime}
\end{array}\right),\left(\begin{array}{c}
u_{1} \\
0
\end{array}\right)\right\} \in \Gamma \quad \text { with } \quad u_{1}=-\frac{1}{\lambda} \gamma_{1}^{\#}(\lambda) g .
$$

Now choose $h=\left(\Phi_{1}-\Theta^{\#}(\lambda) \Phi_{2}\right)^{-1} u_{1}$ and apply (3.51) to get

$$
\left\{\left(\begin{array}{c}
\gamma_{2}(\lambda) \Phi_{2} h \\
\lambda \gamma_{2}(\lambda) \Phi_{2} h
\end{array}\right),\left(\begin{array}{c}
\Theta^{\#}(\lambda) \Phi_{2} h \\
\Phi_{2} h
\end{array}\right)\right\} \in \Gamma
$$

Combining (4.22) and (4.23) one obtains

$$
\left\{\left(\begin{array}{c}
f \\
f^{\prime}
\end{array}\right),\left(\begin{array}{c}
\Phi_{1} h \\
\Phi_{2} h
\end{array}\right)\right\} \in \Gamma, \text { where }\left(\begin{array}{c}
f \\
f^{\prime}
\end{array}\right)=\left(\begin{array}{c}
f_{2} \\
f_{2}^{\prime}
\end{array}\right)+\left(\begin{array}{c}
\gamma_{2}(\lambda) \Phi_{2} h \\
\lambda \gamma_{2}(\lambda) \Phi_{2} h
\end{array}\right) .
$$


Making use of (4.24), (4.22), and the above formulas for $h$ and $f_{2}$ one obtains

$$
\begin{aligned}
f & =f_{2}+\gamma_{2}(\lambda) \Phi_{2} h \\
& =\left(V_{2}-\lambda I_{\mathfrak{H}}\right)^{-1} g-\frac{1}{\lambda} \gamma_{2}(\lambda) \Phi_{2}\left(\Phi_{1}-\Theta^{\#}(\lambda) \Phi_{2}\right)^{-1} \gamma_{1}^{\#}(\lambda) g .
\end{aligned}
$$

This proves (4.12) and the implication in (iv).

5. Verification of the reverse implication in (i) for the case of an ordinary boundary triple $(\mathfrak{L}, \Gamma)$. Let $\left(\Phi_{2}-\Theta(\lambda) \Phi_{1}\right) h=0$ for some $h \in \mathcal{H} \backslash\{0\}$. Then it follows from

$$
\left\{\left(\begin{array}{c}
\gamma_{1}(\lambda) \Phi_{1} h \\
\lambda \gamma_{1}(\lambda) \Phi_{1} h
\end{array}\right),\left(\begin{array}{c}
\Phi_{1} h \\
\Theta(\lambda) \Phi_{1} h
\end{array}\right)\right\} \in \Gamma
$$

and (4.14) that (4.13) holds with $f=\gamma_{1}(\lambda) \Phi_{1} h$. Notice that $\Phi_{1} h \neq 0$ since otherwise $\Phi_{2} h=0$ by (4.14), which contradicts to (4.8). Therefore, $f \neq 0$ since ker $\gamma_{1}(\lambda)=\{0\}$ for the ordinary boundary triple $\Pi=(\mathfrak{L}, \Gamma)$; see Proposition 3.24. Thus $\lambda \in \sigma_{p}\left(V_{\Phi}\right)$.

6. Verification of the reverse implication in (ii) for the case of an ordinary boundary triple $(\mathfrak{L}, \Gamma)$. Let $\lambda \in \rho\left(V_{\Phi}\right)$. By virtue of item 5 to prove the boundedness of the inverse $\left(\Phi_{2}-\Theta(\lambda) \Phi_{1}\right)^{-1}: \mathfrak{L}_{2} \rightarrow \mathcal{H}$ it is enough to show that

$$
\operatorname{ran}\left(\Phi_{2}-\Theta(\lambda) \Phi_{1}\right)=\mathfrak{L}_{2}
$$

By assumption $\lambda \in \rho\left(V_{\Phi}\right) \cup \mathcal{D}$ and hence for arbitrary $g \in \mathfrak{H}$ one can find vectors $\left(\begin{array}{c}f \\ f^{\prime}\end{array}\right) \in V_{\Phi}$ and $\left(\begin{array}{l}f_{1} \\ f_{1}^{\prime}\end{array}\right) \in V_{1}$ such that

$$
f_{1}^{\prime}-\lambda f_{1}=f-\lambda f^{\prime}=g \quad\left(\lambda \in \rho\left(V_{\Phi}\right) \cup \mathcal{D}\right) .
$$

Then (4.17)-(4.19) hold for some $h \in \mathcal{H}$ and, in particular,

$$
\left(\Phi_{2}-\Theta(\lambda) \Phi_{1}\right) h=u_{2}=\frac{1}{\lambda} \gamma_{2}^{\#}(\lambda) g
$$

Since $g \in \mathfrak{H}$ is arbitrary and for an ordinary boundary triple $\operatorname{ran} \gamma_{2}^{\#}(\lambda)=\mathfrak{L}_{2}$, the claim (4.26) is proved. By Open Mapping Theorem the operator $T:=\Phi_{2}-\Theta(\lambda) \Phi_{1}$ : $\mathcal{H} \rightarrow \mathfrak{L}_{2}$ has a bounded inverse, since $T \in \mathbf{B}\left(\mathcal{H}, \mathfrak{L}_{2}\right)$, ker $T=\{0\}$ and $\operatorname{ran} T=\mathfrak{L}_{2}$.

The operator function $\left(I_{\mathfrak{H}}-z V_{\Phi}\right)^{-1}$ is called the coresolvent of $V_{\Phi}$. Setting $\lambda=1 / z$ in Theorem 4.2 one obtains the following statement for coresolvents of $V_{\Phi}$.

Corollary 4.3 Let $V$ be a closed isometric operator in $\mathfrak{H}$, let $\Pi=(\mathfrak{L}, \Gamma)$ be a unitary boundary pair for $V$, let $\Phi \in \mathbf{B}(\mathcal{H}, \mathfrak{L})$ and let (4.8) hold. If $z \in \overline{\mathcal{D}}$ then:

(i) $z \in \sigma_{p}\left(V_{\Phi}^{-1}\right) \Longrightarrow \operatorname{ker}\left(\Phi_{1}-\Theta(\bar{z})^{*} \Phi_{2}\right) \neq\{0\}$;

(ii) $\Phi_{1}-\Theta(\bar{z})^{*} \Phi_{2}: \mathcal{H} \rightarrow \mathfrak{L}_{1}$ has a bounded inverse $\Longrightarrow z \in \rho\left(V_{\Phi}^{-1}\right)$. 
When (ii) is satisfied then the coresolvent of $V_{\Phi}$ takes the form

$$
\left(I_{\mathfrak{H}}-z V_{\Phi}\right)^{-1}=\left(I_{\mathfrak{H}}-z V_{2}\right)^{-1}+\gamma_{2}\left(\frac{1}{z}\right) \Phi_{2}\left(\Phi_{1}-\Theta(\bar{z})^{*} \Phi_{2}\right)^{-1} \gamma_{1}(\bar{z})^{*}
$$

If $z \in \overline{\mathcal{D}_{e}}$ then:

(iii) $z \in \sigma_{p}\left(V_{\Phi}^{-1}\right) \Longrightarrow \operatorname{ker}\left(\Phi_{2}-\Theta(1 / z) \Phi_{1}\right) \neq\{0\}$;

(iv) $\Phi_{2}-\Theta(1 / z) \Phi_{1}: \mathcal{H} \rightarrow \mathfrak{L}_{2}$ has a bounded inverse $\Longrightarrow z \in \rho\left(V_{\Phi}^{-1}\right)$.

When (iv) is satisfied then the coresolvent of $V_{\Phi}$ takes the form

$$
\left(I_{\mathfrak{H}}-z V_{\Phi}\right)^{-1}=\left(I_{\mathfrak{H}}-z V_{1}\right)^{-1}-\gamma_{1}(1 / z) \Phi_{1}\left(\Phi_{2}-\Theta(1 / z) \Phi_{1}\right)^{-1} \gamma_{2}(\bar{z})^{*}
$$

If, in addition, $\Pi=(\mathfrak{L}, \Gamma)$ is an ordinary boundary triple for $V$ then the implications (i)-(iv) become equivalences.

Remark 4.4 If $\left(\mathfrak{L}_{1} \oplus \mathfrak{L}_{2}, \Gamma_{1}, \Gamma_{2}\right)$ is an ordinary boundary triple for $V$ then every closed proper extension of $V$ can be represented in the form (4.10) with $\Phi_{j} \in \mathbf{B}\left(\mathcal{H}, \mathfrak{L}_{j}\right)$ $(j=1,2)$ such that (4.9) holds due to [5, Theorem 2.1], [27, Proposition 6.12]. Moreover, if $\Phi$ is defined by (4.7), then the following equivalences hold:

(1) $V_{\Phi}$ is an isometric relation in $\mathcal{H} \Longleftrightarrow \Phi$ is the graph of an isometric operator;

(2) $V_{\Phi}$ is a unitary relation in $\mathcal{H} \Longleftrightarrow \Phi$ is the graph of a unitary operator;

(3) $V_{\Phi}$ is a contractive relation in $\mathcal{H} \Longleftrightarrow \Phi$ is the graph of a contraction.

The fact that the implications (i)-(iv) of Theorem 4.2 become equivalences for an ordinary boundary triple $\Pi=(\mathfrak{L}, \Gamma)=\left(\mathfrak{L}, \Gamma_{1}, \Gamma_{2}\right)$ was proved in [41] in the case when $\kappa=0$, and in [5] in the case $\kappa \neq 0$.

\subsection{Description of Generalized Coresolvents}

Definition 4.5 (see [38,39]) An operator-valued function $\mathbf{K}_{\lambda}$ holomorphic in a domain $\mathcal{O} \subseteq \mathbb{D}$ with values in $\mathbf{B}(\mathfrak{H})$ is called a generalized coresolvent of an isometric operator $V: \mathfrak{H} \rightarrow \mathfrak{H}$, if there exist a Pontryagin space $\widetilde{\mathfrak{H}} \supset \mathfrak{H}$ with negative index $\widetilde{\kappa}=\kappa_{-}(\widetilde{\mathfrak{H}})$ and a unitary extension $\widetilde{V}: \widetilde{\mathfrak{H}} \rightarrow \widetilde{\mathfrak{H}}$ of the operator $V$ such that $\mathcal{O} \subseteq \rho\left(\widetilde{V}^{-1}\right)$, and

$$
\mathbf{K}_{z}=P_{\mathfrak{H}}\left(I_{\tilde{H}}-z \widetilde{V}\right)^{-1}\lceil\mathfrak{H}, \quad z \in \mathcal{O},
$$

where $P_{\mathfrak{H}}$ is the orthogonal projection from $\widetilde{\mathfrak{H}}$ onto $\mathfrak{H}$. Notice that in [38] the operator function $\mathbf{K}_{z}$ in (4.29) is called a generalized resolvent of $V$.

The representation (4.29) of the generalized coresolvent of $V$ is called minimal, if

$$
\widetilde{\mathfrak{H}}=\overline{\operatorname{span}}\left\{\mathfrak{H}+\left(I_{\widetilde{H}}-z \widetilde{V}\right)^{-1} \mathfrak{H}: \lambda \in \mathcal{O}\right\}
$$


The generalized coresolvent $\mathbf{K}_{z}$ is said to be $k$-regular, if $k=\kappa_{-}(\widetilde{\mathfrak{H}}[-] \mathfrak{H})$ for a minimal representation (4.29).

Every generalized coresolvent $\mathbf{K}_{z}$ of the isometric operator $V$ admits a minimal representation (4.29) and every two minimal representations of $\mathbf{K}_{z}$ are unitarily equivalent, see [14, Proposition 4.1] for the case of a symmetric operator.

Lemma 4.6 Let $\mathbf{K}_{z}$ be a $(\widetilde{\kappa}-\kappa)$-regular generalized coresolvent. Then the kernel

$$
R_{w}(z):=\frac{\mathbf{K}_{z}+\mathbf{K}_{w}^{[*]}-I}{1-z \bar{w}}-\mathbf{K}_{w}^{[*]} \mathbf{K}_{z}
$$

has $\widetilde{\kappa}-\kappa$ negative squares on $\rho\left(\widetilde{V}^{-1}\right) \cap \mathbb{D}$.

Proof Let $\left\{z_{j}\right\}_{j=1}^{n}$ be a set of points in $\rho\left(\widetilde{V}^{-1}\right) \cap \mathbb{D}$ and let $g_{j} \in \mathfrak{H}, j=1,2, \ldots, n$. Denote

$$
\widetilde{f}_{j}:=\left(I_{\widetilde{H}}-z_{j} \widetilde{V}\right)^{-1} g_{j}, \quad f_{j}:=P_{\mathfrak{H}} \widetilde{f}_{j}=\mathbf{K}_{z_{j}} g_{j}, \quad(j=1,2, \ldots, n)
$$

Then it follows form the first equality in (4.31) that

$$
\left(\begin{array}{c}
\tilde{f}_{j} \\
g_{j}
\end{array}\right) \in I_{\tilde{H}}-z_{j} \tilde{V} \Longleftrightarrow\left(\begin{array}{c}
z_{j} \tilde{f}_{j} \\
\tilde{f}_{j}-g_{j}
\end{array}\right) \in \widetilde{V} .
$$

Since $\widetilde{V}$ is a unitary relation in $\widetilde{\mathfrak{H}}$ one obtains $z_{j} \bar{z}_{k}\left[\widetilde{f}_{j}, \widetilde{f}_{k}\right] \widetilde{\mathfrak{H}}=\left[\widetilde{f}_{j}-g_{j}, \widetilde{f}_{k}-g_{k}\right] \widetilde{\mathfrak{H}}$ or, equivalently,

$$
\left(1-z_{j} \bar{z}_{k}\right)\left[\tilde{f}_{j}, \tilde{f}_{k}\right]_{\mathfrak{H}}=\left[\tilde{f}_{j}, g_{k}\right]_{\tilde{H}}+\left[g_{j}, \widetilde{f}_{k}\right]_{\tilde{H}}-\left[g_{j}, g_{k}\right]_{\mathfrak{H}} .
$$

It follows from (4.30), (4.31), and (4.32) that

$$
\begin{aligned}
& \sum_{j, k=1}^{n}\left[\mathrm{R}_{z_{k}}\left(z_{j}\right) g_{j}, g_{k}\right]_{\mathfrak{H}} \xi_{j} \bar{\xi}_{k}=-\sum_{j, k=1}^{n}\left[\mathbf{K}_{z_{j}} g_{j}, \mathbf{K}_{z_{k}} g_{k}\right]_{\mathfrak{H}} \xi_{j} \bar{\xi}_{k} \\
& +\sum_{j, k=1}^{n} \frac{\left[\mathbf{K}_{z_{j}} g_{j}, g_{k}\right]_{\mathfrak{H}}+\left[g_{j}, \mathbf{K}_{z_{k}} g_{k}\right]_{\mathfrak{H}}-\left[g_{j}, g_{k}\right]_{\mathfrak{H}}}{1-z_{j} \bar{z}_{k}} \xi_{j} \bar{\xi}_{k} \\
& (4.31) \sum_{j, k=1}^{n}\left\{\frac{\left[\tilde{f}_{j}, g_{k}\right]_{\mathfrak{H}}+\left[g_{j}, \tilde{f}_{k}\right]_{\mathfrak{H}}-\left[g_{j}, g_{k}\right]_{\mathfrak{H}}}{1-z_{j} \bar{z}_{k}}-\left[f_{j}, f_{k}\right]_{\mathfrak{H}}\right\} \xi_{j} \bar{\xi}_{k} \\
& \stackrel{(4.32)}{=} \sum_{j, k=1}^{n}\left\{\left[\tilde{f}_{j}, \widetilde{f}_{k}\right]_{\tilde{H}}-\left[f_{j}, f_{k}\right]_{\mathfrak{H}}\right\} \xi_{j} \bar{\xi}_{k} \\
& =\sum_{j, k=1}^{n}\left[\left(I-P_{\mathfrak{H}}\right) \tilde{f}_{j},\left(I-P_{\mathfrak{H}}\right) \widetilde{f}_{k}\right]_{\mathfrak{H}} \xi_{j} \bar{\xi}_{k}
\end{aligned}
$$


This form has at most $\widetilde{\kappa}-\kappa$ negative squares, since ind $(\widetilde{\mathfrak{H}}[-] \mathfrak{H})=\widetilde{\kappa}-\kappa$. Because the representation $(4.29)$ is $(\widetilde{\kappa}-\kappa)$-regular the set

$$
\left\{\left(I_{\widetilde{H}}-P_{\mathfrak{H}}\right)\left(I_{\widetilde{H}}-z \widetilde{V}\right)^{-1} \mathfrak{H}: z \in \rho\left(\widetilde{V}^{-1}\right)\right\}
$$

is dense in $\widetilde{\mathfrak{H}}[-] \mathfrak{H}$ and hence it contains a $(\widetilde{\kappa}-\kappa)$-dimensional negative subspace. Therefore, the form (4.33) has exactly $\widetilde{\kappa}-\kappa$ negative squares for an appropriate choice of $z_{j}, g_{j}(j=1,2, \ldots, n)$.

Remark 4.7 In the case of a standard isometric operator the statement of Lemma 4.6 was proved in [28].

Theorem 4.8 Let $V: \mathfrak{H} \rightarrow \mathfrak{H}$ be an isometric operator, let $\left(\mathfrak{L}_{1} \oplus \mathfrak{L}_{2}, \Gamma_{1}, \Gamma_{2}\right)$ be an ordinary boundary triple for $V$, and let $\Theta(\cdot), \gamma_{1}(\cdot), \gamma_{2}(\cdot)$ be the corresponding Weyl function and the $\gamma$-fields.

Then for $z \in \overline{\mathcal{D}} \cap \rho\left(\tilde{V}^{-1}\right)$ the formula

$$
\mathbf{K}_{z}=\left(I_{\widetilde{H}}-z V_{2}\right)^{-1}+\gamma_{2}(1 / z) \varepsilon(z)\left(I_{\mathfrak{L}_{1}}-\Theta(\bar{z})^{*} \varepsilon(z)\right)^{-1} \gamma_{1}(\bar{z})^{*}
$$

establishes a one-to-one correspondence between the set of $\widetilde{\kappa}-\kappa$-regular generalized coresolvents of $V$ and the set of all operator-valued functions $\varepsilon(\cdot) \in \mathcal{S}_{\widetilde{\kappa}-\kappa}\left(\mathfrak{L}_{1}, \mathfrak{L}_{2}\right)$, such that

$$
0 \in \rho\left(I_{\mathfrak{L}_{1}}-\Theta(\bar{z})^{*} \varepsilon(z)\right)
$$

For $z \in \overline{\mathcal{D}}_{e} \cap \rho\left(\widetilde{V}^{-1}\right)$ the formula (4.34) takes the form

$$
\mathbf{K}_{z}=\left(I_{\tilde{H}}-z V_{1}\right)^{-1}-\gamma_{1}(1 / z) \varepsilon^{T}(1 / z)\left(I_{\mathfrak{L}_{2}}-\Theta(1 / z) \varepsilon^{T}(1 / z)\right)^{-1} \gamma_{2}(\bar{z})^{*}
$$

Proof The proof is divided into steps.

1. Verification that for every $\varepsilon(\cdot) \in \mathcal{S}_{\widetilde{\kappa}-\kappa}\left(\mathfrak{L}_{1}, \mathfrak{L}_{2}\right)$ satisfying (4.35) the formula (4.34) determines a $(\widetilde{\kappa}-\kappa)$-regular generalized coresolvent of $V$.

By Theorem 3.17 there exists a simple isometric operator $V^{-}$in a Pontryagin space $\mathfrak{H}^{-}$with negative index $\widetilde{\kappa}-\kappa$ and a unitary boundary pair $\left(\mathfrak{L}, \Gamma^{-}\right)$such that the corresponding Weyl function $\Theta^{-}(z)$ coincides with $\varepsilon(z)$ for $z \in \mathfrak{h}_{\varepsilon}=\rho\left(V_{1}^{-}\right)$, where $V_{1}^{-}=\operatorname{ker} \Gamma_{1}^{-}$.

Next we construct a new unitary boundary pair $(\widetilde{\mathfrak{L}}, \widetilde{\Gamma})$ as the direct sum of the ordinary boundary triple $\left(\mathfrak{L}, \Gamma^{+}\right):=(\mathfrak{L}, \Gamma)$ and the unitary boundary pair $\left(\mathfrak{L}, \Gamma^{-}\right)$by 
the formulas

$$
\begin{aligned}
& \widetilde{\mathfrak{L}}=\widetilde{\mathfrak{L}}_{1} \oplus \widetilde{\mathfrak{L}}_{2}, \text { where } \widetilde{\mathfrak{L}}_{1}=\widetilde{\mathfrak{L}}_{2}=\left(\begin{array}{l}
\mathfrak{L}_{1} \\
\mathfrak{L}_{2}
\end{array}\right), \\
& \widetilde{\Gamma}=\left\{\left\{\left(\begin{array}{l}
f_{+} \\
f_{-} \\
f_{+}^{\prime} \\
f_{-}^{\prime}
\end{array}\right),\left(\begin{array}{l}
u_{1}^{+} \\
u_{2}^{-} \\
u_{1}^{-} \\
u_{2}^{+}
\end{array}\right)\right\}:\left\{\left(\begin{array}{l}
f_{ \pm} \\
f_{ \pm}^{\prime}
\end{array}\right),\left(\begin{array}{l}
u_{1}^{ \pm} \\
u_{2}^{ \pm}
\end{array}\right)\right\} \in \Gamma^{ \pm}\right\} .
\end{aligned}
$$

Let $\varepsilon(z), \gamma_{1}^{-}(z), \gamma_{2}^{-}(z)$ be the Weyl function and the $\gamma$-fields of the unitary boundary pair $\left(\mathfrak{L}, \Gamma^{-}\right)$, let $\varepsilon^{T}(z), \gamma_{1}^{-, T}(z), \gamma_{2}^{-, T}(z)$ be the Weyl function and the $\gamma$-fields of the transposed boundary pair $\left(\mathfrak{L},\left(\Gamma^{-}\right)^{T}\right)$ and let $\widetilde{V}_{1}=\operatorname{ker} \widetilde{\Gamma}_{1}, \widetilde{V}_{2}=\operatorname{ker} \widetilde{\Gamma}_{2}$. Then the Weyl function $\widetilde{\Theta}(z)$ and the $\gamma$-fields $\widetilde{\gamma}_{1}(z), \widetilde{\gamma}_{2}(z)$ of the unitary boundary pair $(\widetilde{\mathfrak{L}}, \widetilde{\Gamma})$ are given by

$$
\begin{aligned}
& \widetilde{\Theta}(z)=\left(\begin{array}{cc}
0 & \varepsilon^{T}(z) \\
\Theta(z) & 0
\end{array}\right):\left(\begin{array}{l}
\mathfrak{L}_{1} \\
\mathfrak{L}_{2}
\end{array}\right) \rightarrow\left(\begin{array}{l}
\mathfrak{L}_{1} \\
\mathfrak{L}_{2}
\end{array}\right), \quad z \in \widetilde{\mathcal{D}}:=\rho\left(\widetilde{V}_{1}\right) \cap \mathbb{D}, \\
& \tilde{\gamma}_{2}(z):=\left(\begin{array}{cc}
0 & \gamma_{2}^{+}(z) \\
\gamma_{2}^{-, T}(z) & 0
\end{array}\right):\left(\begin{array}{l}
\mathfrak{L}_{1} \\
\mathfrak{L}_{2}
\end{array}\right) \rightarrow\left(\begin{array}{c}
\mathfrak{H} \\
\mathfrak{H}^{-}
\end{array}\right), \quad z \in \widetilde{\mathcal{D}}=\rho\left(\widetilde{V}_{1}\right) \cap \mathbb{D}, \quad \\
& \widetilde{\gamma}_{1}(z)=\left(\begin{array}{cc}
\gamma_{1}^{+}(z) & 0 \\
0 & \gamma_{1}^{-, T}(z)
\end{array}\right):\left(\begin{array}{c}
\mathfrak{L}_{1} \\
\mathfrak{L}_{2}
\end{array}\right) \rightarrow\left(\begin{array}{c}
\mathfrak{H} \\
\mathfrak{H}^{-}
\end{array}\right), \quad z \in \widetilde{\mathcal{D}}_{e}:=\rho\left(\widetilde{V}_{2}\right) \cap \mathbb{D}_{e} .
\end{aligned}
$$

Consider the extension $\widetilde{V}_{\Phi}$ of the operator $\widetilde{V}=\left(\begin{array}{cc}V & 0 \\ 0 & V^{-}\end{array}\right)$in the space $\widetilde{\mathfrak{H}}:=$ $\mathfrak{H}^{+} \oplus \mathfrak{H}^{-}, \mathfrak{H}^{+}:=\mathfrak{H}$, corresponding to the linear relation $\Phi$ of the form (4.7), where

$$
\Phi_{1}=\Phi_{2}=I_{\mathfrak{L}_{1} \oplus \mathfrak{L}_{2}}
$$

In view of (4.37) the extension $\widetilde{V}_{\Phi}$ takes the form

$$
\widetilde{V}_{\Phi}=\left\{\left\{\left(\begin{array}{c}
f_{+} \\
f_{-}
\end{array}\right),\left(\begin{array}{l}
f_{+}^{\prime} \\
f_{-}^{\prime}
\end{array}\right)\right\}:\left\{\left(\begin{array}{c}
f_{-} \\
f_{-}^{\prime}
\end{array}\right),\left(\begin{array}{l}
\Gamma_{2} \widehat{f_{+}} \\
\Gamma_{1} \widehat{f}_{+}
\end{array}\right)\right\} \in \Gamma^{-}, \widehat{f_{+}} \in V^{-[*]}\right\}
$$

Then $I_{\widetilde{\mathfrak{L}}_{1}}-\widetilde{\Theta}(\bar{z})^{*}=\left(\begin{array}{cc}I_{\mathfrak{L}_{1}} & -\Theta(\bar{z})^{*} \\ -\varepsilon(z) & I_{\mathfrak{L}_{2}}\end{array}\right)$ and the assumption (4.35) yields

$$
0 \in \rho\left(I_{\widetilde{\mathfrak{L}}_{1}}-\widetilde{\Theta}(\bar{z})^{*}\right)
$$

for $\bar{z} \in \widetilde{\mathcal{D}}$. By (4.41) and Corollary 4.3 one obtains $z \in \rho\left(\widetilde{V}_{\Phi}^{-1}\right)$ and

$$
\left(I_{\widetilde{H}}-z \widetilde{V}_{\Phi}\right)^{-1}=\left(I_{\tilde{H}}-z \widetilde{V}_{2}\right)^{-1}+\widetilde{\gamma}_{2}(1 / z)\left(I_{\widetilde{\mathfrak{L}}_{1}}-\widetilde{\Theta}(\bar{z})^{*}\right)^{-1} \widetilde{\gamma}_{1}(\bar{z})^{*}
$$


By using (4.40), (4.39), (4.42) and the formula (see [10][Prop. 2.8.7, p.108])

$$
\left(I_{\widetilde{L}_{1}}-\widetilde{\Theta}(\bar{z})^{*}\right)^{-1}=\left(\begin{array}{cc}
I_{\mathfrak{L}_{1}} & -\Theta(\bar{z})^{*} \\
-\varepsilon(z) & I_{\mathfrak{L}_{2}}
\end{array}\right)^{-1}=\left(\begin{array}{cc}
* & * \\
\varepsilon(z)\left(I_{\mathfrak{L}_{1}}-\Theta(\bar{z})^{*} \varepsilon(z)\right)^{-1} & *
\end{array}\right)
$$

one obtains the equality

$$
\begin{aligned}
& \left(I_{\tilde{H}}-z \widetilde{V}_{\Phi}\right)^{-1}=\left(I_{\widetilde{H}}-z \widetilde{V}_{2}\right)^{-1} \\
& \quad+\left(\begin{array}{cc}
0 & \gamma_{2}(z) \\
\gamma_{2}^{-, T}(z) & 0
\end{array}\right)\left(\begin{array}{cc}
* & * \\
\varepsilon(z)\left(I_{\mathfrak{L}_{1}}-\Theta(\bar{z})^{*} \varepsilon(z)\right)^{-1} & *
\end{array}\right)\left(\begin{array}{cc}
\gamma_{1}(\bar{z})^{*} & 0 \\
0 & \gamma_{1}^{-, T}(\bar{z})^{*}
\end{array}\right)
\end{aligned}
$$

where $*$ denotes blocks which are not used in further calculations. Considering the compression of the formula (4.44) to the subspace $\mathfrak{H}^{+}=\mathfrak{H}$ one arrives at (4.34).

Similarly, for $\bar{z} \in \widetilde{\mathcal{D}}_{e}$ one obtains by (4.41) and Corollary 4.3 that $z \in \rho\left(\widetilde{V}_{\Phi}^{-1}\right)$ and

$$
\left(I_{\tilde{\mathfrak{H}}}-z \widetilde{V}_{\Phi}\right)^{-1}=\left(I_{\tilde{\mathfrak{H}}}-z \widetilde{V}_{1}\right)^{-1}-\widetilde{\gamma}_{1}(1 / z)\left(I_{\widetilde{\mathfrak{L}}_{1}}-\widetilde{\Theta}(1 / z)\right)^{-1} \widetilde{\gamma}_{2}(\bar{z})^{*}
$$

By (4.43)

$$
\left(I_{\widetilde{\mathfrak{L}}_{1}}-\widetilde{\Theta}\left(\frac{1}{z}\right)\right)^{-1}=\left(\begin{array}{cc}
* \omega_{12}(z) \\
* & *
\end{array}\right)
$$

where $\omega_{12}(z)=\varepsilon^{T}(1 / z)\left(I_{\mathfrak{L}_{2}}-\Theta(1 / z) \varepsilon^{T}(1 / z)\right)^{-1}$. Using (4.40), (4.39) and (4.45) one gets

$$
\begin{aligned}
& \left(I_{\widetilde{H}}-z \widetilde{V}_{\Phi}\right)^{-1}=\left(I_{\widetilde{H}}-z \widetilde{V}_{1}\right)^{-1} \\
& \quad-\left(\begin{array}{cc}
\gamma_{1}(1 / z) & 0 \\
0 & \gamma_{1}^{-, T}(1 / z)
\end{array}\right)\left(\begin{array}{cc}
* \omega_{12}(z) \\
* & *
\end{array}\right)\left(\begin{array}{cc}
0 & \gamma_{2}^{-, T}(\bar{z})^{*} \\
\gamma_{2}(\bar{z})^{*} & 0
\end{array}\right) .
\end{aligned}
$$

The compression of the formula (4.46) to the subspace $\mathfrak{H}^{+}$gives the equality (4.36).

2. Verification that every $(\widetilde{\kappa}-\kappa)$ - regular generalized coresolvent of $V$ admits the representation (4.34), where $\varepsilon(\cdot) \in \mathcal{S}_{\widetilde{\kappa}-\kappa}\left(\mathfrak{L}_{1}, \mathfrak{L}_{2}\right)$ and $(4.35)$ holds.

First observe that for $z \in \rho\left(\widetilde{V}^{-1}\right)$ and $g \in \mathfrak{H}$ the following relations hold:

$$
\left(\begin{array}{c}
z\left(I_{\widetilde{H}}-z \widetilde{V}\right)^{-1} g \\
\left(I_{\widetilde{H}}-z \widetilde{V}\right)^{-1} g-g
\end{array}\right) \in \widetilde{V}, \quad \widehat{\mathbf{K}}_{z} g:=\left(\begin{array}{c}
z \mathbf{K}_{z} g \\
\mathbf{K}_{z} g-g
\end{array}\right) \in V^{-[*]}
$$

Indeed, the first relation in (4.47) is self-evident and hence for every $h \in \operatorname{dom} V$ one also has the equality

$$
\left[z\left(I_{\widetilde{H}}-z \widetilde{V}\right)^{-1} g, h\right]_{\mathfrak{H}}=\left[-g+\left(I_{\mathfrak{H}}-z \widetilde{V}\right)^{-1} g, V h\right]_{\mathfrak{H}}
$$


On the other hand,

$$
\begin{aligned}
{\left[z \mathbf{K}_{z} g, h\right]_{\mathfrak{H}} } & +\left[\left(I_{\mathfrak{H}}-\mathbf{K}_{z}\right) g, V h\right]_{\mathfrak{H}}=\left[\mathbf{K}_{z} g,\left(\bar{z} I_{\mathfrak{H}}-V\right) h\right]_{\mathfrak{H}}+[g, V h]_{\mathfrak{H}} \\
& =\left[\left(I_{\widetilde{H}}-z \widetilde{V}\right)^{-1} g,\left(\bar{z} I_{\mathfrak{H}}-V\right) h\right]_{\mathfrak{H}}+[g, V h]_{\mathfrak{H}} \\
& =\left[z\left(I_{\widetilde{H}}-z \widetilde{V}\right)^{-1} g, h\right]_{\mathfrak{H}}-\left[\left(I_{\widetilde{H}}-z \widetilde{V}\right)^{-1} g, V h\right]_{\mathfrak{H}}+[g, V h]_{\mathfrak{H}}=0,
\end{aligned}
$$

and here the last equality follows from (4.48). This proves the second relation in (4.47).

Next consider the linear relation

$$
V_{z}:=\left\{\widehat{\mathbf{K}}_{z} g: g \in \mathfrak{H}\right\}, \quad z \in \rho\left(\widetilde{V}^{-1}\right)
$$

in $\mathfrak{H}$. The linear relation $V_{z}$ is closed, since the assumptions

$$
z \mathbf{K}_{z} g_{n} \rightarrow f, \quad \mathbf{K}_{z} g_{n}-g_{n} \rightarrow f^{\prime} \quad(n \rightarrow \infty)
$$

imply $g_{n} \rightarrow g:=\frac{1}{z}\left(f-z f^{\prime}\right)(z \neq 0)$ and hence

$$
\left(\begin{array}{c}
f \\
f^{\prime}
\end{array}\right)=\left(\begin{array}{c}
z \mathbf{K}_{z} g \\
\mathbf{K}_{z} g-g
\end{array}\right) \in V_{z} .
$$

In order to construct a parametric representation of the proper extension $V_{z}$ let us introduce a closed subspace $\mathcal{N}$ of $V_{2}$ such that

$$
V_{2}=V \dot{+} \mathcal{N}
$$

Since $V^{-[*]}=V_{1}+V_{2}$, this implies

$$
V^{-[*]}=V_{1}+\mathcal{N}
$$

Let $P_{1}$ and $P_{2}$ be projections onto the 1-st and the 2-nd components in $\mathfrak{H}^{2}$ and let $\Phi_{j}(z)$ be operator functions with values in $\mathbf{B}\left(\mathcal{N}, \mathfrak{L}_{j}\right)$ defined by

$$
\Phi_{j}(z) h=\Gamma_{j} \widehat{\mathbf{K}}_{z}\left(P_{1} h-z P_{2} h\right), \quad h \in \mathcal{N}, \quad z \in \rho\left(\widetilde{V}^{-1}\right), \quad j=1,2 .
$$

The values of $\Phi_{j}(z)$ belong to $\mathbf{B}\left(\mathcal{N}, \mathfrak{L}_{j}\right)$ due to formula (4.47). Moreover, $\Phi_{1}(z)$ and $\Phi_{2}(z)$ satisfy (4.8), since the assumption $\Phi_{1}(z) h=\Phi_{2}(z) h=0$ implies $h \in V \cap \mathcal{N}=$ $\{0\}$.

Now introduce the linear relation

$$
\varepsilon(z):=\left\{\left(\begin{array}{l}
\Phi_{1}(z) h \\
\Phi_{2}(z) h
\end{array}\right): h \in \mathcal{N}\right\} \quad z \in \rho\left(\widetilde{V}^{-1}\right) .
$$

Since $\operatorname{ran}\left(I_{\mathfrak{H}}-z V_{2}\right)=\mathfrak{H}$ for all $z \in \mathcal{D}$ it follows from (4.49) and (4.51) that the linear relations $V_{z}$ and $\varepsilon(z)$ are connected via (4.10) and hence

$$
V_{z}=V_{\varepsilon(z)}, \quad z \in \rho\left(\widetilde{V}^{-1}\right) .
$$


Since $(\mathfrak{L}, \Gamma)$ is an ordinary boundary triple one concludes that $\varepsilon(z)$ is closed in $\mathfrak{L}_{1} \oplus \mathfrak{L}_{2}$, and hence by Remark $4.4 \Phi_{1}(z)$ and $\Phi_{2}(z)$ satisfy (4.9); cf. [5, Theorem 2.1]. Using (4.47) and (4.52) one obtains

$$
\mathbf{K}_{z} g=\left(I_{\mathfrak{H}}-z V_{\varepsilon(z)}\right)^{-1} g, \quad g \in \mathfrak{H} .
$$

Therefore $z \in \rho\left(V_{\varepsilon(z)}^{-1}\right)$ for all $z \in \rho\left(\widetilde{V}^{-1}\right)$ and since $(\mathfrak{L}, \Gamma)$ is an ordinary boundary triple Corollary 4.3 shows that $0 \in \rho\left(I_{\mathfrak{L}_{1}}-\Theta(z) \varepsilon(z)\right)$ and that the following formula holds for $z \in \rho\left(\widetilde{V}^{-1}\right) \cap \overline{\mathcal{D}}$ :

$$
\left(I_{\mathfrak{H}}-z V_{\varepsilon(z)}\right)^{-1}=\left(I_{\mathfrak{H}}-z V_{2}\right)^{-1}+\gamma_{2}(1 / z) \Phi_{2}(z)\left(\Phi_{1}(z)-\Theta(\bar{z})^{*} \Phi_{2}(z)\right)^{-1} \gamma_{1}(\bar{z})^{*}
$$

It remains to show that $\varepsilon(\cdot) \in \mathcal{S}_{\widetilde{\kappa}-\kappa}\left(\mathfrak{L}_{1}, \mathfrak{L}_{2}\right)$. For a choice of $z_{j} \in \rho\left(\widetilde{V}^{-1}\right) \cap \mathbb{D}$ and $g_{j} \in \mathfrak{H}$ denote

$$
\widehat{f_{j}}=\left(\begin{array}{c}
f_{j} \\
f_{j}^{\prime}
\end{array}\right):=\widehat{\mathbf{K}}_{z_{j}} g_{j}, \quad j=1, \ldots, n
$$

and let

$$
\Gamma \widehat{f_{j}}=\left(\begin{array}{l}
\Phi_{1}\left(z_{j}\right) h_{j} \\
\Phi_{2}\left(z_{j}\right) h_{j}
\end{array}\right), \quad h_{j} \in \mathcal{N}, \quad j=1, \ldots, n .
$$

Then it follows from (4.55), (4.56) and (3.2) that with $\xi_{j} \in \mathbb{C}$,

$$
\begin{aligned}
\sum_{j, k=1}^{n} a_{j, k} \xi_{j} \overline{\xi_{k}} & :=\sum_{j, k=1}^{n} \frac{\left(\Phi_{1}\left(z_{j}\right) h_{j}, \Phi_{1}\left(z_{k}\right) h_{k}\right) \mathfrak{L}_{1}-\left(\Phi_{2}\left(z_{j}\right) h_{j}, \Phi_{2}\left(z_{k}\right) h_{k}\right) \mathfrak{L}_{2}}{1-z_{j} \bar{z}_{k}} \xi_{j} \overline{\xi_{k}} \\
& =\sum_{j, k=1}^{n} \frac{\left(\Gamma_{1} \widehat{f_{j}}, \Gamma_{1} \widehat{f_{k}}\right) \mathfrak{L}_{1}-\left(\Gamma_{2} \widehat{f_{j}}, \Gamma_{2} \widehat{f}_{k}\right) \mathfrak{L}_{2}}{1-z_{j} \bar{z}_{k}} \xi_{j} \xi_{k} \\
& =\sum_{j, k=1}^{n}\left\{\left[f_{j}, f_{k}\right]_{\mathfrak{H}}-\left[f_{j}^{\prime}, f_{k}^{\prime}\right]_{\mathfrak{H}}\right\} \frac{\xi_{j} \bar{\xi}_{k}}{1-z_{j} \bar{z}_{k}} .
\end{aligned}
$$

Since

$$
\begin{aligned}
& {\left[f_{j}, f_{k}\right]_{\mathfrak{H}}-\left[f_{j}^{\prime}, f_{k}^{\prime}\right]_{\mathfrak{H}}=z_{j} \bar{z}_{k}\left[\mathbf{K}_{z_{j}} g_{j}, \mathbf{K}_{z_{k}} g_{k}\right]_{\mathfrak{H}}-\left[\mathbf{K}_{z_{j}} g_{j}-g_{j}, \mathbf{K}_{z_{k}} g_{k}-g_{k}\right]_{\mathfrak{H}}} \\
& \quad=\left(z_{j} \bar{z}_{k}-1\right)\left[\mathbf{K}_{z_{j}} g_{j}, \mathbf{K}_{z_{k}} g_{k}\right]_{\mathfrak{H}}+\left[\mathbf{K}_{z_{j}} g_{j}, g_{k}\right]_{\mathfrak{H}}+\left[g_{j}, \mathbf{K}_{z_{k}} g_{k}\right]_{\mathfrak{H}}-\left[g_{j}, g_{k}\right]_{\mathfrak{H}} \\
& \quad=\left(1-z_{j} \bar{z}_{k}\right)\left[\mathbf{R}_{z_{k}}\left(z_{j}\right) g_{j}, g_{k}\right]_{\mathfrak{H}}
\end{aligned}
$$

the form in (4.57) is reduced to

$$
\sum_{j, k=1}^{n} a_{j, k} \xi_{j} \overline{\xi_{k}}=\sum_{j, k=1}^{n}\left[\mathrm{R}_{z_{k}}\left(z_{j}\right) g_{j}, g_{k}\right]_{\mathfrak{H}} \xi_{j} \overline{\xi_{k}}
$$


By Lemma 4.6 the form (4.57) has at most $\widetilde{\kappa}-\kappa$ and for some choice of $z_{j}, g_{j}$, $j=1, \ldots, n$, exactly $\tilde{\kappa}-\kappa$ negative squares.

In view of Lemma 3.2 in [16] this implies that the operator $\Phi_{1}(z)$ is invertible for all $z \in \rho\left(\widetilde{V}^{-1}\right) \cap \mathbb{D}$ except $\widetilde{\kappa}-\kappa$ points and

$$
\varepsilon(z)=\Phi_{2}(z) \Phi_{1}(z)^{-1} \in \mathcal{S}_{\widetilde{\kappa}-\kappa}\left(\mathfrak{L}_{1}, \mathfrak{L}_{2}\right) .
$$

In view of (4.58) the formula (4.54) can be rewritten as (4.34).

Remark 4.9 (1) For a standard isometric operator in a Pontryagin (resp. Kreĭn) space similar formulas for generalized coresolvents were found in [38-40] (resp. [28]). For the case of a nonstandard isometric operator in a Pontryagin space see [44]. An elegant proof of the formula for generalized resolvents of a nonstandard Pontryagin space symmetric operator with deficiency index $(1,1)$ given by $\mathrm{H}$. de Snoo was presented in [34]. In [5] a description of regular generalized resolvents of a nonstandard Pontryagin space isometric operator was given by the method of boundary triples. For a Hilbert space isometric operator this method was developed earlier in [41] and applied to the proof of Kreĭn type resolvent formulas (4.34), (4.36).

(2) The extension $V_{\varepsilon(z)}$ appearing in (4.52) is an analog of Shtraus extension, which was introduced in [47] for the case of a symmetric operator. In view of (4.53) the vector function $f_{z}=\mathbf{K}_{z} g$ can be treated as a solution of the following "abstract boundary value problem" with $z$-dependent boundary conditions

$$
\widehat{f_{z}}:=\left(\begin{array}{c}
z f_{z} \\
f_{z}-g
\end{array}\right) \in V^{-[*]}, \quad \Gamma_{2} \widehat{f_{z}}=\varepsilon(z) \Gamma_{1} \widehat{f_{z}} .
$$

(3) In abstract interpolation problem considered in [36] the crucial role was played by the Arov-Grossman formula for scattering matrices of unitary extensions of isometric operators, [3]. In [6] the formula for generalized coresolvents was applied to the description of scattering matrices of unitary extensions of Pontryagin space isometric operators which, in turn, was used in [7] for parametrization of solutions of an indefinite abstract interpolation problem, see also [15]. The present version of formula (4.34) will allow to consider $k$-regular indefinite interpolation problems with the growth of index $\kappa$.

Funding Open Access funding enabled and organized by Projekt DEAL.

Data Availability Data sharing not applicable to this article as no datasets were generated or analysed during the current study.

Open Access This article is licensed under a Creative Commons Attribution 4.0 International License, which permits use, sharing, adaptation, distribution and reproduction in any medium or format, as long as you give appropriate credit to the original author(s) and the source, provide a link to the Creative Commons licence, and indicate if changes were made. The images or other third party material in this article are included in the article's Creative Commons licence, unless indicated otherwise in a credit line to the material. If material is not included in the article's Creative Commons licence and your intended use is not permitted by statutory regulation or exceeds the permitted use, you will need to obtain permission directly from the copyright holder. To view a copy of this licence, visit http://creativecommons.org/licenses/by/4.0/. 


\section{References}

1. Alpay, D., Dijksma, A., Rovnyak, J., de Snoo, H.S.V.: Schur functions, operator colligations, and reproducing kernel Pontryagin spaces. Operational Theory: Advances and Applications. Birkhäuser, Basel (1997)

2. Arens, R.: Operational calculus of linear relations. Pac. J. Math. 11, 9-23 (1961)

3. Arov, D.Z., Grossman, L.Z.: The scattering matrices in the theory of the extensions of isometric operators. DAN USSR 33(4), 17-20 (1983)

4. Azizov, T.Ya., Iokhvidov, I.S.: Linear operators in spaces with indefinite metric. Wiley, New York (1989)

5. Baidiuk, D.V.: On boundary triplets and generalized resolvents of an isometric operators in a Pontryagin space. Ukr. Math. Bull. 10, 176-200 (2013)

6. Baidiuk, D.V.: Description of scattering matrices of unitary extensions of isometric operators in Pontryagin space. Math. Notes 94(6), 940-943 (2013)

7. Baidiuk, D.: Abstract interpolation problem in generalized Schur classes. arXiv:1403.4038 [math.FA] (2014)

8. Behrndt, J., Derkach, V., Hassi, S., de Snoo, H.S.V.: A realization theorem for generalized Nevanlinna families. Oper. Matrices 5(4), 679-706 (2011)

9. Bennewitz, Ch.: Symmetric relations on a Hilbert space. Lect. Notes Math. 280, 212-218 (1972)

10. Bernstein, D.: Matrix Mathematics. Princeton University Press, Princeton (2009)

11. Bognár, J.: Indefinite Inner Product Space. Springer, Berlin (1974)

12. Brodskii, M.S.: Unitary operator colligations and their characteristic functions. Uspekhi Mat. Nauk 33(4), 141-168 (1978)

13. Calkin, J.W.: Abstract symmetric boundary conditions. Trans. Am. Math. Soc. 45, 369-442 (1939)

14. Derkach, V.: On generalized resolvents of Hermitian relations in Kreŭn spaces. J. Math. Sci. 97(5), 4420-4460 (1999)

15. Derkach, V.A.: On indefinite abstract interpolation problem. Methods Funct. Anal. Topol. 7(4), 87-100 (2001)

16. Derkach, V., Dym, H.: On linear fractional transformations associated with generalized $J$-inner matrix functions. Integ. Eq. Oper. Theory 65, 1-50 (2009)

17. Derkach, V., Dym, H.: Rigged de Branges-Pontryagin spaces and their application to extensions and embedding. J. Funct. Anal. 277(1), 31-110 (2019)

18. Derkach, V.A., Hassi, S., Malamud, M.M., de Snoo, H.S.V.: Generalized resolvents of symmetric operators and admissibility. Methods Funct. Anal. Topol. 6(3), 24-55 (2000)

19. Derkach, V.A., Hassi, S., Malamud, M.M., de Snoo, H.S.V.: Boundary relations and Weyl families. Trans. Am. Math. Soc. 358, 5351-5400 (2006)

20. Derkach, V.A., Hassi, S., Malamud, M.M., de Snoo, H.S.V.: Boundary relations and generalized resolvents of symmetric operators. Russ. J. Math. Phys. 16(1), 17-60 (2009)

21. Derkach, V.A., Hassi, S., Malamud, M.M., de Snoo, H.S.V.: Boundary triples and Weyl functions. In: Recent developments, London Mathematical Society Lecture Notes 404, pp. 161-220 (2012)

22. Derkach, V.A., Hassi, S., Malamud, M.M.: Generalized boundary triples, Weyl functions and inverse problems. arXiv:1706.07948 104 p (2017)

23. Derkach, V.A., Hassi, S., Malamud, M.M.: Generalized boundary triples, I. Some classes of isometric and unitary boundary pairs and realization problems for subclasses of Nevanlinna functions. Math. Nachr. 293(7), 1278-1327 (2020)

24. Derkach, V.A., Hassi, S., Malamud, M.M.: Generalized boundary triples, II. Some applications of generalized boundary triples and form domain invariant Nevanlinna functions (submitted for publication)

25. Derkach, V.A., Malamud, M.M.: On the Weyl function and Hermite operators with lacunae. Dokl. Akad. Nauk SSSR 293(5), 1041-1046 (1987)

26. Derkach, V.A., Malamud, M.M.: The extension theory of hermitian operators and the moment problem. J. Math. Sci. 73, 141-242 (1995)

27. Derkach, V.A., Malamud, M.M.: Extension theory of symmetric operators and boundary value problems. Proc. Inst. Math. NAS Ukraine Kiev 104, 141-242 (2017)

28. Dijksma, A., Langer, H., de Snoo, H.S.V.: Generalized coresolvents of standard isometric relations and generalized resolvent of standard symmetric relations in Krĕn space. Operator theory: advances and applicatios, pp. 261-274. Birkhäuser Verlag, Basel (1990) 
29. Hassi, S., Sandovici, A., de Snoo, H.S.V., Winkler, H.: A general factorization approach to the extension theory of nonnegative operators and relations. J. Oper. Theory 58(2), 351-386 (2007)

30. Iokhvidov, I.S., Kreĭn, M.G.: The spectral theory of operators in spaces with indefinite metric, I. Trudy Mosk. Mat. Obshch. 5, 367-432 (1956)

31. Iohvidov, I.S., Krein, M.G., Langer, H.: Introduction to the spectral theory of operators in spaces with an indefinite metric, Mathematical Research, 9. Akademie, Berlin (1982)

32. Gohberg, I., Kre ̌n, M.G.: The basic propositions on defect numbers, root numbers and indices of linear operators, Uspekhi Mat. Nauk., 12, 43-118 (1957). (Russian) [English translation: Transl. Am. Math. Soc., 13(2), 185-264 (1960)]

33. Gorbachuk, V.I., Gorbachuk, M.L.: Boundary problems for differential operator equations. In: Naukova Dumka, Kiev (1984) (English transl.) Mathematics and its Applications, 48. Kluwer Academic Publishers Group, Dordrecht (1991)

34. Kaltenbäck, M., Woracek, H.: Generalized resolvent matrices and spaces of analytic functions. Integral Equ. Oper. Theory 32(3), 282-318 (1998)

35. Kochubei, A.N.: On extensions of symmetric operators and symmetric binary relations. Matem. Zametki 17(1), 41-48 (1975)

36. Katsnel'son, V.E., Kheifets, A.Ya., Yuditskii, P.M.: An abstract interpolation problem and the theory of the extensions of isometric operators. Operators in Functional Spaces and Problems in Function Theory (Russian), pp. 83-96. Kiev, Naukove Dumka (1987)

37. Kreĭn, M.G., Langer, H.: On defect subspaces and generalized resolvents of Hermitian operator in Pontryagin space, Funkts. Anal. i Prilozhen., 5(2), 59-71, (1971); ibid. 5(3), 54-69 (1971),(Russian) [English translation: Funct. Anal. Appl., 5 (1971), 136-146; ibid. 5, 217-228 (1971)]

38. Kreı̆, M.G., Langer, H.: Über die verallgemeinerten Resolventen und die characteristische Function eines isometrischen Operators im Raume $\Pi_{\kappa}$. Hilbert space Operators and Operator Algebras. Proc. Intern. Conf., Tihany, (1970). Colloq. Math. Soc. Janos Bolyai, North-Holland, Amsterdam, 5, 353399 (1972)

39. Langer, H.: The generalized coresolvents of a $\pi$-isometric operator with unequal defect numbers. Funkcional. Anal. i Prilozen. 5(4), 73-75 (1971). (Russian)

40. Langer, H., Sorjonen, P.: Verallgemeinerte Resolventen hermitescher und isometrischer Operatoren im Pontrjaginraum. Ann. Acad. Sci. Fenn. Ser. A. I., (561), 1-45 (1974)

41. Malamud, M.M., Mogilevskii, V.I.: The generalized resolvents of an isometric operator. Mat. Zametki 73(3), 460-466 (2003)

42. Malamud, M.M., Mogilevskii, V.I.: The resolvent matrices and the spectral functions of an isometric operator. Docl. Akad. Nauk 395(1), 11-17 (2004)

43. Nitz, O.: Generalized resolvents of isometric linear relations in Pontryagin space, 1: Foundations. Oper. Theory Adv. Appl. 118, 303-319 (2000)

44. Nitz, O.: Generalized resolvents of isometric linear relations in Pontryagin space, 2: Krein-Langer formula. Methods Funct. Anal. Topol. 6(3), 72-96 (2000)

45. Shmul'yan, Yu.L.: Theory of linear relations and spaces with indefinite metric. Funkts. Anal. Pril. 10(1), 67-72 (1976)

46. Sorjonen, P.: Generalized resolvents of an isometric operator in a Pontrjagin space. Z. Anal. Anwendungen 4(6), 543-555 (1985)

47. Štraus, A.V.: Extensions and generalized resolvents of a symmetric operator which is not densely defined, Izv. Akad. Nauk. SSSR, Ser. Mat., 34, 175-202 (1970). (Russian) [English translation: Math. USSR-Izvestija, 4, 179-208 (1970)]

Publisher's Note Springer Nature remains neutral with regard to jurisdictional claims in published maps and institutional affiliations. 